\title{
Conference on Emergency Medicine, Oxford, 27-30 September 1998 (Hosted by the Faculty of Accident and Emergency Medicine in association with the American Society of Academic Emergency Medicine)
}

EMRS Prize Session, Monday 28 September

The association between seniority of accident and emergency doctor and outcome following trauma

J P Wyatt, J Henry, D Beard

Accident and Emergency Department, Royal Infirmary of Edinburgh, Lauriston Place, Edinburgh $E H 3$ 9YW

Aim-To investigate the hypothesis that the seniority of accident and emergency (A\&E) doctors treating patients after trauma is important in determining their outcome.

Methods-10968 patients presenting to the A\&E department after trauma in four Scottish teaching hospitals during the five years 1992-96 were studied prospectively. Criteria for inclusion were those previously used by the Major Trauma Outcome Study. Data collected included age, mechanism of injury, seniority of $A \& E$ doctor, physiological variables (respiratory rate, Glasgow coma score, and systolic blood pressure) at presentation, anatomical injuries and outcome (measured in terms of survival or death). Revised trauma scores and injury severity scores were derived, allowing probabilities of survival to be calculated according to TRISS methodology. The actual survival of patients treated after trauma was compared with that predicted according to TRISS analysis using UK coefficients.

Results-Physiological data were complete for 10968 patients, a data capture rate of $94 \%$. All those who died as a result of their injuries were included. The 1208 patients treated by a consultant $(W$ statistic $=3.15 ; 95 \%$ confidence interval 1.82 to 4.47 ) had a significantly better outcome $(p<0.05)$ than the 9195 patients treated by junior doctors (W statistic $=0.93 ; 95 \%$ confidence interval 0.59 to 1.27). Analysis of outcome according to the grade of junior $A \& E$ doctor (senior house officer, registrar, senior registrar) showed a stepwise improvement in outcome with seniority.

Conclusion-This study provides evidence that an improved outcome after trauma is associated with increased experience and seniority of $A \& E$ doctor. These results support calls for A\&E consultants to be increasingly involved in the management of patients with major trauma. Such increased involvement would require a further increase in the number of A\&E consultants.

The cardiovascular effects of the right and left lateral recovery positions

M J Stuart, S Derbyshire, K Mackway-Jones 16 St Wilfred's Drive, Healey, Rochdale OL12 $6 D B$

Background-After successful resuscitation patients are placed in the recovery position.
Current resuscitation guidelines do not distinguish between left and right lateral positions. Purpose-This study was designed to investigate the cardiovascular consequences of the left and right lateral recovery positions. Materials and methods-Twenty healthy volunteers (10 male, 10 female) were recruited into the study. Stroke distance, which is an assessment of left ventricular stroke volume, was measured non-invasively using a $2 \mathrm{MHz}$ ultrasound probe (Deltex Medical Ltd), placed in the suprasternal notch employing the Doppler principle with the volunteers in the supine, left lateral , and right lateral recovery positions. Stroke distance in the left and right positions was expressed as a percentage of that in the supine position. Measurements in the left lateral position revealed a stroke distance of $88.6 \%( \pm 0.099)$ of supine values. Measurements in the right lateral position showed a mean distance of $97.6 \%( \pm 0.141)$ of that found in the supine position. Statistical analysis, using a two sample Student's test assuming unequal variance, showed a significant difference between supine and left lateral positions $(p<0.0001)$, and a significant difference between left and right lateral position $(p=0.014)$. There was no significant difference between supine and right lateral positions.

A similar experiment was carried out using thoracic bioimpedance as a measure of cardiac output. The results of this study will be available for presentation.

Conclusions-This study has shown that there is a significant fall in stroke distance (a proxy for cardiac output) in the left lateral position compared with the supine and right lateral positions. If a patient requires to be in a lateral position to protect their airway after resuscitation, then the right lateral position should be used as it has no detrimental effect on cardiac output.

Magnesium for refractory ventricular fibrillation-a randomised study to investigate its role

T B Hassan ${ }^{\star}$, D B Barnett ${ }^{\star \star}$

${ }^{\star}$ Department of Accident and Emergency Medicine, and ${ }^{*}$ Department of Pharmacology and Therapeutics, Leicester Royal Infirmary NHS Trust, Leicester LE1 5WW

Background-Ventricular fibrillation remains the commonest and most salvageable rhythm in patients suffering a cardiopulmonary arrest. However, outcome remains poor if there is no response to initial defibrillation. Some evidence suggests that intravenous magnesium may prove to be a novel antiarrhythmic agent in such circumstances.

Study hypothesis - Intravenous magnesium sulphate given early in the resuscitation phase for patients in refractory ventricular fibrillation (ventricular fibrillation after $3 \mathrm{DC}$ shocks) or recurring ventricular fibrillation will signifi- cantly improve their outcome to discharge from hospital alive.

Design-A randomised, double blind, placebo controlled trial. Predefined primary and secondary endpoints were return of spontaneous circulation (ROSC) at the scene or in the accident and emergency (A\&E) department and discharge from hospital alive respectively. To identify a $15 \%$ improvement in the primary endpoint powered at $84 \%$ with a two tailed significance level of 0.05 required recruitment of 50 patients per arm.

Setting, participants, and intervention-Patients treated in the pre-hospital phase by the county emergency medical service and/or in the $A \& E$ department. One hundred and five patients with refractory ventricular fibrillation were recruited to the study over a 15 month period and randomised to receive either 2-4 $\mathrm{g}$ of magnesium sulphate or placebo intravenously.

Results-Fifty two patients received magnesium treatment and 53 received placebo. The two groups were matched for most parameters including age, sex, response time for arrival at scene, and airway interventions. There were no significant differences between magnesium and placebo for ROSC at the scene or in the A\&E department (9 patients (17\%) $v 7$ patients $(13 \%)$ ), Pearson's $\chi^{2}$ test, $p=0.56$, and alive to discharge from hospital (2 (4\%) $v$ $1(2 \%)) \chi^{2}$ test, $\mathrm{p}=0.55$. Using multivariate logistic regression the odds ratios $(95 \%$ confidence intervals) for ROSC and discharge alive from hospital in patients treated with magnesium compared with placebo were $1.81(0.51$ to 6.37$)$ and 5.23 (0.31 to 89.1$)$ respectively. Conclusion-Intravenous magnesium when given empirically early on in the pre-hospital resuscitation phase may have a part to play in treating refractory ventricular fibrillation.

Improving doctors' training. A control trial

Simon J Ward

40 Rodwell Road, London SE22 9LE

Introduction-An unresolved conflict exists between the reduction in the duration of junior hospital doctors' training and a concurrent increase in the intensity and demand of their service workload. A solution to this problem is to enhance the quality of existing training, and in particular "on-the-job training" (OJT). This research concentrates on one area of OJT and produces evidence that trainees can become more skilled at learning for themselves and more responsible for their learning. Methods-Nineteen trainees participated in the intervention over four months at a district general hospital and were compared with a matched control group $(n=13)$ at the same hospital. The intervention focused on learning skills from the OJT guides. These encouraged and empowered trainees to learn for themselves and hence gain more from their 
training. Multiple cross validating evaluation methodologies were used. These included questionnaires, self completion evaluations, observational and qualitative evidence. Participation was over $90 \%$.

Results-Statistically significant ( $1<0.05)$ changes were found for the intervention group in the learning outcomes of taking control of learning; making full use of learning opportunities during service work; eliciting feedback; coping with the job; self esteem; and confidence to assume clinical responsibility. The control group showed trends toward being significantly worse at taking control of their learning $(p=0.056)$. Qualitative findings were in concordance with and finessed the quantitative findings. These former findings showed that an individual approach is necessary where the particular needs of each trainee are taken intoaccount. This involved an integrated and flexible approach to training and service, while using a partnership between trainer and trainee.

Conclusions - This research found evidence in support of the hypothesis that trainees can enhance their training capacity, and hence improve the quality of their training, using OJT skills. Self esteem and confidence of trainees were also improved.

A randomised study to evaluate the usefulness of a single occipitomental $x$ ray in suspected mid-facial injury: the SIOMEX Study

D Vickery*, T B Hassan ${ }^{\star}$, S K Kirkpatrick ${ }^{\star}$, E Brazil ${ }^{\star}$, G Tudor ${ }^{\star \star}$, D Finlay ${ }^{\star \star}, G$ G Bodiwala $\star$

${ }^{\star}$ Department of Accident and Emergency Medicine, and ${ }^{\star *}$ Department of Radiology, Leicester Royal Infirmary NHS Trust, Leicester LE1 $5 W W$

Background-Patients with bony maxillofacial injury commonly present to accident and emergency (A\&E) departments in the UK. Correct diagnosis relies upon three $x$ ray views of the mid-face as a standard, although evidence suggests that a single $15^{\circ}$ occipitomental (OM-15) $x$ ray may be adequate. Significant benefits would be incurred both in terms of less radiation exposure to the head as well as financial savings for an institution.

Study hypothesis-Correct interpretation by $\mathrm{A} \& \mathrm{E}$ staff of a single OM-15 view is equivalent to that of a standard three $x$ ray set of views in the assessment of bony mid-facial injury.

Design-A prospective randomised study. The "gold standard" was provided by a senior radiologist interpreting the standard three $x$ ray set of views.

Setting, participants, and randomisation-Adult patients with suspected mid-facial injury assessed and treated in a university teaching hospital $A \& E$ department were eligible for entry into the study. On alternate days medical staff in the A\&E department were asked to interpret either a single OM-15 view (with the other two in a sealed envelope) or all three $x$ ray views and record their findings on a proforma.

Results-During the nine month study period, A\&E doctors interpreted a single OM-15 view in 160 patients and the standard three view set in 139 patients. A total of 53 patients were identified by the gold standard as having a definite or possible mid-face fracture (study prevalence $=18 \%$ ). Study results are shown in table 1 .
Table 1

\begin{tabular}{lll}
\hline & OM-15 view & $3 \times$ rays \\
\hline Sensitivity (\%) & 56 & 65 \\
Specificity (\%) & 90 & 91 \\
Positive predictive value (\%) & 54 & 63 \\
Negative predictive value (\%) & 91 & 92 \\
\hline
\end{tabular}

Conclusions - An OM-15 view is equivalent to a standard three view set where there is no bony injury but interpretation is worse as compared with the three views when injury is confirmed. Junior A\&E staff frequently miss radiological abnormalities when interpreting facial $x$ rays.

\section{Adverse circadian variation in survival in} the older trauma victim

C G McMahon, S Hollis, D W Yates, M A Woodford

University Department of Emergency Medicine, Hope Hospital, Salford M6 $8 \mathrm{HD}$

Objective-To examine the influence of hour of day on survival after multiple trauma.

Design-Observational study.

Methods-In order to examine the effect of hour of day on survival after injury an analysis of the UK Trauma Audit and Research Network database was carried out. The study group consisted of 57333 patients admitted to over 140 hospitals throughout the UK between 1990 and 1996. Data was collected on both elements of the process of care and details of the mechanism and severity of injury.

The 1990 revision of the abbreviated injury scale was used to calculate overall injury severity score (ISS). We compared mortality for three different injury severity groups: mild (ISS 1-8), moderate (ISS 9-15), and severe (ISS $16+$ ) over the 24 hour period. The effect of age greater than or less than $\mathbf{5 5}$ years was also analysed. Mortality rates on weekdays were compared with weekend days. The percentage of these patients seen by a senior doctor within one hour of arrival was determined.

Results-Logistic regression analysis accounting for age and injury severity demonstrated that hour of day has a significant effect on trauma outcome $(p=0.0005)$. Weekdays were not significantly different to weekend days $(p=0.19)$. This adverse effect was only seen in patients over the age of 55 years with mild or moderate injury presenting between the hours of $02.00-06.00$. Mortality increased from $2.95 \%$ to $8.3 \%$ and $5.5 \%$ to $12.8 \%$ in the mild and moderately injured groups respectively in patients over the age of 55 years. There was no significant diurnal variation in outcome seen in the younger patients.

Analysis of the percentage of patients seen within one hour of arrival demonstrated that patients over the age of 55 years are less likely to be seen by a senior doctor at any time of the day. This finding was even more marked at their highest risk time when less than $6 \%$ of the group was seen early by a senior compared with $25 \%$ of patients with similar injury severity who are less than 55 years.

Conclusion-Hour of day has an unexpected effect on survival in patients over the age of $\mathbf{5 5}$ years presenting with mild or moderate injury. Mortality increases twofold between the hours of $02.00-06.00$ in this group.
Free Paper Session I: Imaging, Tuesday

\section{September}

Evaluation of a protocol including dynamic cervical screening for spinal clearance in the unconscious trauma patient R A Brooks, K M Willett

Department of Trauma, fohn Radcliffe Hospital, Headington, Oxford

Purpose-To study the means by which spinal immobilisation may be safely discontinued in the unconscious multiply injured patient, with particular reference to the use of dynamic screening of the cervical spine under fluoroscopic control.

Methods and results-Assessment of the spine in the unconscious multiply injured patient at our trauma unit since April 1994 includes plain anteroposterior and lateral radiographs of the whole length of the spine, computed tomography of $\mathrm{C} 1$ and $\mathrm{C} 2$ and dynamic flexion and extension screening of the cervical spine under fluoroscopic control.

The results of this policy over a 3.5 year period in 210 patients were reviewed. Forty one patients had a spinal fracture. Nineteen patients had a fracture at more than one level and these were non-contiguous in nine patients. The diagnosis of spinal fracture was made before extubation in all but two patients who died before investigations were completed. Three patients developed occipital pressure sores relating to prolonged use of a rigid cervical collar. Seventy eight patients underwent dynamic screening of the cervical spine. Five of these patients had a cervical fracture or instability. Three had been identified on plain radiography but screening was used to determine stability. In another patient, a stable spinous process fracture became visible on screening. The fifth patient demonstrated minor changes on plain radiography but gross instability on dynamic screening. There were no neurological sequelae in these or any other patients. Collar removal was therefore possible in all but one patient a median three days before extubation and one day after admission to the intensive care unit. Conclusion - In our limited study a full length spinal radiography protocol with computed tomography of $\mathrm{C} 1$ and $\mathrm{C} 2$ and dynamic screening of the cervical spine allows early and safe discontinuation of spinal precautions to facilitate nursing care and minimise complications in the unconscious patient.

Verification of endotracheal intubation using ultrasound

Michael J Drescher, Frederick V Conard, Neil S Schamban

Department of Traumatology and Emergency Medicine, University of Connecticut School of Medicine, USA

Objective-Verification of endotracheal tube placement is a vital step in emergent airway management. This study was undertaken to test the hypothesis that ultrasound is reliable in the detection of oesophageal intubations. To date there are no studies evaluating ultrasound for this purpose.

Methods-The study was done in an urban level I teaching hospital. We conducted a nonblinded prospective observational study of three groups of patients using B-mode ultrasound of the trachea and of the oesophagus. Institutional review board approval was obtained. Evaluation was performed by an emergency physician and a radiologist using an Acuson XP-10 7.5 megahertz linear array 
transducer. Patients with previously placed endotracheal tubes were scanned in a medical intensive care unit $(n=8)$. A second group of newly deceased patients were purposefully intubated in the oesophagus postmortem $(n=4)$. The oesophageal intubations were observed in real time using ultrasound. A third group of healthy volunteers was evaluated as controls $(n=4)$.

Results-Preliminary results show that ultrasound can distinguish between oesophageal and endotracheal intubation in real time. An oesophageal tube is well visualised in the paratracheal position, dynamically, as documented on video tape at the time of intubation, and statically thereafter. In addition, there is a consistent periodic resonating artifact found in the non-intubated trachea which is not present in the intubated trachea. Conclusions-Results of this ongoing study show that ultrasound can verify endotracheal intubation and detect oesophageal intubation in real time. These findings will need to be validated in greater numbers of patients in a clinical setting. However, they suggest that ultrasound may add to the armamentarium of emergency physicians in airway management.

Derivation of decisions guidelines for emergency department utilisation of non-contrast head computed tomography in HIV infected patients

Richard Rothman, Justin McArthur, Taras Danyluk, Farzaneh Tabrizi, Norman Beauchamp, Gabor D Kelen

fohns Hopkins University, Department of Emergency Medicine, Marburg B186, $600 \mathrm{~N}$ Wolfe St, Baltimore, MD 21287-2080, USA

Objective-To describe clinical criteria predictive of new focal findings on head computed tomography in HIV infected patients with neurological complaints.

Methods-Prospective cohort analysis with consecutive enrollment of all HIV infected patents presenting to a university based emergency department over an 11 month period. HIV infected patients with any neurologica complaint were evaluated using a standardised questionnaire, designed to detect new or changed neurological signs or symptoms. Al head scans were done while the patient was in the emergency department. The association between new neurological findings and new focal lesions on head computed tomography were analysed using one way analysis of variance.

Results - Of 110 patients meeting inclusion, 27 (24\%) had focal lesions on computed tomography of the head. Of these, 19 were new focal lesions and eight demonstrated a mass effect. Clinical parameters strongly associated with new findings on head computed tomography were (a) seizure, new or changed in pattern; (b) focal motor deficits; (c) change in mental status; and (d) headache, new or different in quality. Application of these four parameters as a screening tool would have identified $100 \%$ of the patients with new intracranial lesions, and resulted in a $25 \%$ reduction in emergency department utilisation of head computed tomography. The aetiology most commonly responsible for new focal findings among patients with CD4 less than 200 was infection (65\%), versus ischaemic lesions or encephalopathy $(83 \%)$ in those with CD4 counts greater than 200. Al patients with new focal findings required intervention and hospitalisation, with $85 \%$ requiring life saving intervention within the first 24 hours.
Conclusions-Specific clinical signs and symptoms are associated with the presence of new intracranial lesions in HIV infected patients who present to the emergency department with new neurological complaints. Validation and widespread application of these guidelines could lead to more selective and cost effective use of computed tomography of the head in the HIV infected population in the emergency department.

Comparison of non-contrast spiral computed tomography against intravenous pyelography in the evaluation of acute flank pain

Scott K Rineer, Oren F Miller, Scott R Reichard, Murray S Donovan, Ian R Graham, Walter B Goff, Robert G Buckley, Christopher J Kane

Naval Medical Center, San Diego, CA, USA

Objectives-To determine the value of unenhanced spiral computed tomography (NCCT) compared with intravenous pyelography (IVP) in the evaluation of acute flank pain.

Methods-Between 11 May 1997 and 29 September 1997 each patient presenting through the emergency department or urology clinic at a large tertiary referral military hospital was offered enrollment in this study. An observational, prospective evaluation of 106 consenting adult patients with acute flank pain received imaging via NCCT followed by IVP. Each imaging modality was read by a single radiologist blinded to both the opposing imaging technique and clinical history. Subsequent follow up was scheduled within 72 hours in the urology clinic. Sensitivity, specificity, and positive and negative predictive values were determined for NCCT and IVP. Inclusion criteria were age over 18 and acute flank pain, with or without microhaematuria Patients were excluded if they presented with a creatinine over $2.0 \mathrm{mg} / \mathrm{dl}$, age less than 18 years, pregnancy, or had an allergy to radiocontrast or iodine. Research was approved by our institutional review board.

Results-Of 106 patients who presented with acute flank pain the diagnosis of ureterolithiasis was made in 75 patients $(71 \%)$. Of the 31 patients without ureterolithiasis, the NCCT was negative in all cases. IVP was negative in 29 of the 31 cases. NCCT was $96 \%$ sensitive and $100 \%$ specific $(p<0.001)$. IVP was $87 \%$ sensitive and $94 \%$ specific $(p<0.001)$. When compared with IVP, NCCT was significantly better able to predict the presence of urolithiasis $(\mathrm{p}=0.015)$.

Conclusion-NCCT accurately diagnoses ureterolithiasis in patients presenting with acute flank pain. NCCT is significantly better than IVP in determining the presence of urolithiasis.

The use of electron base computed tomography in the evaluation of chest pain patients in the emergency department

Dennis A Laudon, Larry F Vukov, Jerome F Breen, John A Rumberger, Peter C Wollan, Patrick F Sheedy II

Mayo Clinic and Foundation, 200 First Street SW, Rochester, MN 55905, USA

Objective-The detection of coronary artery calcification by electron beam computed tomography (EBCT) is highly predictive of the presence of coronary disease and the absence of coronary calcification is highly specific for the absence of obstructive coronary artery disease. This study was designed to see if the
EBCT could be used in the emergency department as a triage tool in patients who present with anginal-like chest pain, with no known history of coronary disease and with normal initial electrocardiograms (ECGs) and cardiac enzymes.

Methods-A prospective observational study of 105 patients who presented to the emergency department of a large tertiary care hospital, between December 1995 and October 1997. Random sampling was done of women $40-65$ years of age and men $30-55$ years of age who presented with anginal-like chest pain requiring admission. All patients received an EBCT of their coronary arteries along with other cardiac testing as determined by a staff physician.

Result - Of the 105 patients, 100 had other cardiac testing done during their hospitalisation. Evaluations included treadmill exercise testing in 58, coronary angiograms in 25 , radionuclide stress testing in 19 , and stress echocardiograms in 11. Fifty nine patients $(56 \%)$ had both negative cardiac evaluations and negative EBCTs. Fourteen (13\%) had positive EBCTs and positive cardiac testing. Thirty two $(30 \%)$ had positive EBCTs and negative cardiac testing. Sensitivity for the EBCT in this study was $100 \%$ (95\% confidence interval of $77 \%$ to $100 \%$ ) with a negative predictive value of $100 \%$ ( $94 \%$ to $100 \%)$. Specificity was $63 \%$ (54\% to $75 \%$ ).

Conclusions-EBCT is an efficient screening tool for patients presenting to the emergency department with anginal-like chest pain who have normal enzymes, indeterminate ECGs, and no history of coronary artery disease. Our study suggests that those patients with normal initial cardiac enzymes and electrocardiograms and a negative EBCT can be safely dismissed from the emergency department without further testing or observation.

Free Paper Session II: Trauma 1, Tuesday 29 September

Evaluation of neurosurgical referral letter

J Keany, N Fitzpatrick, D Beard, D Ritchie, L Dunn

Accident and Emergency Department, Victoria Infirmary, Langside, Glasgow

Background-A standardised regional neurosurgical referral letter (NRL) was developed by the Scottish Trauma Audit Group (STAG) in conjunction with a multispecialty group in south west Scotland. The purpose of the letter was to improve communication when transferring patients with head injuries, and to act as an aide-memoire in the assessment of the patient.

Objectives - This study aimed to evaluate the use of the NRL by four criteria: compliance, completeness, comparison between NRL and standard transfer letters, and clinical relevance.

Methods-The Institute of Neurological Sciences serves a population 2.8 million in the south west of Scotland. Patients who were referred from accident and emergency (A\&E) departments during the period 1 September 1997 to 28 February 1998 were eligible for inclusion in this prospective audit. Compliance was measured by completion rate in the 10 STAG hospitals who use the NRL, completeness, that is the degree to which each NRL was completed, comparison with transfer information accompanying patients from non-STAG hospitals, and clinical 


\begin{tabular}{llllll}
\hline & GCS & Pupil size & Blood pressure & $\mathrm{O}_{2}$ sat & $\begin{array}{l}\text { Extracranial } \\
\text { injuries }\end{array}$ \\
\hline STAG with NRL & $74(92)$ & $73(91)$ & $67(84)$ & $73(91)$ & $69(86)$ \\
STAG no NRL & $5(42)$ & $5(42)$ & 0 & $3(25)$ & $5(42)$ \\
Non-STAG hospitals & $33(87)$ & $14(37)$ & $1(3)$ & $18(47)$ & $21(55)$ \\
\hline
\end{tabular}

Values are number $(\%)$. GCS=Glasgow coma score; $\mathrm{O}_{2}$ sat=oxygen saturation.

relevance as determined by postal questionnaires sent to $A \& E$ doctors and neurosurgeons. Identification and weighting of key variables was undertaken by consultant neurosurgeons.

Results-Of the 92 patients transferred from STAG A\&E departments, $80(87 \%)$ had an NRI completed; 38 patients were transferred from non-STAG hospitals. The completeness rate for the key variables identified by the neurosurgeons are listed in table 2 .

The NRL was thought to be clinically relevant by $94 \%(67 / 71)$ of A\&E doctors surveyed who replied end by $93 \%(14 / 15)$ of neurosurgeons.

Conclusions-There was widespread acceptance of the NRL in south west Scotland. The completeness rate of key variables was significantly higher when the NRL was used. The majority of clinicians involved in the transfer process considered the NRL to be clinically relevant. We conclude that the NRL facilitates the provision of concise, relevant, clinical information fundamental to good communication.

\section{Pre-existing cardiovascular disease in} UK trauma patients

G Brown, P Driscoll, P Grant, S Hollis, T Wardle, $M$ Woodford, D W Yates

clo UK Trauma and Research Network, Clinical Science Building, Hope Hospital, Salford

Introduction-Pre-existing medical conditions (PEMC) have been shown to significantly increase mortality after injury. A previous UK study from one centre identified cardiovascular disease as the commonest PEMC in trauma patients. This study also suggested that cardiovascular disease is an independent predictor of mortality after injury. However, it is not known if these results are common to other UK centres.

Aims - (1) To assess the prevalence of cardiovascular disease on the UK Trauma Audit and Research Network (UKTARN) database and (2) to calculate the effect of pre-existing cardiovascular disease on mortality in the UK trauma patient.

Methods-Cases from the UKTARN database (55 032 entries since 1992) were grouped into 12 bands based on their injury severity score (ISS 1-15, 16-75), revised trauma score (RTS 7.84 or $<7.84)$, and their age $(<46,46-65$ or $>65)$. Each band was divided into two arms based on their coding for disease status (cardiovascular disease or no PEMC) and mortality frequencies calculated.

Logistic regression analysis was used on the entire data set (including "missing" data and other PEMCs) to assess whether cardiovascular disease is a significant independent predictor of mortality.

Results-Pre-existing cardiovascular disease is the commonest recorded PEMC on the UKTARN database. Frequency of death is greater in the group coded for pre-existing cardiovascular disease in all bands, except those containing only a few patients. Excluding these bands, the increase in mortality frequency varies from 1.09 (ISS 16-75, RTS $<7.84$, age $>65$ group) to 12.00 (ISS $1-15$, mortality is a national feature.

${ }^{\star}$ Accident and Emergency Medicine Academic
RTS 7.84, age $>65$ group) times greater in the cardiovascular disease group. Logistic regression analysis of the entire data set indicates that after controlling for injury severity and age, the risk of death is doubled in the group coded for cardiovascular disease compared with all other cases (odds ratio $=1.9711,95 \%$ confidence interval 1.68 to 2.31).

Conclusions-The results indicate that: (1) cardiovascular disease is the commonest recorded PEMC in the UK trauma patient and (2) the effect of cardiovascular disease on

Injury severity scores and prediction of post-traumatic complications

T H Rainer ${ }^{\star}$, R A Cocks ${ }^{\star}, M_{\text {Tam}}^{\star}, \mathrm{K} \mathrm{Law}^{\star}$, P Lam ${ }^{\star \star}, \mathrm{E}$ Wong ${ }^{\star \star}$ Unit, and ${ }^{\star}{ }^{\star}$ Centre for Clinical Trials and Epidemiological Research, Chinese University of Hong Kong and Prince of Wales Hospital, Shatin, Hong Kong

Background-A new injury severity score (NISS) has been proposed which accurately predicts post-traumatic mortality better than the injury severity score (ISS). However the performance of NISS has not been compared with ISS or the maximum abbreviated injury severity score (MAIS) as a predictor of post-traumatic pulmonary injury. This study compares the predictive value of NISS with ISS for mortality and lung injury.

Methods-Patients sustaining blunt trauma and triaged to the resuscitation room of the emergency department were included. Acute lung injury (ALI) and acute respiratory distress syndrome (ARDS) were defined according to recent American-European consensus statements. Fisher's exact tests and receiver operator curve characteristics (ROC) curves were used for analysis. Significance level was $\mathrm{p}<0.05$.

Results-96 subjects with a mean age of 37 years (SD 15, 79 males) and a median ISS of 12 (range 2-57) were included. Primary outcomes were ALI $(n=7)$, ARDS $(n=4)$, and total mortality $(n=13)$ within 28 days. Sensitivity, specificity, and ROC of injury scores in relation to outcomes are shown in table 3.

Conclusions-ISS is a better predictor than NISS and MAIS for post-traumatic lung injury but not for overall mortality.

Table 3 Performance of ISS to predict post-traumatic lung injury and mortality

\begin{tabular}{llllllll}
\hline Outcome & Injury score & Sensitivity & $95 \%$ CI & Specificity & $95 \%$ CI & Injury score & ROC area \\
\hline ALI & ISS $>27$ & 100 & $65.2,100$ & 86.5 & $70.5,99.3$ & ISS & 0.9518 \\
& NISS $>27$ & 100 & $65.2,100$ & 80.9 & $71.2,88.5$ & NISS & 0.9254 \\
\multirow{4}{*}{ ARDS } & MAIS $>3$ & 100 & $65.2,100$ & 76.4 & $66.2,84.8$ & MAIS & 0.9101 \\
& ISS $>27$ & 100 & $54.9,100$ & 84.6 & $75.5,91.3$ & ISS & 0.9077 \\
& NISS $>27$ & 100 & $54.9,100$ & 74.7 & $64.5,83.3$ & NISS & 0.8736 \\
Mortality & MAIS >3 & 100 & $54.9,100$ & 74.4 & $64.5,83.3$ & MAIS & 0.8747 \\
& ISS $>27$ & 76.9 & $46.2,95.0$ & 85.1 & $76.3,91.6$ & ISS & 0.8965 \\
& NISS $>27$ & 92.3 & $64.0,99.8$ & 76.6 & $66.7,84.7$ & NISS & 0.9272 \\
& MAIS $>3$ & 100 & $79.4,100$ & 24.5 & $16.2,34.4$ & MAIS & 0.9055 \\
\hline
\end{tabular}

$\mathrm{CI}=$ confidence interval.
Cyclic-AMP: the missing link in neutrophil mobilisation after trauma?

Robert A Cocks ${ }^{\star}$, Timothy H Rainer ${ }^{\star}$, Paula F Maycock ${ }^{\star \star}$, Tina Y F Chan ${ }^{\star}$, Nicole Y L $\mathrm{Lam}^{\star}$

*Accident and Emergency Medicine Academic Unit, Chinese University of Hong Kong, Hong Kong and ${ }^{*}$ North Western Injury Research Centre, Manchester

Introduction-Plasma from recently injured patients is capable of inducing a reduction in neutrophil adhesion behaviour, and this reduction can be partially blocked by propranolol. While adrenaline appears to play an important part in neutrophil mobilisation after trauma, a further unknown plasma factor is present. Cyclic-AMP (cAMP) is an intracellular messenger which mediates many of the effects of $\beta$ adrenergic stimulation of neutrophils, including granule stabilisation and reduction of adhesion molecule expression. It is detectable in plasma in normal subjects (range 16-25 pmol/ml) and is increased in the plasma during pregnancy, exercise, and starvation. This study investigates the levels of cAMP in the plasma in the first three hours after trauma.

Methods $-20 \mathrm{ml}$ samples of venous blood were drawn from 34 trauma patients within three hours of injury and divided between three sample tubes: EDTA for full blood count, cooled EDTA for cAMP levels, and cooled sodium heparin for catecholamines. The latter two tubes were immediately centrifuged at low temperatures and the supernatant plasma frozen at $-70^{\circ} \mathrm{C}$ pending analysis. Adrenaline was measured using HPLC and CAMP measured by an enzyme immunoassay technique.

Results - 34 patients were studied, seven of whom had sustained minor trauma (injury severity score (ISS) 1-8), 12 moderate trauma (ISS 9-15), and 15 major trauma (ISS >16). Median age was 39 years (range 16-77) and 30 patients were male. Plasma adrenaline levels were available for 28 of the patients.

Twenty six patients $(76 \%)$ had increased neutrophil counts (mean 11.3, range 0.4-25.3 $\left.\times 10^{9} / 1\right)$ and in all but three cases this increase was associated with a raised level of plasma adrenaline $(>0.8 \mathrm{nmol} / \mathrm{l})$. In two of these three patients, a raised level of plasma free cAMP was detected. Analysing the data from all patients, there were positive correlations between the plasma levels of cAMP and adrenaline $(p=0.014)$, adrenaline and neutrophil count $(p=0.0076)$, and cAMP

Conclusions-Plasma free cyclic AMP is raised in the majority of trauma patients and may play an important intracellular and extracellular part in neutrophil-endothelial cell interaction. and neutrophil count $(p=0.01)$. 
Myocardial contusion: does it warrant separate consideration during emergency department evaluation of blunt chest trauma?

E A Panacek, B Sherman, E L Priem

Emergency Medicine, UC Davis Medical Center, Sacramento, CA and Critical Care Medicine, Mount Sinai Hospital and University Hospitals, Cleveland, $\mathrm{OH}, \mathrm{USA}$

Though the inpatient evaluation of myocardial contusion has been well studied, emergency department predictors of clinically significant cardiac injury in patients with blunt chest trauma have not been well established. This study attempted to identify such respective predictors. The charts of all patients (187) admitted to two referral hospitals with a diagnosis of myocardial contusion during a five year period were reviewed. Patients were divided into two groups: group I (108) were those with no other injuries requiring admission. Group II (79) were those with additiona injuries requiring hospitalisation, separate from myocardial contusion. All patients received continuous electrocardiographic (ECG) monitoring for at least 24 hours. The mean age, gender, and mechanism of injury were similar in each group. Clavicle and rib fractures were the most common injury in group II $(52 \%)$, followed by extremity fractures $(20 \%)$. Arrhythmias were noted on initial emergency department evaluation in 11 patients from group II and one from group I. Five patients had $\geqslant 6$ PVCs/per min, three had SVT, three had less frequent PVCs, and one had transient A-fib. Six other patients from group II had bundle branch blocks and one from group I had first degree AV block. No patients developed additional arrhythmias requiring treatment that were not present on initial emergency department evaluation. Two patients in group I and seven in group II had elevated CPK-MB studies. None of these patients required specific treatment. Twenty three patients from group II had surgery requiring general anesthesia. None experienced cardiac complications. All study patients survived to hospital discharge.

Myocardial contusion is a diagnosis that has clinical significance in a small minority of blunt trauma patients. Its importance may be over emphasised. ECG abnormalities, when they occur, are apparent on admission Patients without significant injury or arrhythmias on initial presentation do not later develop significant rhythm complications from blunt myocardial trauma. We found no patients who required hospital admission only for myocardial contusion, separate from other significant injuries.

Free Paper Session III: Paediatrics/ Testing, Tuesday 29 September

\section{Defining criteria indicative of the need for hospitalisation and predictive of probability of serious bacterial infections in febrile children. A pilot study}

T Beattie, O Osman, D Brown, P Midgely

Edinburgh Sick Children's NHS Trust, Sciennes Road, Edinburgh EH9 $1 \mathrm{LF}$

Of 5021 patients seen over a period of nine weeks, $31 \%$ presented with features of an infectious illness of whom $41 \%$ were admitted to hospital. Altogether $43 \%$ of those admitted needed some form of treatment necessitating hospitalisation; $43 \%$ had blood culture taken and the rate of positive culture was $2.5 \%$.
Admitted children were significantly younger and had a higher temperature than those not admitted. More children were admitted when they were referred by their general practitioners than if they were self referred but the source of referra did not determine the need for hospital treatment.

The physician's scoring of the severity of illness tended to influence the decision to admit more than his/her scoring of the possibility of bacteraemia. Of many variables only the severity of illness scoring was predictive of the need for hospital treatment.

The scoring of severity was not predictive of the type of infection (bacterial $v$ nonbacterial) while the possibility scoring along with the absolute neutrophil count were the strongest predictors of the type of infection. Erythrocyte sedimentation rate, but not $C$ reactive protein, was significantly different between the two groups.

The absolute neutrophil count was most predictive of a positive blood culture, the tota white cell count and height of temperature were also significant predictors.

Conclusions - The absolute neutrophil count was the best predictor of a positive blood culture while the physician's subjective assessment of a febrile child solely was indicative of the need to admit the child for further assessment. A neural network will be used to help further analyse these criteria in the definitive study.

The improved delivery of analgesia to children attending accident and emergency

\section{Somers, D Hulbert}

Accident and Emergency Department, West Middlesex University Hospital NHS Trust, Twickenham Road, Isleworth, Middlesex TW7 6AF

The assessment of pain in children is not easy, especially at triage in the accident and emergency ( $A \& E$ ) department where nurses are constrained by time and usually unable to prescribe analgesia. Furthermore, A\&E doctors are often not confident or competent at dealing with pain in children.

Our impression was that children in pain attending our department could be better managed with the aid of simple measures.

Methods-A retrospective assessment of children attending the $A \& E$ department over the past two months was carried out, looking at diagnosis, time of triage, time seen by doctor, and the time that analgesia was prescribed. It was found that a large proportion of children in pain sat in the waiting roon after triage for long periods of time without being prescribed analgesia. To improve this the Wong-Baker faces pain rating scale was introduced at triage along with education for the nurses and doctors by the hospital pain control team and paediatric A\&E sisters. All children over 4 years of age in pain were assessed using this scale, and if it indicated moderate to severe pain a doctor was consulted to prescribe analgesia.

Results - We found that the introduction of the faces scale improved the assessment and delivery of analgesia to children over 4 years old in the $A \& E$ department and that $A \& E$ doctors became more confident about managing pain in these patients.

Conclusion-Simple measures at triage improve the care of children in pain. Allowing nurses to prescribe simple analgesia must now be considered. Highlighting pain management in children will hopefully improve the delivery of analgesia in adults also. There still remains the difficulty of assessing pain in children under 4 years.

Near patient testing for respiratory syncytial virus: a viable option for the accident and emergency department?

Audrey McKenzie, N Hallam, Elaine Murray, T Beattie

Accident and Emergency Department, Royal Hospital for Sick Children, Sciennes Road, Edinburgh EH9 $1 \mathrm{LF}$

Objective-To assess the performance of near patient testing for respiratory syncytial virus (NPT-RSV) in an accident and emergency (A\&E) setting.

Design-Prospective study of children under 2 years of age presenting with respiratory symptoms, comparing NPT-RSV in A\&E with conventional rapid testing (direct immunofluorescence, DIF) at the virology laboratory.

Main outcome measures-Sensitivity, specificity, positive and negative predictive values. Soundings were taken as to the acceptability of NPT-RSV to the staff performing it and to its perceived benefits in terms of patient management, infection control, and cost savings.

Results-NPT-RSV showed a sensitivity of $79 \%$, specificity of $97 \%$, positive predictive value of $98 \%$, and negative predictive value of $70 \%$, compared with DIF. The low sensitivity (compared with other studies) is partially explicable by operator error and study design (the first specimen of secretions from each child was sent for DIF, the second (taken immediately afterwards) was tested by NPTRSV). NPT-RSV was acceptable to staff and was judged to have clinical and financial benefits.

Conclusion-NPT-RSV is a viable option for $\mathrm{A} \& \mathrm{E}$ and has important, potential benefits. Positive results may be trusted, negative specimens require retesting at the virology laboratory.

Whiplash associated disorder arising from road traffic accidents to children

J M Hadfield, A Baxendale, S Cooper, S Wright, D W Yates

University Department of Emergency Medicine, Clinical Sciences Building, Hope Hospital, Salford M6 $8 \mathrm{HD}$

Introduction-Whiplash associated disorder (WAD) is a common occurrence in adults after a road traffic accident (RTA). Although classically used to describe a neck hyperextension injury after rear-end impact, neck pain may also follow lateral or frontal collisions. The aim of this study was to determine the incidence of WAD in children under 16 who were car occupants and involved in a RTA.

Patients and methods-Information was obtained from the CRASH (Co-ordinated Reduction of Accidents on Salford's Highways) project which contains linked hospital and police data on RTAs to children in Salford. The database contains information for the period May 1995 to December 1997. The anatomical site and severity of the injury were recorded in addition to age, sex, and type of accident. Telephone interviews with the child's parents were made after injury and subsequent disability determined. 
Table 4 Incidence (\%) of WAD at different ages in car occupants injured in RTAs

\begin{tabular}{|c|c|c|c|c|c|c|c|c|c|c|c|c|c|c|}
\hline Age (years) & 3 & 4 & 5 & 6 & 7 & 8 & 9 & 10 & 11 & 12 & 13 & 14 & 15 & Total \\
\hline Injured in RTA & 53 & 43 & 73 & 74 & 80 & 76 & 79 & 65 & 79 & 98 & 93 & 81 & 71 & 965 \\
\hline Car occupants & 29 & 15 & 24 & 25 & 20 & 16 & 21 & 19 & 21 & 26 & 15 & 19 & 23 & 273 \\
\hline WAD & 7 & 4 & 3 & 11 & 6 & 5 & 8 & 8 & 9 & 11 & 8 & 8 & 13 & 101 \\
\hline$\%$ WAD & 24.1 & 26.7 & 12.5 & 44.0 & 30.0 & 31.3 & 38.1 & 42.1 & 42.9 & 42.3 & 53.3 & 42.1 & 56.5 & 37.0 \\
\hline
\end{tabular}

Results-In the age group 3-15 years 965 children had sustained a RTA of which 273 $(28.3 \%)$ were identified as car occupants. In this second group $101(37.0 \%)$ presented with symptoms of WAD; $69.3 \%$ of this group were female. No child under the age of 2 years was diagnosed as having WAD. More than half the children $(56.4 \%)$ sustaining a WAD were in the 10-15 age group (see table 4). Fifty eight per cent of the accidents where a WAD was diagnosed had not been reported to the police. The main physical disabilities experienced two weeks after the incident were inability to dress without help, carry medium weight objects, and take part in sport. Parents also reported disturbed sleep patterns.

Conclusions-These data suggest that $37.0 \%$ of all RTAs involving children as car passengers result in the child sustaining a WAD. This is surprising in view of the fact that a literature search did not reveal any specific studies of the occurrence of WAD in children. The higher ratio of female to male patients has been previously reported in adults.

\section{Observer variation in measured ST seg- ment elevation}

Kurt D Kastendieck, Dan Tandberg, Scott Meskin

Department of Emergency Medicine, University of New Mexico School of Medicine, ACC 4-West, Albuquerque, New Mexico 87131, USA

Objectives-ST segment elevation (STSE) is used to make decisions about using thrombolytic therapy in patients with myocardial infarction (MI). This study was executed to assess interobserver and intraobserver variation in emergency physicians' measurements of STSE.

Design-A prospective, blinded, paired sample experiment.

Subjects-Emergency medicine students, residents, and faculty.

Interventions-Subjects were asked to measure STSEs in a packet of 40 electrocardiographic (ECG) complexes from patients with MI. Each packet consisted of 20 pairs of randomly ordered ECG complexes. Subjects were blinded to the duplicate nature of the pairs of complexes. First and second estimates of each ST segment measurement were subtracted; summary statistics were calculated for these differences. Agreement between paired measurements was analysed using weighted $\kappa$; differences among group medians were tested with the Kruskal-Wallis test.

Results -52 subjects completed the study and measured a total 2070 ST segments (1035 pairs). The mean absolute difference among all groups was $0.284 \mathrm{~mm}$ ( $95 \%$ confidence interval 0.267 to 0.302 ). The median was 0.2 $\mathrm{mm}$, the $80 \%$ ile was $0.5 \mathrm{~mm}$, and the $95 \%$ ile was $0.9 \mathrm{~mm}$. Students, residents, and faculty had similar median differences. Some subjects exhibited more intraobserver variation than others, and some complexes were associated with greater intraobserver variation than others $(p<0.0005)$. Statistical agreement between paired ST segment measurements was good $(\kappa=0.85 ; 95 \%$ confidence interval 0.826 to 0.873 ) Conclusion- $20 \%$ of the time intraobserver variation between first and second ST segment elevation measurements is more than $0.5 \mathrm{~mm}$. This could result in substantial misclassification of candidates for thrombolytic therapy.

Emergency department based HIV screening and counselling: experience with rapid and standard serological testing

Gabor D Kelen, Judy B Shahan, Thomas C Quinn, Project Educate Work Group

fohns Hopkins University School of Medicine, Department of Emergency Medicine, Baltimore, $M D$ 21287, USA

Context-Emergency departments are the only ready access to health care for many at risk for HIV.

Objective-To determine the feasibility of offering standard (EIA and western blot) or rapid HIV testing in an urban emergency room population.

Design-Voluntary prospective screening.

Setting-Large inner city hospital emergency department.

Patients-Consenting adults.

Protocol-Enrollment occurred during three distinct phases from 1993 to 1995. Rapid test results were divulged to patients. Patients enrolled in standard testing and those testing positive on the rapid test were asked to return for follow up confirmation and counselling.

Main outcomes-Proportion consenting; proportion HIV seropositive, cost of program. Results-Of 3048 patients approached, 1448 (48\%) consented, 981 to standard and 467 to rapid testing. Of these, $6.4 \%$ and $3.2 \%$, respectively, were newly identified as HIV seropositive. More than twice as many new infections were diagnosed among those discharged from the emergency department as those admitted (55 $v 21)$. Mean time to obtain results for the rapid assay performed in the hospital main laboratory was 107 (SD 52) minutes, with 55\% leaving the emergency department before receiving results. Rapid assays performed in the emergency department satellite laboratory required 48 (37) min with only $20 \%$ leaving before result availability. The follow up among HIV seropositives was $64 \%$ and $73 \%$ for the standard and rapid protocols, respectively $(p>0.20)$ The prearranged HIV clinic intake appointment was kept by $62 \%$. Cost per patient approached was $\$ 24$, and for per HIV+ identification, $\$ 794$ for the routine $v \$ 1495$ for the rapid.

Conclusion-Emergency department based HIV testing was well accepted, and detected a significant number of new HIV infections earlier than might have otherwise been. With relatively high HIV detection and return rates, it is evident that some emergency departments can play a major part in the national strategy of early HIV detection.

Serum lactate levels are associated with serum cytokine levels in emergency department patients

A P Tuttle, R M Nowak, M Maliraik, J Popovich, B Dereczyk, M Grzybowski, M C Tomlanovich

Henry Ford Hospital, Department of Emergency Medicine, 2799 West Grand Blvd, Detroit, Michigan 48202, USA

Introduction-Raised concentrations of serum lactate have been shown to have prognostic value in critically ill patients. Likewise, raised concentrations of various inflammatory cytokines have also been shown to be predictive of outcome. Serum concentrations of various cytokines were measured to determine if increases of these levels correlated with lactate concentrations.

Methods -991 adult patients presenting over a two week period to a tertiary care, inner city emergency department had blood drawn at triage; 478 patients had interleukin-6 (IL-6), intercellular adhesion molecule-1 (ICAM-1) tumor necrosis factor- $\alpha$ ( $\alpha$-TNF), interleukin $1-\beta$ (IL-1 $\beta$ ), and IL-1 receptor antagonist (IL1 ra) measured via ELISA. All patients had simultaneous serum lactate concentrations measured. Patients were stratified according to lactate levels; group I $<2.0 \mathrm{nmol} / 1$ (normal), group II 2.0-3.9 mmol/1 (equivocal), group III $\geqslant 4.0 \mathrm{mmol} / \mathrm{l}$ (abnormal).

Results-Mean (SD) concentrations are reported in table 5 . Bonferroni tests were used to test for differences between all groups for each cytokine.

Conclusions-These data suggests abnormal levels $(\geqslant 4.0 \mathrm{mmol} / \mathrm{l})$ of serum lactate are associated with significant increases of the serum cytokines IL-6, ICAM-1, $\alpha$-TNF, IL-1ra, but not IL-1 $\beta$. These data support an association between increases of some inflammatory mediators and serum lactate concentrations. These markers may have potential for early diagnosis and prognostic prediction. Further studies may show the comparative prognostic importance of these biomarkers. In addition, it will be necessary to evaluate whether cytokine levels.

Table 5

\begin{tabular}{lllllll}
\hline Group & \multirow{2}{*}{ No } & IL-6 $(\mathrm{pg} / \mathrm{ml})$ & ICAM-1 $(\mathrm{ng} / \mathrm{ml})$ & $a-T N F(\mathrm{pg} / \mathrm{ml})$ & $I L-1 \beta(\mathrm{pg} / \mathrm{ml})$ & $I L-1 \mathrm{ra}(\mathrm{pg} / \mathrm{ml})$ \\
\hline I & 290 & $27.1(154.8)$ & $235.8(157.9)$ & $2.05(6.87)$ & $3.72(39.4)$ & $1395(8432)$ \\
II & 150 & $46.1(208.0)$ & $282.3(219.1)$ & $2.93(6.51)$ & $0.13(0.47)$ & $1638(3964)$ \\
III & 38 & $303.7(1055)$ & $339.4(388.8)$ & $13.57(43.60)$ & $1.94(7.44)$ & $29695(122718)$ \\
p Value & & $<0.05$ I and III & $<0.05$ I and III & $<0.05$ I and III & NS & $<0.05$ I and III \\
& & $<0.05$ II and III & & $<0.05$ II and III & & $<0.05$ II and III
\end{tabular}


Free Paper Session IV: Audit, Training, Diagnosis, Delivery of Care, Tuesday September 29

Randomised trial with blinded comparison of minor injury care provided by accident and emergency nurse practitioners or senior house officers

M Sakr, J Angus, J Perrin, C Nixon, J Wardrope, J Nicoll

clo Accident And Emergency Department, Northern General Hospital, Sheffield S5 $7 A U$

Aim-To investigate the processes of care and outcomes of treatment in patients with minor injuries managed by nurse practitioners or by senior house officers.

Patients - 1500 patients over the age of 16 presenting with minor injuries that came within the scope of practice of nurse practitioners. Setting-A large urban teaching hospital accident and emergency (A\&E) department.

Design - A randomised study with double assessment of the patient (clinical and research) with comparison of process blinded. Patient satisfaction questionnaire and 28 day outcome questionnaire.

Main endpoints-Comparison between the clinical assessment and the research assessment of history taking, examination, use of radiography, interpretation of radiography, treatment, follow up, and advice. Comparison of patient reported outcome at 28 days and the need for unplanned follow up.

Findings-There were no significant differences between the nurse practitioners and the senior house officers in the accuracy of examination, adequacy of treatment and follow up, or requesting of $x$ rays. Radiography interpretation was similar in both groups.

There were no significant differences in the patient reported outcome at 28 days. However there was a statistically significant difference in the accuracy of history taking and in the need for unplanned follow up in favour of nurse practitioners.

Conclusions-Properly trained A\&E nurse practitioners, working within agreed guidelines, can provide care for patients with minor injuries that is equal or even superior to that provided by senior house officers.

Yesterday's sign: the normal human gag reflex

Chris Moulton, Vivian Tang

Accident and EmergencyDepartment, Royal Bolton Hospital, Minerva Road, Farnworth, Bolton BL4 OfR

Testing for a gag reflex is still advocated as a valid method of "assessing" the airway in an unresponsive patient. Absence of the gag reflex is often taken to be an indication for endotracheal intubation, although it is known that the reflex may be attenuated at all levels of the Glasgow coma scale. In 1982 Kulig, Rumack, and Rosen found that four out of 18 emergency room staff had no apparent response to pharyngeal stimulation, thus suggesting that a high proportion of normal individuals do not have a demonstrable gag reflex.

To test this hypothesis, we examined 160 healthy adults between 18 and 60 years old. The gag reflex could not be elicited in about one in eight of these subjects. An absent reflex is thus a very poor guide to the need for (and ease of) instrumentation of the airway.

The gag reflex has a large psychological component and is attenuated during swallowing. It may be that it is incorrectly classified as a protective reflex of the airway. The role of
Table 6

\begin{tabular}{ll}
\hline 1. None & $65(11)$ \\
2. Advice & $223(41)$ \\
3. To see general practitioner & $107(19)$ \\
4. General practitioner or nurse to visit & $127(23)$ \\
5. At risk & $26(5)$ \\
6. To reattend A\&E & $3(1)$ \\
Total & 551
\end{tabular}

Values are number (\%).

the gag reflex in emergency medicine will be discussed as will other methods of assessing the degree of airway protection.

Next day telephone follow up of the elderly-a quality assurance and critical incident monitoring tool in accident and emergency medicine

H D M Poncia, J Ryan, M Carver

Department of Accident and Emergency Medicine, Royal Sussex County Hospital, Eastern Road, Brighton BN2 5BE

Introduction-Patients over the age of 75 years comprise an increasing proportion of accident and emergency (A\&E) department attendances. The case mix associated with this population group frequently have complex medical and psychosocial problems which need to be addressed before discharge. For instance, elderly patients who present with minor complaints may have communication and mobility problems overlooked.

Aims - The aim of this study was to identify the needs of an elderly population the day after discharge from an A\&E department. The objective was to produce recommendations for A\&E staff which would help them identify, before discharge from the A\&E department, patients who may be in an "at risk" category. Methods-A structured questionnaire was designed to collect information on elderly patients who attended the A\&E department the previous day. Any patient over the age of 75 years who was seen in the $A \& E$ department and discharged the previous day or any patient admitted to the departmental short stay ward overnight were included in the study. A total of 551 patients or their carers were contacted by telephone. Information was recorded on a database and analysed using Epi-Info version 5.0. Interventions initiated by the community nurse were scored from 1 to 6 based on the level of input required.

Results-Table 6 shows the types of interventions required the day after attendance at $A \& E$ as judged after a telephone interview by a community liaison nurse.

Conclusions-Telephone follow up of the over 75 s attending our $A \& E$ department identified a number of areas where care could be improved before discharge. Furthermore this low cost, high quality intervention has the potential for decreasing inappropriate return visits to the department by a group of patients with relatively high dependency. A teaching package based on the information obtained has been prepared for $A \& E$ staff.

Five year survival of patients discharged from the emergency department with acute chest pain

C G McMahon, S Hollis, D W Yates, F A Campbell

University Department of Emergency Medicine, Hope Hospital, Salford M6 8HD

Objective-To evaluate the short term survival of patients discharged from the emergency department with acute chest pain.
Design-Longitudinal follow up study of survival as an outcome measure.

Methods-The study cohort included all patients over the age of 30 years who attended the emergency department between January 1993 and June 1994 with acute chest pain who had electrocardiography performed but were subsequently discharged directly from the emergency department. Demographic details of this group were registered with the Office for National Statistics to identify the time and cause of death of any members of the group. The survival time was censored at 31 December 1997 for those who had not been registered dead at this time.

The age at entry and the time at risk (until death or to the end of the study period) were calculated for each subject. These were used to calculate Kaplan-Meier survival curves with confidence intervals. The expected survival experience of the entire cohort was calculated from their age at entry and the local population mortality rates. The confidence limits on the Kaplan-Meier survival curve were used to determine whether actual survival was significantly different from expected.

Results-786 patients were entered into the study. The age and sex breakdown was as follows: males $<65$ years, $n=374$; females $<65$ years, $n=205$; males $>65$ years, $n=92$; females $>65$ years, $n=115$. There were 139 registered deaths, $41 \%$ of which were cardiac in origin compared with an expected $28.4 \%$. The risk of cardiac deaths in males was particularly high accounting for $51.5 \%$ of deaths in those less than 65 years, and $48.6 \%$ of deaths in those over 65 years compared with an expected $30.7 \%$ and $30.9 \%$ respectively. Female cardiac death rates were not significantly increased. The overall observed five year survival rate was $81.5 \%$ (95\% confidence interval $78.6 \%$ to $84.5 \%$ ), significantly lower then the expected rate of $88.9 \%$ ( $\mathrm{p} 0.01$ ).

Conclusion-There is good evidence that patients with coronary syndromes benefit from aspirin, $\beta$ blockers, and lipid lowering treatment. This study suggests that the initial assessment of patients with chest pain is not optimal. There is a need for better risk stratification of this group. Evaluation of patients presenting to the emergency department with chest pain should be a first step in a more integrated approach to the management of coronary disease.

Does an alternative source for fever 'rule-out' the diagnosis of infective endocarditis in the emergency department?

Peter M Hill, Roderick I Bahner, Gabor D Kelen, Richard E Rothman

Fohns Hopkins University, School of Medicine, Department of Emergency Medicine, Baltimore, $M D, U S A$

Objective-Infective endocarditis (IE) is a diagnosis which must be considered in every febrile patient with intravenous drug use (IDU) who presents to the emergency department. Appropriate management of this population mandates evaluation of the utility of the Duke criteria. This study determines whether the Duke criterion for "rejected IE" can be applied in the emergency department when an alternative source of fever is identified.

Methods-A 12 month retrospective chart review of all patients presenting with a history of IDU and fever $\left(\geqslant 38^{\circ} \mathrm{C}\right)$ to a large, inner city academic emergency department was conducted. Charts were collected as part of the Drug Abuse Warning Network Project. All patients with a history of IDU, fever, and 


\begin{tabular}{llll}
\hline & 1996 & 1997 & 1998 \\
\hline \# EM programs with vacancies (\%) & $6 / 128(5)$ & $10 / 130(8)$ & $24 / 126(19)$ \\
\# Unmatched EM positions (\%) & $13 / 1030(1)$ & $24 / 1065(2 \%)$ & $66 / 1121(6)$ \\
\# Applicants ranking only EM programs/at least 1 EM program: & & $728 / 955$ & $694 / 949$ \\
US graduates & $788 / 1091$ & $360 / 543$ & $291 / 462$ \\
Independent applicants & $346 / 515$ & $1088 / 1498$ & $985 / 1411$ \\
$\quad$ Total applicants & $1134 / 1606$ & & \\
Unmatched US seniors (\%) & $102 / 788(13)$ & $53 / 728(7)$ & \\
Breakdown of filled positions: & 1030 & 1065 & 1098 \\
Total EM position & $854(83)$ & $829(78)$ & $824(75)$ \\
Filled by US graduates (\%) & $163(16)$ & $212(20)$ & $208(19)$ \\
Filled by independent applicants (\%) & $1041(98)$ & $1032(94)$ \\
Total filled (\%) & $1017(99)$ & & \\
\hline
\end{tabular}

$\mathrm{EM}=$ emergency medicine.

hospital admission were included. Data extracted from the emergency department chart and discharge summary included presence of alternative source of fever upon presentation bacteraemia, echocardiographic findings, and discharge diagnosis.

Results-225 patients met inclusion criteria 34 were found to be bacteraemic and 191 were not bacteraemic. All non-bacteraemic patients met Duke rejection criteria upon discharge from the hospital. Altogether 18/34 (53\%) bacteraemic patients had echocardiograms performed and met criteria for definite IE; $5 / 18(28 \%)$ of these patients presented with an alternative source of fever. Sixteen of the 34 bacteraemic patients did not undergo echocardiography and are classified as having possible endocarditis; 13 of these patients had an alternative source of fever identified and three had no other source identified.

Conclusion-A large percentage $(28 \%)$ of patients classified as having definite IE by strict Duke criteria had another explanation for their fever upon presentation to the emergency department. The Duke criteria may be internally inconsistent in that they suggest rejecting the diagnosis of IE in certain instances where IE is later definitively demonstrated.

The 1998 US National Residency Matching Program results in emergency medicine

Louis S Binder

University of Illinois at Chicago College of Medicine, USA

Objectives-To present the results of the 1998 National Residency Matching Program (NRMP) residency match in emergency medicine, compared with data from 1996 and 1997.

Design-Prospective observational study.

Subjects-All applicants to emergency medicine residency programs through the NRMP $100 \%$ sample which includes all applicants and programs that entered the 1996-98 matches.

Results-Table 7 shows the results.

Conclusions-(1) Emergency medicine has exhibited continued growth in its US academic base, with an increase of 56 entry level positions from 1997 to 1998 (5.3\% increase) (2) The US applicant pool contracted by $10 \%$ between 1996 and 1997, and by an additiona $5 \%$ from 1997 to 1998 . The independent applicant pool contracted by about $15-20 \%$ in 1998 in the face of expansion of the training base. (3) The percentage of applicants attempting to enter the field in excess of the training base ranged from $-12 \%$ to $+26 \%$ (depending on parameters used to define the applicant pool). The "unmatched" rate for US seniors attempting to enter the field $(4.7 \%)$ is lower in 1998 than that for the overall NRMP match $(5.3 \%)$.
Now that we have the Utstein template, are we using it?

David C Cone ${ }^{\star}$, David S Jaslow ${ }^{\star \star}$, Thomas A

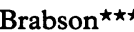

*Department of Emergency Medicine, Allegheny University of the Health Sciences, Philadelphia, **Division of Emergency Medicine, Temple University School of Medicine, Philadelphia, and ***Department of Emergency Medicine, Alber Einstein Medical Center, Philadelphia, PA, USA

Objectives-To examine the extent to which the Utstein template has been used for out-ofhospital cardiac arrest (OOHCA) research since its release in 1991. The template was developed in an effort to standardise OOHCA research and reporting.

Methods-All issues of six emergency medicine/emergency medical service journals (Academic Emergency Medicine, American fournal of Emergency Medicine, Annals Emergency Medicine, fournal of Emergency Medicine, Prehospital Disaster Medicine (1992-96), and Prehospital Emergency Care (1997)) from 1992 to the present were examined, as were all peer reviewed OOHCA papers from other journals found on MEDLINE and CINAHL. All OOHCA research articles were examined to determine whether use of the Utstein template was indicated and if so, whether it had been used. Difference of proportions ( $95 \%$ confidence intervals) was used to compare rates from US and non-US institutions.

Results-All 143 OOHCA research articles identified were examined. Of these, $41(29 \%)$ studied topics such as OOHCA ethics that were not amenable to the template. These were excluded, leaving 102 for which the Utstein template was indicated. Forty one $(40 \%)$ used the Utstein template, and 61 $(60 \%)$ did not. There was no difference in rates between papers from sites in the US (18/ $48,38 \%)$ and elsewhere $(23 / 54,43 \%)$; see table 8. (Similar table results were obtained when date of submission was used instead of date of publication. However, no date of submission was listed for 25 papers.)

Conclusions-Six years after the release of the Utstein template for OOHCA research, fewe than $60 \%$ of OOHCA research articles actually use the template.

Table 8 Number (\%) of OOHCA research papers using Utstein template

\begin{tabular}{llll}
\hline Year & All papers & US & Non-US \\
\hline 1992 & $0 / 6(0)$ & $0 / 4(0)$ & $0 / 2(0)$ \\
1993 & $4 / 25(16)$ & $2 / 16(13)$ & $2 / 9(22)$ \\
1994 & $7 / 13(54)$ & $5 / 8(63)$ & $2 / 5(40)$ \\
1995 & $14 / 27(52)$ & $6 / 10(60)$ & $8 / 17(47)$ \\
1996 & $8 / 17(47)$ & $3 / 6(50)$ & $5 / 11(45)$ \\
1997 & $8 / 14(57)$ & $2 / 4(50)$ & $6 / 10(60)$ \\
Total & $41 / 102(40)$ & $18 / 48(38)$ & $23 / 54(43)$ \\
\hline
\end{tabular}

Free Paper Session V: Resuscitation, Wednesday 30 September

Missed resuscitation opportunities following drowning

J P Wyatt, G Tomlinson, A Busuttil

Accident and Emergency Department, Royal Infirmary of Edinburgh, Lauriston Place, Edinburgh EH3 9YW

Introduction-Immersion under water (particularly cold water) is compatible with long term survival, even when the period of immersion is relatively long. Guidelines for resuscitation after immersion stress the importance of prolonged resuscitation using advanced life support techniques. This study examines the extent to which individuals who died after drowning in south east Scotland were resuscitated.

Methods-The circumstances of deaths due to drowning in Lothian and Borders regions during seven years (1991-97) were reviewed, with particular reference to whether resuscitation was performed. Data sources included the procurator fiscal records, necropsy reports prepared by the Forensic Medicine Unit, police, ambulance, and hospital records.

Results-95 deaths (69 males, 26 females) from drowning occurred in the following sites: sea, 35; bath, 15; flowing freshwater (rivers, streams), 26; still freshwater (reservoirs, lochs, canals, ponds), 19 . Twenty two $(23 \%)$ of the drowning incidents were witnessed, $73(77 \%)$ were unwitnessed.

Twenty eight individuals were recovered from water within an hour of them having been last seen alive, 13 having had witnessed accidents, 15 having had unwitnessed accidents. Six individuals were not resuscitated at the scene by the police or other emergency services, despite being seen alive within the previous hour. A further five individuals were initially resuscitated, but declared dead by a general practitioner at the scene within one hour of being known to be alive.

Conclusions-The results demonstrate that members of the emergency services, including general practitioners, are failing to both initiate pre-hospital resuscitation and to continue this to hospital for victims of near drowning. These results suggest that there may be some potential to reduce the death rate from drownings in south east Scotland by improving resuscitation. Police officers, general practitioners, other members of the emergency services, and the public should be educated about the need to commence basic life support on those retrieved from water.

Direct assessment of tissue oxygen delivery, availability, and utilisation in patients with shock

Charles B Cairns, David Jones, Gregory Bell, David Nyman, Paul Bender

Colorado Emergency Medicine Research Center, University of Colorado School of Medicine, University of Colorado Health Sciences Center, Denver, Colorado, USA

Introduction-Shock is characterised by reduced tissue oxygen delivery. Near infrared spectroscopy (NIRS) allows for continual monitoring of tissue oxygen delivery (oxyhemoglobin; $\mathrm{Hbo}_{2}$ ), availability (oxygen saturation; $\mathrm{StO}_{2}$ ), and utilisation (mitochondrial cytochrome a, $a_{3}$ redox state). Normally, $\mathrm{Hbo}_{2}$ and $\mathrm{a}, \mathrm{a}_{3}$ redox states are tightly coupled decoupling is a sign of mitochondrial dysfunction. 


\begin{tabular}{|c|c|c|c|c|c|}
\hline \multirow[b]{2}{*}{ Group } & \multirow[b]{2}{*}{ ROSC rate } & \multirow{2}{*}{$\begin{array}{l}\text { CPR onset to ROSC } \\
\text { time (min) }\end{array}$} & \multicolumn{3}{|c|}{$C P P(m m H g)$} \\
\hline & & & $11 \mathrm{~min}$ & $12.5 \mathrm{~min}$ & $15.5 \mathrm{~min}$ \\
\hline Control & $0 / 7$ & - & $2.0(3.8)$ & $3.7(4.1)$ & $3.0(3.3)$ \\
\hline SAAP-C1 & $6 / 6^{\star}$ & $9.7(2.3)$ & $4.0(3.3)$ & $32.3(5.3) \dagger$ & $38.7(5.7) \dagger$ \\
\hline SAAP-C2 & $7 / 7^{\star}$ & $9.8(1.0)$ & $1.7(1.4)$ & $32.7(4.8) \dagger$ & $33.7(5.1) \dagger$ \\
\hline SAAP-PD & $7 / 7^{\star}$ & $7.7(1.2)$ & $1.4(1.1)$ & $34.7(8.3) \dagger$ & $39.0(13.3) \dagger$ \\
\hline
\end{tabular}

intubations-final report of the National Emergency Airway Registry pilot project Ron M Walls*, Robert J Vissers ${ }^{\star \star}$, Mark J Sagarin ${ }^{\star}$, John C Sakles ${ }^{\star \star}$, Steven Bernstein $^{\star \star \star \star}$, Erik D Barton ${ }^{\star}$, Richard E Wolfe ${ }^{\star}$ on behalf of the NEAR investigators

${ }^{\star}$ Brigham and Women's Hospital, Department of Emergency Medicine, Harvard Medical School, **UNC Chapel Hill, Department of Emergency

${ }^{\star} \mathrm{p}<0.001 v$ control, Fisher's exact test.

tp $<0.05 v$ control, analysis of variance.

Hypothesis-Physiological changes during clinical shock states lower tissue oxygen delivery $\left(\mathrm{Hbo}_{2}\right)$, availability $\left(\mathrm{Sto}_{2}\right)$, and utilisation (a, $a_{3}$ redox state).

Study design-Prospective, observational study of shock patients (initial systolic blood pressures (SBP) $<90 \mathrm{~mm} \mathrm{Hg}$ ) undergoing resuscitatation in either the emergency department or intensive care unit.

Measurements-All patients were continuously monitored for NIRS derived $\mathrm{Hbo}_{2}, \mathrm{Sto}_{2}$, and the cytochrome $a, a_{3}$ redox state as well as standard clinical recordings such as heart rate, SBP, and arterial oxygen saturation.

Results - 12 patients (four women) ranging in age from 26 to 79 years (mean 57) were enrolled. Changes in cerebral and deltoid $\mathrm{HbO}_{2}$ and $\mathrm{StO}_{2}$ preceded changes in SBP after administration of fluids, vasopressors, antiarrhythmics, and Trendelenburg positioning. Changes in systolic blood pressure correlated with changes in cerebral $\mathrm{StO}_{2}, \mathrm{Hbo}_{2}$ $(r=0.88 ; \mathrm{p}<0.05)$, and the cytochrome $\mathrm{a}, \mathrm{a}_{3}$ redox state $(r=0.73 ; \mathrm{p}<0.05)$. In addition, two patients (hepatorenal dysfunction, disulfiram reaction) had evidence of decoupling, consistent with impaired mitochondrial electron transport.

Conclusions-(1) Shock states and their management affect tissue oxygen delivery, availability, and utilisation. (2) Shock can also be complicated by concurrent dysfunction of mitochondrial oxidative metabolism. (3) NIRS is a sensitive detector of both clinical status and tissue oxygenation, which may prove useful in the management of emergency department shock patients.

Comparison of three perfusion methods for performing selective aortic arch perfusion during cardiac arrest

James E Manning, D Neil Batson, Chris Pugh, Charles Cheek, Robert A Mueller

Department of Emergency Medicine, School of Medicine, University of North Carolina at Chapel Hill, USA

Objective-Selective aortic arch perfusion (SAAP) is an artificial perfusion technique for treating cardiac arrest. Three different SAAP methods were compared to evaluate their relative effects on coronary perfusion pressure (CPP) and return of spontaneous circulation (ROSC).

Methods-Twenty eight dogs with micromanometer and SAAP catheters underwent 10 min of ventricular fibrillation before mechanical cardiopulmonary resuscitation (CPR). Epinephrine (AoE) $0.01 \mathrm{mg} / \mathrm{kg}$ was given via the aortic arch at $12 \mathrm{~min}$ and then at $3 \mathrm{~min}$ intervals. Defibrillation attempts were at 13 min then $\mathrm{q} 1 \mathrm{~min}$ as needed up to the $30 \mathrm{~min}$ mark. Controls $(n=7)$ received $C P R$ and $A o E$ alone. SAAP groups received CPR, AoE, and SAAP with $450 \mathrm{ml}$ over 45 seconds of saline and oxygenated autologous blood (oxyAB) at $12 \mathrm{~min}$ and then oxyAB at $15.5 \mathrm{~min}$ and $\mathrm{q} 1$ min thereafter until ROSC.
SAAP-C1 $(n=7)$ received continuous nonpulsatile SAAP with continued CPR. SAAP-C2 $(n=7)$ received continuous nonpulsatile SAAP without CPR. SAAP-PD $(n=7)$ received pulsed diastolic SAAP during continued CPR with perfusate pulses infused only during the diastolic phase of CPR.

Results-Table 9 shows ROSC rate, time from onset of CPR to ROSC, and CPP data. ROSC data in one SAAP-C1 was lost due to apparatus malfunction.

Conclusion-All three SAAP methods were effective. SAAP-PD tended to result in more rapid ROSC, but this was not explained by higher CPP.

Rapid defibrillation by non-traditional responders: the casino project

Terence D Valenzuela ${ }^{\star}$, Henry S Bjerke ${ }^{\star \star}$ Lani L Clark ${ }^{\star}$, Richard Hardman ${ }^{\star \star \star}$, Daniel W Spaite ${ }^{\star}$, Graham Nichol ${ }^{\star \star \star \star}$

${ }^{\star}$ University of Arizona, Tucson, Arizona, ${ }^{\star \star}$ University of Nevada, Las Vegas, Nevada, ${ }^{\star \star \star}$ Clark County Fire Department, Las Vegas, Nevada, USA, and ${ }^{\star * \star *}$ Loeb Research Institute, Ottawa, ON, Canada

Objective-To determine whether victims of out-of-hospital ventricular fibrillation can be successfully resuscitated by non-traditional emergency responders using automatic external defibrillators (AEDs).

Design-Prospective experimental, consecutive case series.

Setting-Eighteen gaming establishments.

Subjects-Individuals who collapsed on premises of participating establishments from 1 May 1996 to 31 December 1997.

Intervention-Security officers were instructed in manual cardiopulmonary resuscitation (CPR) and the use of AEDs. AEDs were placed to meet a target collapse-todefibrillation interval of $\leqslant 3 \mathrm{~min}$

Results-AEDs were used on 22 individuals seven were determined not to be cardiac arrests. Of the 15 cardiac arrests, 11 were witnessed, the victims' mean age was 57 years, and ventricular fibrillation was the initial cardiac rhythm in 10 patients. Seven of the 15 $(46 \%)$ arrest victims survived to hospital discharge. Seven of the 10 cases $(70 \%)$ of ventricular fibrillation survived to hospital discharge. Time interval data was available on seven of the 10 cases of ventricular fibrillation: collapse-to-attachment of AED: $1.4 \pm 1.3$ min; collapse-to-first shock: $2.2 \pm 1.1 \mathrm{~min}$; collapse-to-arrival of traditional emergency medical service responders: $6.6 \pm 2.6 \mathrm{~min}$ Conclusion-Non-traditional responders trained in CPR and the operation of automatic external defibrillators, achieved shorter collapse to defibrillation intervals and higher survival to discharge than ever previously reported for out-of-hospital cardiac arrest. Rapid defibrillation by non-traditional responders is a viable strategy for significantly improving survival after out-of-hospital cardiac arrest due to ventricular fibrillation.
Medicine ${ }^{* \star} U C$ Davis Medical Center, Department of Emergency Medicine, ${ }^{* * * S t ~ L u k e s-~}$ Roosevelt Medical Center, USA

Objective-To characterise emergency department airway management in the US, including success and complication rates.

Methods-Prospective observational study of 1288 emergency department intubations in 11 US teaching hospitals recorded in the database during the pilot phase (8/96 to 8/97) for the National Emergency Airway Registry (NEAR).

Results-1288 intubations were registered over the pilot period; 984 were adults, 144 children $<18$ years, 160 age unknown. Rapid sequence intubation (RSI), oral intubation with sedation only (SED), oral intubation without medications (NOM), and nasal intubation (NTI) were the first method in $79 \%, 8 \%, 11 \%, 2 \%$ of adults and $80 \%, 6 \%$, $14 \%, 0 \%$ of children. Success rates in all patients in $1, \leqslant 2, \leqslant 3$ attempts were $77 \%$, $90 \%, 95 \%$ for RSI, $57 \%(\mathrm{p}<0.0001$ when compared with RSI), $79 \%$ ( $p<0.01), 87 \%$ $(\mathrm{p}<0.001)$ for SED, 66\% $(\mathrm{p}<0.01), 81 \%$ $(\mathrm{p}<0.01), 88 \%(\mathrm{p}<0.001)$ for NOM, and $60 \% \quad(p=N S), \quad 65 \% \quad(p<0.001), \quad 65 \%$ $(\mathrm{p}<0.00001)$ for NTI. By discipline, intubations of adults and children were performed by emergency medicine $(93 \%, 57 \%)$, paediatric emergency medicine $(0 \%, 22 \%)$, paediatrics $(0 \%, 9 \%)$, anaesthesia $(2 \%, 5 \%)$, surgery $(1 \%, 2 \%)$, IM $(2 \%, 0 \%)$, and other $(2 \%, 6 \%)$. Complication rate overall was $13 \%$, and by method $12 \%$ RSI, $20 \%$ SED, $12 \%$ NOM, and $26 \%$ NTI. Serious complications were rare $(3 \%)$.

Conclusions-Most emergency department intubations in these 11 centres were performed by emergency physicians, and most were done using RSI. Success rates vary by method, and are generally superior with RSI. Serious complications are rare.

Free Paper Session VI: Trauma II, Wednesday 30 September

Derivation of prediction rules for posttraumatic lung injury

T H Rainer ${ }^{\star}$, R A Cocks ${ }^{\star}$, C Y Man ${ }^{\star}$, N K Cheung $^{\star}$, P Lam ${ }^{\star \star}$, E Wong ${ }^{\star \star}$

${ }^{\star}$ Accident and Emergency Medicine Academic Unit, and ${ }^{*}$ Centre for Clinical Trials and Epidemiological Research, Chinese University of Hong Kong and Prince of Wales Hospital, Shatin, Hong Kong

Background - Lung injury is well recognised after trauma and in severe cases is associated with high mortality. Early prophylactic interventions may prevent post-traumatic pulmonary complications but identifying at risk patients remains elusive. The aim of this study was to derive a simple, highly sensitive prediction rule to correctly classify those subjects at risk of post-traumatic acute pulmonary complications.

Methods -96 adults triaged to the resuscitation room of an emergency department in a university hospital after blunt trauma were 
studied prospectively. Each patient was assessed for 25 clinical and haematological parameters by one emergency research physician. Data were analysed by Fisher's exact test and Mann-Whitney $U$ test. Eleven potential variables were reanalysed using a classification and regression tree. The prediction value positive (PV+ (95\% confidence intervals, CI)) was determined to show the correctness of the classification rate. The outcome measures were acute lung injury (ALI) and acute respiratory distress syndrome (ARDS).

Results - The prediction rules included injury severity score (ISS) $>27$, packed cell volume $<0.37$, total leucocyte count $>15.35$, and revised trauma score (RTS) $<7.227$.

In model $1, \mathrm{ALI}(\mathrm{n}=7)$ was correctly predicted (PV+ 70\%; 95\% CI $34.8 \%$ to $93.3 \%$ ) by ISS $>27.5$ and packed cell volume $<0.37$. ALI could be excluded (PV+ $100 \% ; 95 \%$ CI $96.4 \%$ to $100 \%)$ by either ISS $<27(n=74)$, or ISS $>27$ and packed cell volume $>0.37(n=8)$.

Model 1 correctly classified ALI in 89/92 (96.7\%; $95 \%$ CI $90.8 \%$ to $99.3 \%$ ) cases. The sensitivity was $100 \%(95 \%$ CI $65.2 \%$ to $100 \%$ ) and specificity $96.5 \%$ (95\% CI $90 \%$ to $99.3 \%)$.

In model 2, ARDS ( $n=4)$ was correctly predicted (PV+ $80 \%$; 95\% CI $28.4 \%$ to $99.5 \%$ ) by a combined ISS $>27.5$, packed cell volume $<0.37$ and $\mathrm{RTS}>7.227$. ARDS was excluded by either an ISS $<27.5(n=74$; PV+ $100 \% ; 95 \%$ CI $96 \%$ to $100 \%$ ) or an ISS $>27.5$ and packed cell volume $>0.37(\mathrm{n}=8$; PV+ $100 \% ; 95 \%$ CI $68.8 \%$ to $100 \%$ ) or packed cell volume $<0.37$, RTS $<7.2$ and ISS $>27$ ( $n=5$; PV+ $100 \% ; 95 \%$ CI $54.9 \%$ to $100 \%$ ) combination. Model 2 correctly classified ARDS in 90/92 (97.8\%; 95\% CI $92.4 \%$ to $99.7 \%$ ) cases with a sensitivity of $80 \%(95 \%$ CI $28.4 \%$ to $99.5 \%)$ and a specificity of $98.9 \%(95 \%$ CI $93.8 \%$ to $100 \%)$.

Conclusions-Practical highly sensitive prediction rules for post-traumatic pulmonary injury have been derived. These rules require prospective validation.

Better non-invasive assessment of the circulatory status in recently injured adults

Fiona E Lecky

Accident and Emergency Department, Hope Hospital, Stott Lane, Salford M6 8 HD

Objective-To evaluate whether some combination of non-invasive tests performs better than pulse rate and blood pressure in classifying the circulatory status of recently injured adults.

Design-Prospective study of non-invasive, point of care, diagnostic test performance.

Setting-UK emergency department and South African trauma unit.

Subjects-239 recently injured adults all requiring inpatient hospital care.

Tests studied-Pulse rate, systolic mean and diastolic arterial pressure, shock index, Glasgow coma score (GCS), respiratory rate, stroke distance, patient appearance, blood glucose, haemoglobin, oximeter signal strength, rate pressure product, and temperature. This combination of tests was measured within an 3-8 minute time frame on each patient.

Main outcome measures-Each test's ability to predict the circulatory status according to whether the simultaneously measured plasma lactate is $\geqslant$ or $<2.5 \mathrm{mmol} / 1$. Compari- sons of sensitivity at fixed specificity were made by McNemar's test for paired data.

Results-No single test could achieve a rule in specificity of $75 \%$ without a high (>30\%) false negative rate of shock detection. Recursive partitioning showed that a combination of GCS, haemoglobin, and shock index achieved a significantly lower false negative rate. This combination was significantly $(p<0.05)$ more sensitive than an equally specific pulse rate and blood pressure combination.

Conclusion-A combination of GCS, shock index, and haemoglobin appears to better predict the circulatory status of recently injured adults than any combination of pulse rate and blood pressure.

\section{A stab in the dark: evaluating TRISS as a predictor of UK penetrating trauma out-} comes

N Nichol, R Crawford, K Little, A Ireland, D Beard, J Henry

Scottish Trauma Audit Group, Royal Infirmary of Edinburgh, Lauriston Place, Edinburgh EH3 $9 Y W$

The Scottish Trauma Audit Group was set up in 1991 to audit the management of injured patients in Scotland. The W statistic measures a hospital's performance in terms of unexpected survivors or deaths as predicted using TRISS methodology. In 1995, it was observed that there was a statistically significant difference in the penetrating trauma W statistic for two comparable Scottish city centre hospitals.

A study was undertaken to examine the management of 404 penetrating trauma patients who presented during 1 February 1992 to 31 October 1996. Case notes were reviewed for the extraction of additional data (for example pre-existing medical conditions). All unexpected outcomes and deaths were reviewed by an independent panel of consultant surgeons who were blinded to patient details. They assessed clinical management to correlate the TRISS probability of survival with the actual injuries and clinical condition of the patients.

There were 272 patients admitted to hospital A and 132 to hospital B with 67193 and 91769 annual new accident and emergency attendances respectively (1997). Both hospitals had similar proportions of patients with injury severity scores between 16 and 75 (hospital A: 60, 22\% $v$ hospital B: 25 , $19 \%)$. A total of 97 patients required a laparotomy (hospital A: 63, 23\% v hospital B: $34,26 \%$ (NS)). Significantly more of the hospital A patients had penetrating chest trauma $(142,52 \%$ v $41,31 \% ; \mathrm{p}<0.05)$. There was a significant difference in crude mortality (hospital A: 37, 14\% $v$ hospital B: 7, 5\%; p<0.05) There was a significant difference between the two hospitals in the number of unexpected outcomes identified using TRISS methodology. In hospital A there was on unexpected survivor and nine unexpected deaths, and in hospital $B$ there were two unexpected survivors and no unexpected deaths.

The methodology of trauma scoring and its use as a predictor of UK penetrating trauma outcomes are reviewed. Management problems were identified and recommendations made regarding the optimal management of patients with penetrating trauma.
Predicting postconcussive syndrome after minor head injury in the emergency department

Jeffrey J Bazarian*, Tony Wong ${ }^{\star \star}$, Michael Harris*, Neil Leahey*, Sohug Mookerjee*, Mary M Dombory $\star \star$

${ }^{\star}$ Department of Emergency Medicine, University of Rochester Medical Center, ${ }^{\star *}$ Brain Injury Rehabilitation Unit, St Mary's Hospital, Rochester, NY, USA

Objective-To determine if clinical variables or neurobehavioural test (NBT) scores obtained in the emergency department within 24 hours of minor head injury (MHI) predict the development of postconcussive syndrome at one, three, and six months.

Methods-Prospective, observational study of a convenience sample of 71 patients with $\mathrm{MHI}$ and 60 orthopaedic controls presenting to the emergency department of a university teaching hospital between February 1996 and October 1997. MHI was defined as loss of consciousness $<10 \mathrm{~min}$ or amnesia, Glasgow coma score 15 , no skull fracture or new neurological focality on $\mathrm{PE}$, and no brain injury on computed tmography (if done). Clinical and demographic data were collected with a standardised patient encounter form. All patients received a $20 \mathrm{~min}$ NBT battery in the emergency department: neurobehavioural cognitive status exam, trailmaking $A$ and $B$ digit span test (forward and back), symbol digit modality test, and Hopkins verbal learning $\mathrm{A}$ and $\mathrm{B}$. Telephone follow up was done at one, three, and six months to determine if patients met the DSM IV definition of postconcussive syndrome.

Analysis-Stepwise, multivariate, logistic regression.

Results-Incidence of postconcussive syndrome among $\mathrm{MHI}$ patients was $58 \%$ at one month, $43 \%$ at three months, and $25 \%$ at six months. Predictors of postconcussive syndrome at one month were female gender (odds ratio $(O R)=7.8 ; 95 \%$ confidence interval $(C I)=41.6$ to 1.82$)$ ), presence of both retrograde and anterograde amnesia $(\mathrm{OR}=0.055 ; \mathrm{CI}=0.002$ to 0.47$)$, digit span forward scores $(\mathrm{OR}=0.748 ; \mathrm{CI}=0.52$ to 1.03 ), and Hopkins verbal learning $A$ scores $(\mathrm{OR}=0.786 ; \mathrm{CI}=0.65$ to 0.91$)$; at three months, presence of both retrograde and anterograde amnesia $(\mathrm{OR}=0.13 ; \mathrm{CI}=0.0$ to 0.93 ), digit span forward scores ( $O R=0.744$ $\mathrm{CI}=0.58$ to 0.94 ). No variables fit the model at six months. Ninety two per cent of males scoring $>25$ on Hopkins verbal learning A did not have postconcussive syndrome at one month, and $89 \%$ of females scoring $<9$ on digit span forward did have postconcussive syndrome at one month.

Conclusions-Gender and two simple NBTs can help predict postconcussive syndrome a one and three, but not six months after MHI.

Neurological consequences of multiple episodes of fluid percussion head injury sustained while intoxicated on chronic and acute alcohol exposed rodents Michelle Biros, Denise Kukielka

Hennepin County Medical Center, Minneapolis, $M N, U S A$

Multiple episodes of minor head trauma are frequent among intoxicated chronic alcoholic patients.

Objective-To determine the neurological consequences of multiple episodes of mild fluid percussion head injury (FPI) on intoxicated chronic (CR) and non-chronic (NCR) alcoholic rodents. 
Methods-This was a whole animal survival prospective study. Two hours before experimentation, CR and NCR alcoholic rodents received intoxicating levels of $95 \%$ ethyl alcohol $(3 \mathrm{~g} / \mathrm{kg})$ or normal saline by NG. Animals were further randomised to mild FPI $(1.7 \pm 0.4$ ATA) or no injury (minimum $n=9$ based on a priori power calculations). All animals underwent identical operative preparation. Injured animals received three FPIs (once every four days). Reflex recovery time (RRT) was determined immediately after each FPI or sham. Mean (SEM) latency time (sec) for Morris water maze performance (MWM) was assessed after trauma days 11-18. Animals were not intoxicated for MWM testing. Assessors were blinded to animal group assignment. Results were compared between groups with analysis of variance.

Results-CR and NCR animals intoxicated when injured demonstrated prolonged escape, righting, and corneal RRT after each FPI compared with non-intoxicated injured animals and non-injured shams. All injured animals demonstrated similar MWM deficits on days 11-16 compared with non-injured controls. On the last two MWM testing days, NCR animals intoxicated when injured were improved compared with $\mathrm{CR}$ intoxicated when injured (day 17, NCR $=18(1) v$ $\mathrm{CR}=32(4)$ sec; day $18, \mathrm{NCR}=17(3) v$ $\mathrm{CR}=41$ (4) sec; $\mathrm{p} \leqslant .05$ ).

Conclusions-Despite profound initial deficits, NCR animals began to learn the MWM by the last two testing days. CR animals intoxicated when injured never learned the maze.

\section{Posters: Accident Prevention}

\section{The prevention of fire deaths}

A J Hudson, J P Wyatt, T Squires, D Beard, A Busuttil

Accident and Emergency Department, Royal Infirmary of Edinburgh, Lauriston Place, Edinburgh $E H 3$ 9YW

Aim-To investigate deaths from fires in south east Scotland, with reference to possible preventative factors.

Methods-All those who died in fires in Lothian and Borders regions of south east Scotland between 1982 and 1997 (inclusive) were identified from fire service records and registrar general data. The reports of investigations into the circumstances surrounding all fatal fires were analysed, together with relevant data contained within hospital case notes, forensic medicine necropsy records, and the regional toxicological database.

Results-245 people (139 males, 106 females) died after fires during the study period. Thirty four $(14 \%)$ of those who died were children aged less than 16 years, of whom 23 were aged less than 5 years. Only 61 individuals $(25 \%)$ reached hospital alive. A total of 214 deaths $(87 \%)$ followed fires within the home, four $(2 \%)$ in the workplace, $27(11 \%)$ occurred elsewhere. Investigation of the fire scene by the fire service implicated several factors as being responsible for the majority of fires: smoking material, 82 fatalities (33\%); inflammable material placed too near heating appliances, $42(17 \%)$; “chip pans", 29 (12\%); electric faults, $20(8 \%)$; road accidents, $11(5 \%)$; children playing with matches/petrol, nine (4\%); other, 52 (21\%). Smoke detectors raised the alarm in only four cases. Sixty six individuals $(27 \%)$ had a postmortem blood alcohol concentration of more than 100 $\mathrm{mg} / \mathrm{dl}$, with 45 having concentrations exceed-

Table 10

\begin{tabular}{ll}
\hline Risk factor & Increased incidence of adverse events (95\% CI) \\
\hline Dive beyond their recommended depth & $32 \%(20 \%$ to $44 \%)$ \\
Regularly dive beyond the NDL & $37 \%(25 \%$ to $49 \%)$ \\
Do not formulate a diving plan for every dive & $12 \%(0.4 \%$ to $23 \%)$
\end{tabular}

Do not formulate a diving plan for every dive $12 \%(0.4 \%$ to $23 \%)$

$\mathrm{CI}=$ confidence interval.

ing $200 \mathrm{mg} / \mathrm{dl}$. Eighty six individuals (35\%) had a burnt area of less than $20 \%$ body surface area. Eighty nine individuals (36\%) had carboxyhaemoglobin levels of greater than $40 \%$ at the time of either hospital presentation or death.

Conclusions - The high incidence of deaths in the pre-hospital setting highlights the importance of accident prevention. Alcohol was implicated in several respects: causing dangerous behaviour, hampering recognition of the fire, and impairing escape. Safe disposal of cigarettes within the home deserves emphasis. The use of serviceable smoke detectors needs to be encouraged.

Prevention of road accident deaths in children

C Walker, J P Wyatt, A J Hudson, D Beard, A Busuttil

Accident and Emergency Department, Royal Infirmary of Edinburgh, Lauriston Place, Edinburgh EH3 9YW

Aim-To study road accident deaths in children in south east Scotland to determine the best approaches to prevent such deaths.

Methods-Deaths among children aged less than 16 years in Lothian and Borders regions during 1985-97 were identified from the registrar general and procurator fiscal. The circumstances culminating in each fatal accident were studied using detailed reports of Lothian and Borders Police Investigation Branch. Details of injuries sustained, together with pre-hospital and hospital treatment provided, were obtained form various sources, including: ambulance and hospital records and the Scottish Trauma Audit Group. Injuries were scored according to the abbreviated injury scale, 1990 revision, from which injury severity scores (ISS) were calculated.

Results-84 children (50 boys, 34 girls) died after road accidents, representing $49 \%$ of all traumatic paediatric deaths during the 13 years studied. The road accident deaths comprised 40 pedestrians $(48 \%), 32$ car passengers $(38 \%)$, and 12 cyclists (14\%). Accident analysis revealed that hazardous actions of the children themselves were responsible for most of the pedestrian accidents and all of the cycling accidents. Twenty three of the 32 car passenger deaths appeared to have resulted from mistakes or hazardous manoeuvres made by the driver of the child's car, seven resulted from actions of other drivers and in two cases, "fault" could not be attributed. Fifty four children (64\%) died before reaching hospital: 48 of these were dead when found. ISS ranged from 16 to 75, with 22 children having injuries acknowledged to be unsurvivable.

Conclusions - The greatest potential to prevent deaths from road accidents in children lies with injury prevention. To be most effective, preventative measures need to take into account the fact that actions of child pedestrians and cyclists may be hard to predict. Behaviour of both child and adult road users needs to be targeted with this in mind.
Are recreational scuba divers engaging in unsafe diving practices?

D S Vickery, C Malin, R Khodabocus, P A Evans

Department of Accident and Emergency Medicine, Leicester Royal Infirmary NHS Trust, Leicester LE1 5WW

Background-As increasing numbers of people are taking up recreational scuba diving, morbidity and mortality figures for the sport are rising. Accident and emergency departments are often providing the initial management for these patients. Adverse events while scuba diving may have serious medical complications and be implicated in fatalities. Certain aspects of diving practice may put an individual at increased risk.

Design-A questionnaire based survey of scuba divers recording all adverse diving events and associated risk factors. Undertaken at the largest inland diving centre in the UK over a two month period.

Main outcome measures-Incidence of all adverse diving events and their association with the practice of diving beyond the recommended depth for the individual's training; diving beyond the no-decompression limit (NDL); and failure to formulate a diving plan for every dive.

Results-598 divers with a $57 \%$ incidence of adverse diving events (including equipment failure, panic, and medical complications). Results are shown in table 10.

Conclusions-Adverse events are very common and occur significantly more frequently to divers who dive beyond the recommended depth to which they are trained; dive beyond the NDL; and fail to formulate a diving plan for every dive. The results of this survey have important implications in the prevention of diving accidents.

CRASH project (Co-ordinated Research into Accidents on Salford's Highways)

A Baxendale, D W Yates, J Hadfield, S Cooper, S Wright

University Department of Emergency Medicine, Clinical Sciences Building, Hope Hospital, Salford M6 $8 H D$

Introduction-Salford has a particularly high rate of road accidents involving children. In response to the "Health of the Nation" target, which aims to reduce the death rate of children under 15 years in trauma related accidents by a third, the CRASH project was set up in September 1995. The multidisciplinary steering group involved in the project consists of delegates from the universities of Manchester and Salford, Hope Hospital, Salford City Council, Greater Manchester Transportation Unit, and the Department of Transport. Preliminary investigations of accident and emergency (A\&E) and police data revealed that neither police or hospital records taken alone, gave a complete picture of all accidents involving children. Therefore to obtain the true number of such accidents, it was deemed necessary for a system of data linkage to be established. This could then be 
used to identify accident causation and consequences and to monitor the effectiveness of interventions.

Method-(1) Police and A\&E department records on all child traffic accident occurring within Salford were linked with a very high degree of accuracy to build up a common database. (2) A survey of these children was undertaken by questionnaire and telephone interview to their parents to assess their disability after two weeks and at six months. (3) Census data were investigated to consider demographic, socioeconomic, and other factors associated with the accident.

Findings $-28 \%$ of all accidents are not reported to the police, $37 \%$ do not attend the $\mathrm{A} \& \mathrm{E}$ department, although $4.4 \%$ of these are recorded as serious by the police who attend the scene of the accident. Altogether $16.8 \%$ of children coded as slightly injured in police reports were found to have serious injuries at the A\&E department. The demographic groups of females, 0-4 year olds, and car passengers had the greatest proportion of unreported accidents to the police, with the respective percentages of $30.0 \%, 42.2 \%$, and $14.4 \%$. As well as showing that children suffer substantial physical disability after a road traffic accident, the questionnaire also identified emotional trauma. At two weeks after the accident $65 \%$ of respondents to the questionnaire reported that their child had become nervous or upset; $35.3 \%$ of children were suffering from nightmares at two weeks and $22.2 \%$ were still experiencing these symptoms at six months.

Conclusions-Data provided by the police and used by the city council to make decisions on road policy are incomplete. Almost one third of incidents are not included on their database. Analysis of the more accurate CRASH database suggests that this under-reporting is not evenly represented across all patient groups, making simple mathematical correction inappropriate. More comprehensive data collection techniques are required if local authorities are to be supplied with information which accurately reflects the problems they have a statutory duty to address.

Do seatbelts work for children: a matched pairs study

Howard M Corneli, Larry Cook, J Michael Dean

Intermountain Injury Control Research Center, Salt Lake City, UT 84113, USA

Objectives-Seatbelts are designed for adults. Studies of their efficacy in children are contradictory and subject to confounding. A matched pairs study was designed to overcome this problem.

Methods-A five year database of all motor vehicle crashes (MVCs) in the state was linked to hospital records using probabilistic methods. Crashes were selected in which at least one occupant was killed or hospitalised and at least one was a child (age $<15)$. Drivers were excluded. Matched pairs were formed of an adult and child from the same vehicle. The primary outcome was hospitalisation or death. Covariates included seatbelt use and front $v$ back seat. Analysis was by matched pairs logistic regression.

Results-Overall 480 pairs were analysed. The mean (SD) age of children was 7.4 (4.9) years; of adults, 29 (15) years. Belt use was similar in children and adults $(49 \% v 48 \%)$. Backseat position was higher for children $(77 \% v 28 \%)$. Per cent killed was similar $(7 \% v 9 \%, p=0.3)$ but per cent killed or hospitalised was lower in
Table 11

\begin{tabular}{lllll}
\hline & $\begin{array}{l}\text { Overall improvement } \\
\text { in confidence }\end{array}$ & $\begin{array}{l}\text { Formal } \\
\text { teaching of skill }\end{array}$ & $\begin{array}{l}\text { Improved confidence } \\
\text { (teaching) }\end{array}$ & $\begin{array}{l}\text { Independent } \\
\text { performance of skill }\end{array}$ \\
\hline Defibrillation (\%) & 44 & 86 & 49 & 61 \\
Lead a cardiac arrest (\%) & 49 & 75 & 55 & 56 \\
Trauma primary survey (\%) & 71 & 74 & 75 & 88 \\
Tube thoracostomy (\%) & 23 & 83 & 34 & 33 \\
Shoulder reduction (\%) & 60 & 51 & 67 & 51 \\
\hline
\end{tabular}

children (13\% v 30\%, odds ratio (OR) 2.91, $95 \%$ confidence interval (CI) 2.06 to 4.11 ). Adults were at higher risk in backseat pairs (OR 3.0, 95\% CI 1.28 to 7.06 ), pairs where both were belted (OR 3.8, 95\% CI 1.89 to 7.63), and unbelted pairs (OR $2.56,95 \%$ CI 1.44 to 4.57 ). Multivariate analysis given belt use and seat position showed adults remained at increased risk of death or hospitalisation (OR $2.73,95 \%$ CI 1.7 to 4.38 ).

Conclusion-Children fare better than adults in MVCs even when confounding is eliminated. Research into protective schemes must take this innate difference into account or risk overestimating the protection offered children.

The epidemiology of paediatric autopedestrian crashes: a population based study

Howard M Corneli, Kristen L Carroll, J Michael Dean

Departments of Pediatrics, Orthopedics, University of Utah School of Medicine, National EMSC Data Analysis Resource Center, Salt Lake City, UT, USA

Objectives-To describe the epidemiology of paediatric auto-pedestrian crashes (PAPCs) in a single urban county using population based data.

Methods-Data were drawn from a four year database (1992-5) of all motor vehicle crashes on public roads in the state, and selected to represent only pedestrian victims $<21$ years of age in a large urban county. Statistical analysis was done using log linear methods.

Results-Selection yielded 1079 PAPC victims. Median age was 12 , mean age 11 ; the modal age group was 12-16 years. Month of occurrence showed peaks in March to June and in September to October. Day of week showed peaks on Monday and Friday (17\% of PAPCS each) with a nadir on Sunday (6\%). Time of day peaked at $1500-1800$ with a smaller peak at $0700-0900$. Of 20 possible pedestrian actions three (crossing not at intersection, intersection without signal, and "other in road") accounted for $48 \%$ of PAPCs. Playing in road (4\%) and running from behind vehicles $(6 \%)$ were uncommon. Police injury codes were $5 \%$ none, $17 \%$ possible, $48 \%$ moderate, $26 \%$ major, and $3 \%$ fatal. School months (September to May) were significantly over-represented among children over 5 years of age. PAPCS in non-daylight hours represented $21 \%$ of patients $0-5$ years, $12 \%$ (6-10 years), $30 \%$ (11-15 years), and $46 \%(16-20$ years) $(p<0.00001)$. Multivariate analysis revealed that death was associated with dark hours and with school months. The odds ratio for fatal outcome after dark was 7.1 (95\% confidence interval 3.5 to 19.3 ). Death, injury, and hospital admission did not vary with age category.

Conclusions-This population based study challenges some common perceptions and could help target prevention efforts.

\section{Posters: Training}

Performing practical procedures-the value of accident and emergency E Brazil, A MacNamara, G G Bodiwala Accident and Emergency Department, Leicester Royal Infirmary, Leicester LE1 5WW

Objective-To compare the confidence of senior house officers (SHOs) at performing practical medical procedures before and after working in an accident and emergency (A\&E) department and relate this confidence to extent of formal teaching and independent performance of these skills.

Method-Structured questionnaire sent to all SHOs completing an A\&E post in the Trent region in August 1997. Basic information about the respondent and the department in which they worked was requested. Inquiry was made of the subjective confidence of the respondents in performing the following procedures before and after working in A\&E: use of a defibrillator, team leading a cardiac arrest, performing a primary survey in a trauma patient, insertion of a chest drain, and reducing a dislocated shoulder.

Results-84 replies from 120 questionnaires were obtained giving a response rate of $70 \%$. The main findings are shown in table 11 .

Conclusions-The expressed confidence of SHOs in performing each of the procedures dramatically improved after working in A\&E. Formal teaching demonstrated further benefit. A significant number of SHOs however, do not perform these skills despite being confident to do so.

Learning styles of emergency medicine residents: implications for curriculum delivery

Walter C Robey III, Raffi Terzian, David Emmerling, Theodore Whitley

East Carolina University School Of Medicine, Greenville NC, Department of Emergency Medicine, Academic Support Counseling Center and Office of Medical Education, USA

Objectives-The purpose of the study was to describe the learning styles of a group of emergency medicine residents and test the feasibility of adapting the delivery of the curriculum to the residents' learning preferences.

Methods-Thirty emergency medicine resident physicians from our emergency medicine residency program were asked to complete the Canfield learning styles inventory. The learning style preference for each resident was determined and the results for the group were compiled. were returned. There were 10 respondents in each level of training (PGY1-PGY3). Twenty four $(80 \%)$ of the respondents were male and six $(20 \%)$ were female. Sixteen residents $(53 \%)$ expressed a preference for applied learning either independently or through interaction with others. Independent
Results-All 30 (100\%) of the questionnaires 
learners, who prefer to work alone in a self directed and/or self paced situation, comprised $17 \%(n=5)$ of the respondents. The remaining residents were distributed across the other types.

Conclusions-Data indicated that the greatest number of respondents fell into the applied or combination (social/applied or independent/ applied) categories, although there was considerable variability in learning styles. Applied learners, whether or not they have strong preference for either social or independent approaches, may learn more effectively through "hands-on" skills labs, workshops, simulated patient encounters, mock codes, and case scenarios. These results indicate the need to provide practical "handson" instruction with enough flexibility to allow some learners to direct their pace and course of study. Matching curriculum delivery to mixed learner typology is a challenge. Identifying the learning style preference of each resident, however, may guide curriculum delivery, enhance learning, and ultimately improve academic and clinical performance.

\section{Characteristics of long term survivors in} academic emergency medicine

Harold Thomas, N Clay Mann, John Moorhead

Oregon Health Sciences University/Portland Veterans Affairs Medical Center, USA

Objectives-Compare work hours, salary, and burnout among academic emergency medicine physicians and private practitioners with $>20$ years' experience.

Methods-Survey of practices and survival strategies among 1343 physicians identified as members of the American College of Emergency Physicians for $>20$ years.

Results-Of 813 (61\%) surveys returned, 647 worked $>80$ hours/month and were included in the analysis. The sample was divided into two groups: (1) 82 academicians reporting $>20$ hours of teaching/month and (2) 565 private practitioners.

The mean work hours/month (total: academics 203.9, private 166.5)-academics: 98.5 clinical, 50.6 administrative, 46.8 teaching, 8 other and private: 126 clinical, 34.3 administrative, 2.2 teaching, 3 other.

Annual income-academics: $\quad<\$ 100 \mathrm{~K}$ $4.6 \%$; $150 \mathrm{~K}, 13.9 \%$; $200 \mathrm{~K}, 33.7 \%$; $250 \mathrm{~K}$ $27.9 \%$; $>250 \mathrm{~K}, 19.7 \%$ and private: $<\$ 100 \mathrm{~K}$ $6.0 \%$; $150 \mathrm{~K}, 18.1 \%$; 200K, $26.1 \%$; $250 \mathrm{~K}$ $25.3 \%$; >250K, $23.5 \%$.

Reported feelings of "burn-out"academics: none, $36.5 \%$; little, $36.5 \%$; somewhat, $18.2 \%$; much, $2.4 \%$ and private: none $26.4 \%$; little, $37.2 \%$; somewhat, $23.3 \%$; a lot 9.4\%; much, $3.5 \%$.

Reported years of remaining employmentacademics: $>15$ years, $3.7 \%, 10$ years, $24.7 \%$ 5 years, $54.3 \%$; $<5$ years, $17.3 \%$ and private $>15$ years, $3.5 \%$; 10 years, $18.2 \%$; 5 years $46 \%$; $<5$ years, $32.2 \%$

Conclusions-Academic emergency physicians work significantly more total hours than their peers in private practice $(\mathrm{p}<0.001)$, but are similar in degree of burn-out. These "extra" hours are in the area of teaching. Altogether $70 \%$ of emergency physicians practising for $>20$ years plan to quit clinical medicine within the next 10 years.
Characteristics of women emergency physicians

Erica Frank, Vicken Y Totten, Louise Andrew Catholic Medical Center of Brooklyn and Queens, NY, Department of Medicine, Emory University School of Medicine, Atlanta, GA, Center for Professional Well-Being, Durham, NY 27705, USA

Background/objectives - We examined the differences and similarities between the personal and professional characteristics of women emergency physicians (WEPs) compared with other women physicians (OWPs).

Methods - This study compared a subpopulation of the Women Physicians' Health Study (WPHS) database against all other respondents. WPHS was a mail survey sent to a stratified random sample of women physicians graduating between 1950 and 1989 .

Results - Of the 4501 respondents to the WPHS, 90 were WEPs, representing $2.5 \%$ of the (weighted) sample. WEPs were younger than OWPs, were more likely to be single or be part of an unmarried couple, and less likely to be married. WEPs' personal health habits did not differ significantly from OWPs. Young WEPs were more likely than young OWPs to be board certified in another specialty and less likely to be board certified in their primary specialty. WEPs are more likely than OWPs to practise in a rural area WEPs work less and earn more than OWPs. Although WEPs were more likely to report high work stress, they felt a similar level of professional satisfaction and specialty commitment as OWPs. WEPs considered health practices counselling to be less relevant to their practices than did OWPs, and felt less well prepared by their training to counsel patients.

Conclusions-With some interesting exceptions, WEPs resemble OWPs. The emergency department encounter may represent a teachable moment which is missed because (at least women) emergency physicians feel such counselling is either irrelevant, or that they are inadequately trained to provide it.

\section{Posters: Medical Emergencies}

\section{Malaria in inner London}

P Leman, N Mir

Accident and Emergency Department, St Thomas' Hospital, Lambeth Palace Road, London SE1 7EH

Objective-To describe the malarial cases treated in an inner London hospital during a single year.

Methods-Retrospective analysis of clinical records of all patients with a positive malaria film during 1996 treated at University Hospital Lewisham.

Results -44 case records were available for review; 40 cases $(90.9 \%)$ were due to Plasmodium falciparum. Thirty seven patients were admitted to hospital; there were no deaths. Only five patients (11.3\%) had taken adequate malarial prophylaxis. A total of 119 inpatient bed days were utilised in the treatment of malaria in one year. In $75(63 \%)$ of these bed days observation was the only reason for continued inpatient treatment. The commonest laboratory findings are mild increase of serum bilirubin to a mean of 28 $\mu \mathrm{mol} / 1$ (95\% confidence interval (Cl) 23.3 to 32.7 ), and a low platelet count to a mean of $124 \times 10^{12} / 1(95 \% \mathrm{Cl} 103.6$ to 144.3$)$. A bilirubin greater than $20 \mu \mathrm{mol} / 1$ was found in $72.9 \%$ of patients ( $95 \%$ CI $58.7 \%$ to $87.3 \%$ ).
The platelet count was less than $150 \times 10^{12} / 1$ in $77.3 \%$ of patients $(95 \% \mathrm{Cl} 64.9 \%$ to $89.7 \%$ ).

Conclusions-Malaria is not uncommon in inner London. While most cases are admitted, few complications are usually seen. Many cases may well be able to be treated with a brief hospital admission, possibly to an accident and emergency observation ward.

Two years' experience of outpatient treatment of deep vein thrombosis from the accident and emergency department

D F O'Shaugnessy, S Akbar*, V M O’Neill ${ }^{\star}$, C Tovey ${ }^{\star}, \mathrm{H}$ LDraper ${ }^{\star}$, A C L Miller

${ }^{*}$ Departments of Accident and Emergency and Haematology, St Peters Hospital NHS Trust, Chertsey, Surrey

This study confirms the safety and effectiveness of the outpatient treatment of acute deep vein thrombosis (DVT) initiated in the accident and emergency (A\&E) department. A total of 1088 patients with suspected DVT were referred to the $A \& E$ department by their general practitioners. They were then assessed by $A \& E$ staff and 738 patients were discharged after light reflection rheography (LRR). This investigations is used as a screening test because it has a high negative predictive value for DVT and our study supports its use to determine which of the referred patients can safely be discharged without further investigation for DVT. Altogether 352 patients had a positive result on LRR and subsequently underwent venography; 162 patients had positive venograms, of whom 159 have now been treated with low molecular weight heparin and warfarin, as outpatients. No patients had further embolic episodes. Two had minor bleeding (while on warfarin); none had major bleeding. The cost of this treatment compares favourably with the cost of continuous intravenous unfractionated heparin therapy delivered by syringe pump, which requires daily monitoring of activated partial thromboplastin time. Direct liaison with community nurses has minimised the impact on general practitioner workload. A total of 1272 hospital bed days were saved during this period (an estimated saving of $£ 320000$ ) by outpatient treatment of patients with DVT. In addition to the financial saving, this study clearly demonstrates that the times of year when the hospital bed days were saved coincide with the periods of greatest demand in UK hospitals.

Brain attack. How good is the early management of subarachnoid haemorrhage in accident and emergency?

$S$ Thomson, J Ryan, J Lyndon

Accident and Emergency Department, Royal Sussex County Hospital, Brighton, East Sussex

Objectives - To examine the speed of (1) presentation, (2) diagnosis, and (3) referral of patients with subarachnoid haemorrhage who ultimately require neurosurgical care. To find which clinical features most commonly result in the correct diagnosis and what proportion are treated appropriately.

Design-Four neurosurgical centres provided lists of subarachnoid haemorrhage referrals for 1997. The medical notes and computed tomograms from 20 accident and emergency (A\&E) departments within the South Thames region were reviewed.

Subjects-Records of 105 patients were obtained. The mean age was 53 , similar to multicentre studies. Female to male ratio was 
2.4: 1. Median World Federation of Neurosurgical Societies subarachnoid grade was 2 .

Outcome measures-(1) Timings of onset of symptoms, presentation, computed tomography, and referral. (2) Proportion referred directly from $A \& E$ to neurosurgery. (3) Clinical features most commonly resulting in the correct diagnosis. (4) Number given nimodipine, intravenous fluids, and analgesia. Results $-65 \%$ of patients presented to A\&E within six hours, $78 \%$ within three days. Computed tomography was performed on $47 \%$ within four hours, $60 \%$ within eight, and $87 \%$ within 24 hours of arrival. Altogether $84 \%$ reached the neurosurgical centre within three days of presentation, $56 \%$ within three days of the onset of symptoms. Thirty six per cent were referred to a neurosurgical centre directly from $A \& E$, of these $13 \%$ had nimodipine, $25 \%$ intravenous fluids, and $38 \%$ analgesia before transfer. Symptoms occurred with the following frequencies: headache $80 \%$, neck stiffness $34 \%$, photophobia $28 \%$, motor deficit $22 \%$, and warning headaches $11 \%$.

Conclusions-Subarachnoid haemorrhage is managed suboptimally. A protocol will be introduced based on guidelines produced by the Society of British Neurosurgeons. Further studies of its effectiveness will be carried out in due course.

Thrombolytic therapy for stroke: a review of recent studies and controversies

Tiffany N Osborn, Marlan P LaMonte, Wade Gaasch

University of Maryland, USA

Objectives-Review major multicentre trials of thrombolytic therapy for ischaemic stroke and discuss the use in stroke treatment.

Methods-Thrombolytic agents reviewed were tested in at least two prospective, double blind, multicentre, randomised controlled studies. A minium of 100 patients must be confirmed as having ischaemic stroke before randomisation. Reviewed trials: NINDS, ECASS, MAST-I, MAST-E, and ASK. Discussion parameters include agent used, dose, ancillary medication, intracranial haemorrhages, mortality, and neurological outcomes. Results-ASK, MAST-E, MAST-I, were stopped early due to increased mortality. NINDS showed using a dose of $0.9 \mathrm{mg} / \mathrm{kg}$ within three hours of stroke onset yields significant increase in complete patient recovery or minimal deficit at three months (Barthe index 95-100: t-PA $50 \%$, placebo $38 \%$ ) NIHSS of $0-1$ : t-PA $31 \%$, placebo $20 \%$. Modified Rankin 0-1: t-PA 39\%, placebo $26 \%$ ). ECASS demonstrated the importance of strict adherence to administration guidelines. The 90 day mortality of the intention-totreat (ITT) group (with protocol violations) $(p=0.04)$ was: treated $22 \%$ and placebo $15.6 \%$ compared with 90 day mortality of $19.4 \%$ and $14.8 \%$ respectively in the treatment population (TP) (without protocol violations) $(\mathrm{p}=0.17)$ Both the ITT and TP groups were predefined variables. In the TP group (without protocol violations) no significant difference in mortality was demonstrated and the numbers reported were similar to NINDS outcomes. The computed tomography protocol violations of severe early infarct showed a $40 \%$ mortality in ECASS (treated $48.4 \%$, placebo $28.6 \%$ ).

Conclusions-NINDS showed t-PA to significantly improve neurological outcome of stroke without significant increase in mortality. However, this is a dangerous drug and any institution wishing to use t-PA for ischaemic stroke should strictly adhere to protocol guidelines for optimal results.

Heart rate variability analysis: a valuable adjunct in the emergency departmen diagnosis of acute myocardial infarction Brian A Nester ${ }^{\star}$, William C Dalsey*, Una Geary $^{\star}$, Georges Ramalanjaona ${ }^{\star \star}$, Carol Ter-

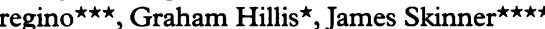
*Albert Einstein Medical Center, Philadelphia, PA, ** Newark Beth Israel Hospital, Newark, Nf, ${ }^{* *}$ Cooper Hospital University Medical Center, Camden, NF, $\star * \star \star$ Delaware Water Gap Science Institute, Bangor, PA, USA

Study objective-To investigate the utility of heart rate variability (HRV) as a screening tool for the emergency department diagnosis of acute myocardial infarction (AMI) in high risk chest pain patients.

Methods-This is a prospective, ongoing, multicentred, blinded, observational study. A validated clinical algorithm was used to identify a convenience sample of high risk chest pain patients ( $>7 \%$ chance of AMI). Patients were enrolled via informed consent and underwen 20 minutes of continuous electrocardiographic (ECG) recording on a bedside HRV unit in the emergency department. These results were withheld from emergency department clinicians. Non-linear HRV measures (PD2i) were calculated by an investigator blinded to patient outcomes. The primary outcome measure was AMI, as diagnosed by World Health Organisation criteria. Emergency department diagnosis was based upon the contemporaneous combination of: emergency department physician clinical impression, serial ECG tracings, and initial CK/CK-MB measures.

Results-HRV was recorded in 160 patients, 34 of whom subsequently had a discharge diagnosis of AMI. Only 18 of these AMIs were recognised by emergency department physicians (sensitivity of emergency departmen diagnosis $=53 \%$ ). A critical PD2i measurement $(\leqslant 1.6)$ was present in 19 of the 34 AMI patients (sensitivity $=56 \%$ ). By combining HRV criteria with standard emergency department diagnostic criteria, we would have successfully identified 30 of the 34 AMI patients. This combination represents an $88 \%$ sensitivity for diagnosing AMI in the emergency department.

Conclusions - HRV is at least as sensitive as current methods used to diagnose AMI in the emergency department. This simple, noninvasive measure, if included in the immediate evaluation of chest pain patients, could markedly improve the sensitivity of the emergency department diagnosis of AMI.

Depressed heart rate variability is predictive of ventricular arrhythmia in emergency department chest pain patients Brian A Nester ${ }^{\star}$, Una Geary ${ }^{\star}$, Carol Terregino ${ }^{\star \star}$, Georges Ramalanjaona $a^{\star \star \star}$ Graham Hillis`, William C Dalsey ${ }^{\star}$, James Skinner ${ }^{\star \star \star \star \star}$

*Albert Einstein Medical Center, Philadelphia PA, ${ }^{*}$ Cooper Hospital University Medical Center, Camden, Nf, ***Newark Beth Israel Hospital, Newark, Nf, $\star * \star \star$ Delaware Water Gap Science Institute, Bangor, $P A, U S A$

Study objective-To determine the reliability of heart rate variability (HRV) as a screening tool for high risk emergency department chest pain patients in the prediction of ventricular arrhythmia during hospital admission.

Methods-This is a prospective, ongoing, multicentred, blinded, observational study. A validated clinical algorithm was used to identify a convenience sample of high risk chest pain patients $(>7 \%$ chance of acute myocardial infarction (AMI)). Patients were enrolled via informed consent and underwent 20 minutes of continuous electrocardiographic recording on a bedside HRV unit at emergency department presentation. All patients underwent in-house cardiac monitoring. Non-linear HRV measures (PD2i) were calculated by an investigator blinded to patient outcomes. The primary outcome measure was the occurrence of ventricular arrhythmia, which was determined from review of telemetry records for each patient after hospital discharge or death

Results-Thirteen of 158 patients developed ventricular arrhythmia (that is ventricula fibrillation or ventricular tachycardia of more than five beats) during hospital admission Eight of these patients had AMI. Mean PD2 scores were significantly lower in patients who developed ventricular arrhythmia, than those who did not develop arrhythmia (1.3 $v 1.8, \mathrm{p}$ $<0.05)$. A cut off value of PD2i less than or equal to 1.2 , which has been associated with increased risk of VF in high risk patients with non-sustained ventricular tachycardia, identified all four patients who suffered cardiac arrest, two of whom died.

Conclusions-HRV is a non-invasive clinical measure, applicable in the emergency department setting, which identifies chest pain patients at high risk for the development of life threatening cardiac arrhythmias. Although further studies are needed to quantify the effects of coexisting clinical factors which influence this novel measure, our initial results suggest that HRV shows promise as a prognostic tool in the emergency department assessment of chest pain patients who may be at risk for ventricular arrhythmia.

Intravenous magnesium is ineffective in severe adult asthmatics

Robert S Porter, Brian A Nester, Una Geary, William C Dalsey

Albert Einstein Medical Center, Philadelphia, PA, USA

Introduction-Intravenous magnesium sulphate $\left(\mathrm{MgSO}_{4}\right)$ has been tried in the emergency department treatment of asthma since the mid-1980s, but published reports vary as to its efficacy. The literature suggests that it may be effective in the more severely ill asthmatic. We evaluated intravenous $\mathrm{MgSO}_{4}$ in adult asthmatics having a moderate to severe exacerbation.

Subjects and methods-Study population was a convenience sample of adult asthmatics between the ages of 18-55 presenting to the emergency department with a peak expiratory flow (PEF) of $<100 \mathrm{1} / \mathrm{min}$ or $<25 \%$ of predicted. Patients received either $2.0 \mathrm{~g}$ $\mathrm{MgSO}_{4}$ or placebo in a randomised, double blind fashion shortly after arrival in the emergency department. All patients received standard asthma therapy, including inhaled bronchodilators and intravenous steroids. Outcome variables were: improvement in PEF compared with baseline, subjective respiratory distress as measured by the Borg dyspnoea scale (BDS), and need for admission.

Results-55 patients completed the study protocol, 12 of whom were repeat visits, leaving 43 for analysis, $19 \mathrm{MgSO}_{4}$ and 24 placebo. There was no statistically significan difference in the starting PEF or BDS. PEF 
improved by a mean of $127 \mathrm{l} / \mathrm{min}$ in the $\mathrm{MgSO}_{4}$ group and $162 \mathrm{l} / \mathrm{min}$ placebo, difference $-35 \mathrm{l} / \mathrm{min}$ ( $95 \%$ confidence interval (CI) -96 to 25 ). Final median BDS was 1.0 in the $\mathrm{MgSO}_{4}$ and 0.5 in the placebo group (lower = better), Mann-Whitney $\mathrm{p}=0.128$. Hodges-Lehmann estimate of shift parameter of BDS was $=1.0$ favouring placebo (95\% CI 0.0 to 2.0). Admission: 6/19 (31.6\%) patients on $\mathrm{MgSO}_{4}$ were admitted $v$ $5 / 24(20.8 \%)$ on placebo, Fisher's exact test $\mathrm{p}=0.325$, difference $10.8 \%$ (95\% CI $44.0 \%$ to $-19.7 \%$ ).

Conclusions-2.0 $\mathrm{g}$ of $\mathrm{MgSO}_{4}$ intravenously did not decrease the admission rate of a group of severe adult asthmatics. A clinically significant improvement in PEF and BDS was ruled out.

\section{A clinical pneumonia pathway decreases} time to antibiotic administration

Anthony Magalski, Eric Williams, Ronald S Benenson, Sally Cavanaugh

York Hospital/Pennsylvania State University Emergency Medicine Residency, USA

Objectives-In April, 1994, York Hospital implemented a clinical pathway for patients with an admission diagnosis of pneumonia. The pathway was initiated after a 1992-93 study found a longer length of stay and higher mortality rate for York Hospital patients when compared with a "benchmark". The current study was undertaken to determine if implementation of a clinical pathway would result in decreased time to administration of antibiotics, decreased length of stay and decreased mortality as intended, both in the initial year after pathway implementation and two years later.

Methods-A retrospective chart review was completed for patients entered in the pneumonia pathway via the emergency department. Three cohorts of pneumonia patients were compared: (1) three months immediately before pathway implementation (1/94 to 3/94), (2) 9-12 months after implementation of the pathway (1/95 to 3/95), and (3) 33-36 months after implementation of the pathway ( $1 / 97$ to $3 / 97)$. Four standard antibiotic regimens were used: community acquired, community acquired penicillin allergic, nursing home acquired, and nursing home acquired penicillin allergic. Demographics, medical history, presentation, signs and symptoms, and outcome data were abstracted from each patient's medical record.

Results - The time to antibiotic administration decreased from 315 minutes prepathway to 174 minutes postpathway which was preserved over time $(p<0.0001)$. The percentage of patients who received antibiotics in the emergency department increased from $58 \%$ to $93 \%$, again persisting over time ( $p$ $<0.0001)$. Mortality decreased from $9.5 \%$ to $4.9 \%$ ( $p=0.41)$. Length of stay decreased from 9.7 to 6.7 days $(p=0.008)$.

Conclusion-The use of a pneumonia clinical pathway significantly reduced time to antibiotic administration and increased the percentage of patients receiving antibiotics in the emergency department. Mortality decreased, substantially, but was not statistically significant due to low incidence. Length of stay also decreased over this period of time, but may have been affected by a number of factors.
A randomised, double blind comparison of intravenous magnesium sulphate and intravenous prochlorperazine in the treatment of acute headache

Steven R Ginder, Brenda K Oatman, Marc L Pollack

York Hospital/Pennsylvania State University Emergency Medicine Residency, USA

Objective-Previous uncontrolled, nonrandomised trials suggest that magnesium sulphate $\left(\mathrm{MgSO}_{4}\right)$ is effective in treatment of headache. The objective of this study was to determine the efficacy of intravenous MgS04 versus prochlorperazine (PRO) in the treatment of acute headache.

Design-Prospective, randomised, double blind trial.

Setting-55000 visit community teaching hospital emergency department.

Participants-Patients aged 18-50, presenting to the emergency department with chief complaint of headache, excluding pregnant females, patients with known adverse reaction to the study drugs, and patients with serious medical disease.

Intervention-Patients rated their pain on a visual analogue scale before and 30 minutes after study drug infusion. Each was randomly assigned either $2 \mathrm{~g} \mathrm{MgSO}_{4}$ or $10 \mathrm{mg}$ PRO intravenously.

Results - 36 patients were enrolled; 20 received PRO, 16 received $\mathrm{MgSO}_{4}$. The groups were similar with regard to gender, duration of headache, and medications taken before emergency department presentation. There was complete or partial pain relief in $90 \%$ $(18 / 20)$ of the PRO group and $56 \%(9 / 16)$ of the $\mathrm{MgSO}_{4}$ group. The difference was statistically significant using $\chi^{2}$ analysis $(p<0.04)$. Mean pain reduction, as evaluated by the visual analogue scale, was $4.8 \mathrm{~cm}$ for PRO and $2.4 \mathrm{~cm}$ for $\mathrm{MgSO}_{4}$ by $t$ test $(\mathrm{p}<0.05)$ with $95 \%$ confidence interval 0.056 to 4.694). PRO caused fewer adverse effects $(\mathrm{p}<0.05)$.

Conclusions-Intravenous PRO is highly effective in treatment of headache and significantly better than intravenous $\mathrm{MgSO}_{4}$, with fewer adverse effects.

Nocturnal asthmatics show evidence of inflammation induced decoupling of mitochondrial oxidative metabolism

Monica Kraft, Gregory R Bell, James M Walther, Juno Pak, Richard J Martin, Brian T Williams, Charles B Cairns

National Fewish Medical and Research Center, Colorado Emergency Medicine Research Center, University of Colorado Health Sciences Center, Denver, Colorado, USA

Introduction-We have shown that nocturnal asthma is associated with increased airway inflammation with influx of macrophages. Macrophage induced inflammatory mediators, such as tumour necrosis factor- $\alpha$, can alter mitochondrial oxidative phosphorylation. Hypothesis-Nocturnal exacerbations asthma result in decoupling of peripheral tissue cellular oxidation (cytochrome $\mathrm{a}_{3} \mathrm{a}_{3}$ redox state) from oxygen delivery (tissue saturation). Methods-Tissue saturation and the cytochrome $\mathrm{a}_{\mathrm{a}} \mathrm{a}_{3}$ redox state were continuously monitored from $15: 00$ to $18: 00$ and from 23:00 to $06: 00$ in 11 patients: five patients with nocturnal asthma (NA), defined as $>15 \%$ fall in overnight peak expiratory flow rate (PEFR) over 4/7 nights, two patients with nonnocturnal asthma (NNA), defined as a fall in PEFR $<10 \%$, and four non-asthmatic controls (CO) using near infrared spectroscopy of the

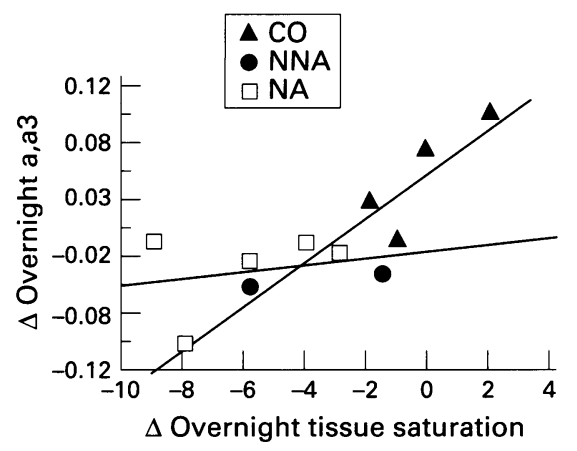

Figure 1

left deltoid region. Tissue saturation quantitates the ratio of oxyhaemoglobin and oxymyoglobin to deoxyhaemoglobin and deoxymyoglobin in the tissue monitored. Spirometry was performed at 16:00 and 03:00. Results-The per cent overnight fall in forced expiatory volume in one second was $-26.3 \pm 4.4 \%$ in NA, $-6.2 \pm 2.2 \%$ in the NNA group, and $0.4 \pm 2.3 \%$ in the $\mathrm{CO}$ group. In contrast to CO and NNA $(r=0.85 ; p=0.03)$, the fall in cytochrome a, $a_{3}$ redox state did not correlate with the change in tissue saturation in the NA group ( $r=0.39 ; \mathrm{p}=0.56$; see fig 1 ). This mitochondrial oxidation is consistent with decoupling of electron flow.

Conclusions-The impairment of lung function in nocturnal asthma is associated with decoupling of the tissue mitochondrial cytochrome $a, a_{3}$ redox state from oxygen delivery. This decoupling may be due to macrophage influx at night with accompanying inflammation in patients with nocturnal asthma.

Factors influencing delay in presentation of patients with acute ischaemic stroke to an urban hospital

Michael Washington, Michael Woods, Sharita Warfield, Edna Gordon

Howard University Hospital, Washington, DC, USA

Objective-To identify factors associated with a delay in presentation to the hospital of patients experiencing symptoms of acute stroke. The recent advances and time limitations in thrombolytic therapy for acute stroke have prompted the need to identify the reasons that patients delay in presenting to the emergency department.

Methods-This is an observational prospective survey study done in the emergency department of an urban level 1 trauma centre with 40000 visits. Persons presenting to the emergency department with an acute neurological deficit were surveyed between 1 October 1998 to 1 January 1998 . The survey identifies presenting symptoms, symptom onset time, time the decision was made to go to the hospital, time of arrival to triage, reasons for delay, patient impression of problem, patient awareness of new stroke treatment.

Results - 37 patients participated with ages ranging from 38-98 years old; 20 female and 17 male. Average delay was 13.7 hours (range $35 \mathrm{~min}$ to 80 hours). Seven of 37 or $19 \%$ thought about stroke ( $81 \%$ did not); $4 / 37$ or $11 \%$ knew of new treatment ( $89 \%$ did not) Conclusion-These results suggest a need for an educational program about symptoms and treatment for acute stroke targeted for urban settings. 
Table 12

\begin{tabular}{lllllll}
\hline Measured & & Baseline & 1 & 2 & 3 & 4 \\
\hline Heliox & PEFR & 193 & 255 & 283 & 241 & 201 \\
Oxygen & PEFR & 195 & 243 & 251 & 248 & 211 \\
Heliox & FEV $_{1}$ & 1.4 & 1.7 & 1.9 & 1.7 & 1.5 \\
Oxygen & FEV $_{1}$ & 1.2 & 1.5 & 1.5 & 1.6 & 1.4 \\
Heliox & FEF & 80 & 107 & $115^{\star}$ & 93 & 70 \\
Oxygen & FEF & 69 & 87 & $83^{\star}$ & 85 & 73 \\
No heliox/oxygen & & $94 / 105$ & $94 / 105$ & $69 / 77$ & $27 / 38$ & $9 / 8$ \\
\hline
\end{tabular}

${ }^{\star} \mathrm{p}<0.01$, all other pairs and analysis are not significant.

Use of heliox versus oxygen driven nebulisation of albuterol in the treatment of asthma

Jack Horowitz, Robert E O'Connor, Vinay Nadhkarni, Patricia Resnick, Peter Grossweiler Department of Emergency Medicine, Medical Center of Delaware, Christiana Care Health System, Newark, Delaware, USA

Objectives-Heliox has been successfully used in severe brochospasm. We conducted this study to compare airway response to oxygen versus heliox powered nebuliser.

Methods-This prospective randomised blinded trial was conducted at the emergency department of a tertiary care teaching hospital. Patients with mild to moderate asthma were eligible. Patients received up to four (at the discretion of the treating physician) $2.5 \mathrm{mg}$ doses of albuterol via nebulisation using either gas. Physical examination, peak expiratory flow rate (PEFR), forced expiratory volume in one second (FEV), forced expiratory flow $25 \%-75 \%$ (FEF), pulse oximetry, and disposition were measured by a respiratory therapist at baseline, and after each treatment up to four. Data were analysed using $\chi^{2}, t$ test, Pearson's $r$, and repeated measures analysis of variance.

Results-The results are shown in table 12 .

There were no significant differences in the physical examination, pulse oximetry, and admission rate. There was a high degree of correlation between PEFR: FEV $(0.87)$,

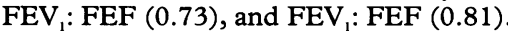

Conclusions-PEFR, $\mathrm{FEV}_{1}$, and FEF highly correlate. For mild to moderate asthma, heliox and oxygen appear equivalent, and use of the more expensive heliox appears unwarranted Whether continuous treatment with heliox between nebulisations eases the work of breathing, remains to be determined.

The NINDS rt-PA Stroke Study: sustained benefit at one year

T G Kwiatkowski, R Libman , M Frankel, B

Tilley, L Morganstern, M Lu, J Broderick, J

Marler, T Bron, and the NINS rt-PA Stroke Study Group

Background-The NINDS rt-PA Stroke Study showed a significant benefit at three months for patients with acute ischaemic stroke treated with rt-PA.

Objective-We hypothesised that benefit would be sustainied at six and 12 months. Methods-Patients in the NINDS rt-PA Stroke Study were contacted by telephone at six and 12 months by a researcher blinded to their treatment allocation for their status (alive or dead), their ability to perform daily activities (Barthel index), and assessment of functional disability (modified Rankin and Glasgow outcome scales). As in the primary paper, favourable outcome was assessed using a global odds ratios, a summary statistic invorporating the proportion on patients with minimal or no disability on the three scales at six and 12 months.

Results - The results are shown in table 13. Conclusion-Patients treated with rt-PA were at least $30 \%$ more likely than placebo treated patients to have minimal or no disability at six and 12 months consistent with the findings reported at three months.

\section{Posters: Resuscitation}

Stress in accident and emergency staff during cardiopulmonary resuscitation in the presence of relatives

Russell Boyd, Sandra White

Department of Emergency Medicine, Hope Hospital, Stott Lane, Salford M6 6HD

Objectives-Firstly, to ascertain if the presence of a patients' relatives during cardiopulmonary resuscitation altered perceived stress in accident and emergency (A\&E) staff and, secondly to ascertain if the outcome of resuscitation altered perceived stress in said staff.

Methods-An anonymous objective structured questionaire was administered within a 24 hour period to all attending A\&E staff at sequential adult non-traumatic cardiopulmonary resuscitation attempts at two centres with a "supported" relatives policy for attendance at resuscitation. The questionaire was based on objective criteria for an ICD-10 "acute stress reaction".

Results- $89 \%$ of staff surveyed completed the questionaire; $22 \%$ of all staff involved had two or more symptoms of an ICD-10 "acute stress reaction". There was no difference in reported

\begin{tabular}{|c|c|c|c|c|c|}
\hline & \multicolumn{2}{|c|}{$\%$ Favourable outcome } & \multirow[b]{2}{*}{$\begin{array}{l}\text { Odds ratio } \\
(95 \% \text { CI) }\end{array}$} & \multirow[b]{2}{*}{$\begin{array}{l}\text { Relative risk } \\
(95 \% \text { CI })\end{array}$} & \multirow[b]{2}{*}{ p Value } \\
\hline & $\begin{array}{l}t-P A \\
(n=312)\end{array}$ & $\begin{array}{l}\text { Placebo } \\
(n=312)\end{array}$ & & & \\
\hline \multicolumn{6}{|l|}{ Six months } \\
\hline Global test & - & - & $1.7(1.3$ to 2.3$)$ & - & 0.0006 \\
\hline Barthel & 50 & 37 & $1.7(1.2$ to 2.4$)$ & $1.4(1.1$ to 1.6$)$ & 0.001 \\
\hline Modified Rankin & 41 & 29 & $1.8(1.3$ to 2.5$)$ & $1.4(1.2$ to 1.8$)$ & 0.001 \\
\hline Glasgow & 43 & 31 & $1.6(1.2$ to 2.3$)$ & $1.3(1.1$ to 1.7$)$ & 0.0041 \\
\hline \multicolumn{6}{|l|}{12 months } \\
\hline Global test & - & - & $1.7(1.2$ to 2.3$)$ & - & 0.0012 \\
\hline Barthel & 50 & 38 & $1.6(1.1$ to 2.1$)$ & $1.3(1.1$ to 1.5$)$ & 0.005 \\
\hline Modified Rankin & 41 & 28 & $1.8(1.3$ to 2.5$)$ & $1.5(1.2$ to 1.3$)$ & 0.001 \\
\hline Glasgow & 43 & 32 & $1.5(1.1$ to 2.2$)$ & $1.3(1.1$ to 1.6$)$ & 0.006 \\
\hline
\end{tabular}

incidence of stress reactions with or without relatives presence. There was a $50 \%$ incidence of stress reaction with a positive outcome to resuscitation compared with a $19 \%$ incidence with a negative outcome to resuscitation. This was a non-significant trend which did not reach statistical significance.

Conclusions-The presence of relatives at cardiopulmonary resuscitation does not alter perceived stress in A\&E staff. Successful resuscitation is associated with a nonsignificant trend to an increase in perceived stress.

Directed postischaemic substrate infusion reverses the impairment of oxidative phosphorylation after cardiac ischaemia and reperfusion injury

Charles B Cairns, Anthony A Ferroggiaro, James $M$ Walther, Jeffrey Peterson, Alden $\mathrm{H}$ Harken, Anirban Banerjee

Colorado Emergency Medicine Research Center, University of Colorado Health Sciences Center, Denver, Colorado, USA

Background-Ischaemia and reperfusion can lead to inhibition of key metabolic enzymes and impaired myocardial mechanical function. We hypothesised that identification of the postischaemic lesion in oxidative phosphorylation permits the selection of substrates to restore function during reperfusion.

Methods-Isolated rat hearts were subjected to global ischaemia $\left(25 \mathrm{~min}, 37^{\circ} \mathrm{C}\right)$ and reperfusion (40 $\mathrm{min})$. Left ventricular developed pressure (LVDP) and the cytochrome a, $\mathrm{a}_{3}$ redox state (near infrared spectroscopy) were continuously monitored. Oxygen consumption was assessed for the NADH (mitochondrial complex I) and $\mathrm{FADH}_{2}$ (complex II) pathways, in both the resting and maximal ADP stimulated states. Myocellular ox phos capacity was measured using an assay specific for mitochondrial ATPase. Substrate dosing was optimised for electron flow at cytochrome $\mathrm{a}, \mathrm{a}_{3}$. Thus, the hearts were randomised to either succinate $(200 \mu \mathrm{mol} / \mathrm{l})$, acetyl-Lcarnitine $(200 \mu \mathrm{mol} / \mathrm{l})$ or control for the first 5 min of reperfusion.

Results-Ischaemia and reperfusion in the control group resulted in an impairment of NADH (complex I) ox phos capacity $(1.4 \pm 0.4$ $v$ control $3.9 \pm 0.6 \mathrm{nmolATP} / \mathrm{min} / \mathrm{mg} ;{ }^{\star} \mathrm{p}<.05 v$ control) and depressed LVDP $(49 \pm 3 \%$ of baseline $\left.^{\star}\right)$. The ox phos capacity for the succinate utilising $\mathrm{FADH}_{2}$ pathway remained intact $(2.6 \pm 0.3 v 2.4 \pm 0.4)$. There was enhanced LVDP recovery after ischaemia and reperfusion with both succinate $(89 \pm 8 \%$ of baseline $\left.{ }^{\star}\right)$ and acetyl-L-carnitine $(74 \pm 7 \%$ of baseline $\left.{ }^{\star}\right)$. Diminished transport resulted in depletion of electrons from cytochrome a, $a_{3}$ during both ischaemia and early reperfusion, which was reversed by providing either succinate or acetyl-L-carnitine as substrate.

Conclusions-Ischaemia and reperfusion results in a defect at mitochondrial complex I, but not complex II. Directed substrate infusion restores redox state balance and cardiac function after ischaemia.

Clinical experience with the rapid four step technique for emergency cricothyrotomy

Bo Tomas Brofeldt, John C Sakles, Mary Osborn, Edward A Panacek

University of California, Davis Medical Center, USA

Background-The rapid four step technique (RFST) is a new method of cricothyrotomy 
specifically designed by emergency physicians to be faster and easier than standard techniques.

Objective-To describe our initial clinical experience with this technique.

Design-Prospective, consecutive case series at a large academic institution.

Intervention-A brief questionnaire was completed by the operator for each cricothyrotomy performed using the RFST, between 7/95 and 11/97.

\section{Statistics-Descriptive.}

Results-A total of 24 cricothyrotomies were performed using the RFST. Twenty one had unsuccessful attempts at endotracheal intubation before RFST; $100 \%$ of RFST attempts successfully established an airway, $88 \%$ on the first attempt. The estimated time for RFST was less than one minute for all cases. It was the first cricothyrotomy outside a laboratory setting for $71 \%$ of operators. Seventy five were performed pre-hospital and $25 \%$ in the emergency department: $37 \%$ by emergency medicine residents and $63 \%$ by flight nurses. The skin and soft tissue were incised to identify the anatomy before performing the stab incision in $50 \%$ of patients. The stab incision was enlarged to pass the tube in $63 \%$ of patients. Nine long term survivors were without complications from RFST, and 11 available necropsy records confirmed correct placement without complications. All operators with previous cricothyrotomy experience rated the RFST as being easier than their previous technique. Conclusion-Initial clinical experience with the RFST indicates that it is safe, rapid, effective, and preferred by emergency personnel.

Oxygen delivery and utilisation during moderate hypoxia and hyperoxia in adult volunteers

Jeffrey Peterson, Gregory R Bell, Brian T Williams, David Nyman, Paul Bender, Anirban Banerjee, Charles B Cairns

Colorado Emergency Medicine Research Center, University of Colorado Health Sciences Center, Denver, Colorado, USA

Background-Hypoxia can induce a number of complex respiratory and circulatory adaptations, including hyperventilation and vasodilatation, which could influence end organ oxygen delivery and utilisation.

Objective-To directly assess tissue oxygen delivery and utilisation during hypoxia and hyperoxia.

Methods-Healthy adult volunteers underwent graded hypoxia to $65-70 \%$ oxygen saturation as monitored by arterial pulse oximetry, followed by reoxygenation with $100 \%$ inspired oxygen. Near infrared spectroscopy was used to continuously monitor tissue oxygen delivery (oxyhaemoglobin; $\mathrm{Hbo}_{2}$ ), availability (oxygen saturation; $\mathrm{Sto}_{2}$ ), and utilisation (mitochondrial cytochrome $\mathrm{a}_{3} \mathrm{a}_{3}$ redox state) in the deltoid region. Serum acetoacetate and $\beta$ hydroxybutarate were measured after baseline, hypoxia, and hyperoxia.

Results - 22 subjects were enrolled in the study (nine women). With hypoxia, Sto ${ }_{2}(\Delta-13.3 \pm$ $1.2 \%$; ${ }^{\star} \mathrm{p}<.05 v$ baseline values $), \mathrm{HbO}_{2}(\Delta-7.7$ $\pm 1.1 \%$ baseline $\left.{ }^{\star}\right)$, and the cytochrome a, $\mathrm{a}_{3}$ redox state $\left(\Delta-3.8 \pm 1.9 \%\right.$ baseline $\left.{ }^{\star}\right)$ all fell from baseline room air values. With hyperoxia, Sto $_{2}(\Delta+15.8 \pm 1.6 \%), \mathrm{Hbo}_{2}(\Delta \pm 9.5 \pm 1.1 \%$ baseline $\left.{ }^{\star}\right)$, and the cytochrome $\mathrm{a}, \mathrm{a}_{3}$ redox state $\left(\Delta+10.21 \pm 1.8 \%\right.$ baseline $\left.^{\star}\right)$ all rose from the hypoxic values. While the a, $a_{3}$ redox state correlated with $\mathrm{HbO}_{2}$ during hypoxia ( $r=0.60$; $\mathrm{p}<0.05$ ), the cytochrome $\mathrm{a}, \mathrm{a}_{3}$ redox state was relatively more oxidised after $100 \%$ oxygen.

Table 14

\begin{tabular}{lllll}
\hline & $E-C P R$ & $S A A P$ & ROSC 15 min & ROSC 60 min \\
\hline Spinal cord (\%) & 8 & 96 & 253 & 91 \\
Brainstem (\%) & 10 & 145 & 240 & 68 \\
Basal ganglia (\%) & 6 & 50 & 356 & 85 \\
Cortex (\%) & 6 & 24 & 255 & 66 \\
Myocardium (\%) & 13 & 248 & 232 & 139 \\
\hline
\end{tabular}

$\mathrm{n}=9$.

There were no significant changes in serum acetoacetate and $\beta$ hydroxybutarate levels with hypoxia and hyperoxia.

Conclusions-(1) During acute hypoxia, peripheral tissue oxygenation delivery an utilisation fall quickly and dramatically. (2) During reoxygenation with $100 \%$, peripheral oxygen delivery is quickly restored to baseline values while the cytochrome $a, a_{3}$ redox state becomes relatively hyperoxidised. (3) In healthy adults, hyperoxia after hypoxia rapidly depletes electrons from the respiratory chain, resulting in a relatively oxidised state within the mitochondria, despite no evidence of overall changes in the cellular oxidative state.

Race and socioeconomic status do not predict adverse outcome of out-ofhospital cardiac arrest

Anthony J Sayegh ${ }^{\star}$, Kevin $\mathrm{H} \mathrm{Chu}^{\star}$, Raymond Jackson ${ }^{\star}$, Robert A Swor ${ }^{\star}$, Josh Gitlin ${ }^{\star}$, Robert M Domeier ${ }^{\star \star}$, Eliezer Basse ${ }^{\star \star \star}$, Dena Smith $\star \star \star \star$, William Fales ${ }^{\star \star \star \star}$

*William Beaumont Hospital, Royal Oak, Michigan, **St foseph's Mercy Hospital, Ann Arbor, Michigan, ${ }^{\star *}$ North Oakland Medical Center, Pontiac, Michigan, $\star * \star \star$ Michigan State University/Kalamazoo Center for Medical Studies, Kalamazoo, Michigan, USA

Objective-To assess whether socioeconomic status or race is associated with adverse outcome after an out-of-hospital cardiac arrest (OOHCA)

Methods-A convenience sample of OOHCA of presumed cardiac origin from seven suburban cities in Michigan, 1991 to 1996 . Median household income (HHI), utilising patient home address and 1990 census tract data, was dichotomised above and below 1990 state median income. Patient race was dichotomised as black or white. Outcome was defined as survival to hospital discharge. Multiple logistic regression and Pearson's $\chi^{2}$ were used for analysis.

Results-Of 1321 cases with complete data for analysis, the average age was $67.3 \pm 16.0,939$ $(71.1 \%)$ were white, $587(44.4 \%)$ arrests were witnessed, and $65(4.9 \%)$ were discharged alive. There was no significant difference between races with respect to witnessed arrests, VT/VF arrest rhythms, and the emergency medical service response interval. Whites were more likely to be above median HHI (57.1\% v 26.2\%, p<0.001). Adjusted odds ratios for predictors of survival were witnessed arrest (odds ratio $=3.76,95 \%$ confidence interval (CI) 1.7 to 8.2 ), VT/VF (odds ratio $=8.74,95 \% \mathrm{CI} 3.7$ to 10.8 ), but not race (odds ratio $=0.68,95 \%$ CI 0.3 to 1.4 ), or socioeconomic status (odds ratio $=1.51,95 \%$ CI 0.8 to 2.8 )

Table 15

\begin{tabular}{lllllll}
\hline & Pre-I & 1 min & 4 min & 7 min & 10 min & 16 min \\
\hline Blood loss $(\mathrm{ml} / \mathrm{kg})$ & $0(0)$ & $3(1)$ & $11(3)$ & $16(3)$ & $20(4)$ & $24(5)$ \\
Cardiac output $(1 / \mathrm{min} / 30 \mathrm{~kg})$ & $3.2(0.7)$ & $2.1(0.8)$ & $1.3(0.50)$ & $9(0.5)$ & $0.6(0.3)$ & $0.4(0.3)$ \\
Mean aortic pressure $(\mathrm{mm} \mathrm{Hg})$ & $84(12)$ & $69(15)$ & $43(14)$ & $37(15)$ & $30(11)$ & $26(14)$ \\
\hline
\end{tabular}

${ }^{\star} \mathrm{p}<0.05 v$ control, Fisher's exact test; $\# \mathrm{p}=0.05 v$ control, $t$ test.
Conclusion-In this population, neither race nor socioeconomic status was independently associated with a worse with a worse outcome after OOHCA.

Cerebral perfusion with selective aortic arch perfusion during cardiopulmonary resuscitation

James E Manning

Department of Emergency Medicine, School of Medicine, University of North Carolina at Chapel Hill, USA

Objective-Selective aortic arch perfusion (SAAP) is an invasive perfusion therapy for cardiac arrest using a descending aortic arch balloon catheter to selectively perfusion the heart and brain. Regional blood flow (RBF) to the brain was assessed in a canine model of ventricular fibrillation.

Methods-After 10 min of cardiac arrest and 2 min of external cardiopulmonary resuscitation (E-CPR), SAAP with oxygenated autologous blood was used until return of spontaneous circulation (ROSC). Coloured microspheres were used to assess myocardial and cerebral blood flow during prearrest, E-CPR, SAAP, $15 \mathrm{~min}$ of ROSC, and $60 \mathrm{~min}$ of ROSC. RBF to cerebral cortex, basal ganglia, brainstem, spinal cord, and heart were assessed.

Results-All nine animals achieved ROSC. Table 14 shows mean RBF (\% of prearrest) for the various brain areas and the myocardium.

Conclusion-SAAP is an effective invasive artificial perfusion technique during cardiac arrest. Perfusion to the brain, especially the basal ganglia and cortex, was less than to the heart during SAAP despite subsequent hyperaemia. Limited cerebral cortical perfusion during SAAP may significantly affect neurologic recovery. The data raise questions about cerebrovascular responses to global hypoperfusion and the canine model being used.

Selective aortic arch perfusion to treat profound haemorrhagic shock: preliminary work using a swine model of multiple liver lacerations

James E Manning, O John Ma, D Neil Batson, Thomas R Griggs, Christopher C Baker Department of Emergency Medicine, School of Medicine, University of North Carolina at Chapel Hill, USA

Objective-Selective aortic arch perfusion (SAAP) uses an aortic arch balloon catheter to occlude the thoracic aorta and perfuse the heart and brain. In haemorrhagic shock due to abdominal or pelvic trauma, SAAP may restore vital organ perfusion while limiting 
further haemorrhage. We studied the effects of SAAP in a liver trauma model.

Methods-Eight anaesthetised instrumented swine, $23-40 \mathrm{~kg}$, had wires placed through each of four liver lobes such that when pulled they resulted in lacerations of about $50 \%$ of the thickness of the lobes. Rate and volume of blood loss were assessed by a suction system. When a plateau in blood loss and aortic pressure was reached, animals received either rapid intravenous saline infusion alone or SAAP with oxygenated shed autologous blood with aortic occlusion.

Results-Rate of blood loss, volume of blood loss, aortic pressure, and response to saline versus SAAP autologous blood are shown in table 15. Return of spontaneous circulation and cardiopulmonary resuscitation diastolic coronary perfusion pressure were greater in the SAAP group.

Conclusion-This preliminary work suggests that SAAP may be a useful therapy for profound haemorrhagic shock due to bleeding distal to the thoracic aorta. Further study of SAAP in haemorrhage models is nee

Lidocaine use during intubation: is it used for intracranial pressure prophylaxis?

Christopher F Richards ${ }^{\star}$, Erik D Barton*, Robert J Vissers ${ }^{\star \star}$, Ron M Walls ${ }^{\star}$, on behalf of the NEAR investigators

${ }^{\star}$ Brigham and Women's Hospital, Department of Emergency Medicine, Harvard Medical School, ${ }^{\star}$ UNC, Chapel Hill, Department of Emergency Medicine, USA

Background -Intravenous lidocaine blunts the increase in intracranial pressure (ICP) during laryngoscopy and intubation and is widely recommended.

Objective-To describe the use of lidocaine use during intubation of medical and trauma patients.

Methods-Prospective, descriptive study using a convenience sample of patients intubated between 8/96 and 8/97 at 11 emergency departments participating in the National Emergency Airway Registry (NEAR). Data collected included indication for intubation, drugs administered, and reasons cited for choice of intubation method. More than one indication or reason may have been listed for each patient. $Z$ test for proportions was used to analyse the data.

Results-Of 1288 intubations reported in the registry, 1277 listed an indication. The primary indication was traumatic in 439 and medical in 833. Potentially raised ICP was a reason for the method of intubation in 154 patients. Lidocaine use was reported for $17.0 \%$ of medical and $42.6 \%$ of trauma intubations $(p=0.0,95 \%$ confidence interval (CI) of difference $20 \%$ to $31 \%$ ). Head trauma was an indication for 217 patients, $55.3 \%$ of whom received lidocaine $(p=0.003,95 \%$ CI of difference $4.6 \%$ to $20 \%$ ). Lidocaine use was reported in $66.8 \%$ cases where possible increased ICP was a reason for the method of intubation performed, while in $28.4 \%$ of cases it was not $(p=0.0,95 \% \mathrm{CI}$ of difference $31 \%$ to $46 \%$ ).

Conclusions - In the emergency departments participating in NEAR, lidocaine is used more often in patients with head trauma or potentially increased ICP, but not universally. Future outcomes data may prove or disprove its utility.
Confirmation of endotracheal tube placement-analysis of 1288 emergency department intubations

Ron M Walls ${ }^{\star}$, Mark J Sagarin ${ }^{\star}$, John C Sak-

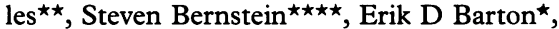
Richard E Wolfe ${ }^{\star}$, on behalf of the NEAR investigators

* Brigham and Women's Hospital, Department of Emergency Medicine, Harvard Medical School, **UNC, Chapel Hill, Department of Emergency Medicine, ${ }^{\star \star} U C$ Davis Medical Center, Department of Emergency Medicine, $* * * *$ St LukesRoosevelt Medical Center, USA

Objective-Physical examination methods of endotracheal tube placement confirmation have been proved to less reliable than newer methods, such as end tidal carbon dioxide detection and aspiration. This study sought to determine whether newer objective methods to confirm tracheal placement of endotracheal tubes placed in the emergency department are used to sufficient extent to be considered a standard of care.

Methods-Prospective observational study of 1288 emergency department intubations in 11 US teaching hospitals during the pilot phase (8/96 to 8/97) for the National Emergency Airway Registry (NEAR).

Results-1288 intubations were registered over the pilot period; 984 were adults, 144 children $<18$ years, 160 age unknown. Rapid sequence intubation, oral intubation with sedation only, oral intubation without medications, and nasal intubation were the first method in $80 \%, 6 \%, 14 \%, 0 \%$ of children, and $79 \%, 8 \%, 11 \%, 2 \%$ of adults. By method, detected oesophageal intubations were reported in $3 \%, 2 \%, 3 \%$, and $4 \%$ respectively. There were no cases of undetected oesophageal intubation. Most common methods used for tube placement confirmation included auscultation (95\%), end tidal carbon dioxide determination $(83 \%)$, chest radiography $(73 \%)$, and aspiration techniques $(15 \%)$.

Conclusion-In these 11 centres, physical examination is supplemented by end tidal carbon dioxide detection in over $80 \%$ of emergency department intubations to confirm tube placement. Aspiration methods are infrequently used.

\section{Posters: Audit}

Clinical critical incident monitoring in an accident and emergency department: identifying areas of risk in order to improve quality

Conor V Egleston

Emergency Department, Southampton General Hospital, Tremona Road, Southampton SO16 $6 Y D$

Introduction-Emergency medicine by virtue of high patient workloads and the unpredictable nature of the work is an area where adverse patient events are likely. We established a system to provide early warning of such events with the aims of quality improvement and early warning of litigation and complaints.

Methods - We introduced a system of critical incident monitoring and asked all members of staff to contribute by completing a critical incident form and returning it to the critical incident coordinators in the department. The coordinator then investigated the incident and took any appropriate action.

Results-There were 65 incidents reported in the first six months of the system's operation. Twenty six reports involved critical care situa- tions, five incidents related to child protection protocols, six incidents involved pre-hospital care, eight incidents reported the department's resources being stretched by workload. There were a range of other clinical conditions cited as incidents. The commonest results of the incidents were near misses ( 37 incidents), injuries (11 incidents), dissatisfaction or breakdowns in communication (12 incidents), and temporary physiological change (three incidents). The commonest environmental contributing factors were workload (19 incidents) and lack of space (four incidents). The most common contributing human factors were failure to follow guidelines (18 incidents), lack of knowledge (13 incidents), inattention (10 incidents), and lack of supervision (five incidents).

Conclusion-The system allowed us to identify factors which contributed to incidents and areas where guidelines or protocols were inadequate. It also helped us in our communication with other agencies and specialties allowing us to work out protocols between us. In addition we developed a potential early warning system for complaints and litigation against the department.

An audit of the effective clinical use of the accident and emergency review clinic $M$ Hayder Hassan, V Sethu Kumar Accident and Emergency Department, Medway NHS Trust, Windmill Road, Gillingham, Kent ME7 5NY

The aim of this study was to analyse the value and clinical effectiveness of the review clinic service based within the Medway accident and emergency (A\&E) department. It analysed the reasons for, and the appropriateness of, referral of patients to the review clinic by the A\&E medical staff, following the patient's initial visit to the department for treatment.

Review clinics have tended to provide a "safety net" for patients passing through any major A\&E department.

A retrospective study was undertaken of 512 patients, who were referred to the review clinic during January and February 1998. This was agreed so as to enable comparison of two groups of $A \& E$ junior medical staff; the first group in their last month of A\&E experience, the second during their first month within A\&E. Of the total, 257 attendances were studied thoroughly, 62 being repeat appointments.

The indications for referral were critically reviewed with $22(8.5 \%)$ considered to be inappropriate for review clinic consultation Examination of key aspects for inappropriate referral highlighted oversight of the use of fracture clinic for orthopaedic review.

Opportunities for staff education have been recognised and an even greater effective use of the review clinic is aimed for and also a reduction in the non-attendance rate which was $13 \%$ overall for those two months. ded.

Pre-existing disease on the UK Trauma Network database-an assessment of data quality

J Majumder, G Brown, P Driscoll, D W Yates clo UK Trauma Audit and Research Network, Clinical Sciences Building, Hope Hospital, Salford

Introduction-Previous work using the UK Trauma Audit and Research Network (UK- 
TARN) database has shown that nationally, $38.8 \%$ of trauma patients had at least one preexisting medical condition (PEMC). Furthermore, the presence of PEMCs affects both mortality and length of hospital stay. However, there are potential problems in data interpretation in this study. Firstly, bias could have arisen because the PEMC status was not recorded in $61 \%$ of cases. Secondly, it is not known which sources the UKTARN data gatherers use to identify PEMC.

Aims - To quantify the importance of "missing" data and the inconsistency of data gathering methods for the UKTARN database.

Methods-From one centre, analysis of 70 consecutive casenotes with the PEMC record recorded as "missing" on the UKTARN database was undertaken. A questionnaire survey was then carried out on all $(n=90)$ UKTARN data gatherers to clarify the sources they use to identify the PEMC status of a patient

Results-Overall, 21(34.8\%) of the "missing" group had at least one PEMC. Eighty data gatherers responded to the questionnaire (response rate $=88.9 \%$ ). There was considerable inconsistency in sources used to identify PEMCs. The most frequently used source was the "past medical history" from the admission clerking (96\%). Only $62 \%$ used previous admission notes and 33\% drug history; 5\% carried out staff interviewing.

Conclusions-The variety of sources used to identify a patient's PEMC status could give rise to inconsistent results. Consequently, conclusions drawn from UKTARN database analysis regarding PEMCs should be interpreted cautiously until the effects of this variation can be determined. However, the "missing" dataset has a lower incidence of PEMC than the overall national database. This suggests that the direction of effect described by such analyses is unlikely to be affected, only the magnitude.

An analysis of 5357 admissions to an emergency department based clinical decision unit

J J Kahler, G Tokarski, R M Nowak, N Khoury, M C Tomianovich

Henry Ford Hospital, Department of Emergency Medicine, 2799 West Grand Blvd, Detroit, Michigan 48202, USA

Background-The clinical decision unit (CDU) is an extension of the emergency department. The literature reports that $10 \%$ $40 \%$ of CDU patients will require hospital admission despite ongoing and aggressive CDU care.

Objective-To determine rates of the 10 most common CDU protocols of an urban tertiary care emergency department, and how the CDU hospital admission compares with reported rates.

Methods-Retrospective review of 5357 CDU patients during 1996-97. The most commonly utilised CDU protocols were identified and hospital admission and discharge rates calculated for each. Fisher's exact test was used to determine differences in hospital admission rates.
Results -4076 patients utilised the 10 most frequent CDU protocols. Disposition data was available for 3932 (96\%). Overall, 25\% of patients required hospital admission despite CDU therapy. Individual hospital admission rates varied from $16.9 \%$ to $61.8 \%$ and are shown in table 16.

Sickle cell hospital admission rate was statistically different from all others $(p<0.01)$. Hospital admission rates were different for two distinct groups of protocols: chest pain dehydration/cellulitis/gastrointestinal bleed when compared with asthma/COPD/ pneumonia $(\mathrm{p}<0.01)$.

Conclusions-An overall CDU hospital admission rate of $25 \%$ agrees with reported series, but significant variation exists among individual protocols. The utility of CDU protocols with high hospital admission rates require further analysis with protocol alterations.

Epidemiology of US citizen deaths occurring during travel abroad in 1996

Stephen W Hargarten, James Holliman

Medical College of Wisconsin, Department of Emergency Medicine, PennState Geisineger Health System, USA

Objective-To determine the causes of death and the distribution of numbers of deaths in different countries of US citizens travelling abroad in calendar year 1996, and to compare those data with the last report on this subject from 1983.

Methods - The US State Department files of death reports on US citizens who died in another country from 1 January 1996 through 31 December 1996 were reviewed. Demographic data for each case were recorded. Cause of death was recorded as that listed on the State Department report or in more detailed accompanying death certificates. Cases were excluded as "non-travellers" (US citizens permanently or semipermanently residing in another country) if the person did not have a current US address, or had a foreign address and an expired US passport or a renewal US passport issued in the foreign country.

Results-2186 deaths were identified as probable US citizen travellers. A total of 409 deaths (19\%) occurred in Mexico; the other countries with the (sequential) greatest number if travellers deaths were Germany, UK, Philippines, Spain, France, Greece, Italy, Israel, and Japan. Thirty per cent of the deaths were due to myocardial infarction or sudden cardiac death, $7.2 \%$ due to other cardiovascular causes, $6.7 \%$ motor vehicle crashes, $5.7 \%$ other trauma, $3.1 \%$ homicide, $3.9 \%$ drowning, $4.6 \%$ strokes, $6.5 \%$ cancer, and $26 \%$ unknown, unrecorded, or "natural".

Conclusion-Cardiovascular causes remain the most common cause of death in US citizen international travellers, and risk of death from homicide, motor vehicle accidents, and drowning appears to have increased since 1983.

\section{Posters: Paediatric Emergency}

Characteristics of children with asthma presenting to a paediatric accident and emergency department

T Beattie, Judith Brown, A Duncan

Accident and Emergency Department, Royal Hospital for Sick Children, Sciennes Road, Edinburgh EH9 1 LF

Objective-To define the characteristics of children presenting to a paediatric accident and emergency (A\&E) department with asthma.

Design-Prospective, descriptive study on all children presenting with asthma, or who left the department with a diagnosis of asthma.

Subjects-366 children leaving the A\&E department with the diagnosis of asthma.

Main outcome measures-Age and sex distribution of children with asthma; frequency of diagnosis made in $A \& E$ and relation of symptoms to each characteristic. Evidence of control and effect of asthma on daily living. Results-366 children presented on 320 occasions; $62 \%$ were male with $41 \%$ children aged 2 or 3 . Twenty eight per cent of children had the diagnosis made in the A\&E department. A previous diagnosis of asthma was significantly associated with nocturnal and early morning cough and evidence of poor control. Mean time off education for the previously diagnosed group was $7 \pm 12$ days, compared with $2 \pm 8$ days for those undiagnosed ( $\mathrm{p}<0.0003) ; 50 \%$ were admitted.

Conclusions-The A\&E department has a significant role in identifying children not previously diagnosed as having asthma. Improved diagnosis will lead to better implementation of British Thoracic Society guidelines and hopefully this will lead to a better quality of life for these children.

Paediatric emergency care needs assessment in Guyana

Robby Mahadeo, Andy Jagoda

Department of Emergency Medicine, Mount Sinai Medical Center New York, NY, USA

Objectives-Guyana Watch conducts annual humanitarian missions. The goals are (1) to analyse paediatric illnesses presenting to temporary clinics in Guyana; (2) to identify areas of focus for preventive programs; and (3) to identify preparatory programs necessary for future participants.

Methods-Retrospective analysis of all patients $<20$ years seen in 13 temporary clinics, $8 / 23 / 96$ to $9 / 7 / 96$, for age, gender, illness severity (based on complaint and vital signs), and final diagnosis.

Results - 546 patients, 2 months to 20 years; $51 \%$ males and $49 \%$ females. Fifteen per cent were triaged "critical" in need of immediate interventions, for example intravenous antibiotics, fluid resuscitation, surgery, and bronchodilators. Diagnostic categories: $52 \%$ infectious diseases; $28 \%$ rashes; $3 \%$ neurological illness; $2 \%$ congenital heart disease; $15 \%$ other. A wide spectrum of illnesses were

Table 16

\begin{tabular}{|c|c|c|c|c|c|c|c|c|c|c|}
\hline & Chest pain & Dehydrated & Cellulitis & GI bleed & Asthma & Abdom pain & Pneumonia & $C H F$ & $C O P D$ & Sickle cell \\
\hline $\begin{array}{l}\text { No admitted to hospital } \\
\%\end{array}$ & $\begin{array}{l}298 \\
16.9\end{array}$ & 53 & 39 & 38 & 203 & 63 & 49 & 63 & 71 & 115 \\
\hline No discharged & $\begin{array}{l}16.9 \\
1413\end{array}$ & $\begin{array}{l}20.1 \\
198\end{array}$ & $\begin{array}{l}22.1 \\
132\end{array}$ & $\begin{array}{l}24.0 \\
114\end{array}$ & $\begin{array}{l}25.7 \\
548\end{array}$ & $\begin{array}{l}28.5 \\
150\end{array}$ & $\begin{array}{l}31.6 \\
96\end{array}$ & $\begin{array}{l}33.8 \\
115\end{array}$ & $\begin{array}{l}42.5 \\
103\end{array}$ & $\begin{array}{l}61.8 \\
65\end{array}$ \\
\hline$\%$ & 80.1 & 75.8 & 75.0 & 72.1 & 69.3 & 67.8 & 61.9 & 61.8 & 56.9 & 34.9 \\
\hline
\end{tabular}


diagnosed including meningitis, staphylococcal scalded skin syndrome, pyloric stenosis, hypertension, and child abuse.

Conclusions-(1) $80 \%$ of complaints were related to rashes or infectious diseases; future missions must emphasise preparation in caring for these entities. (2) Infectious diseases accounted for $52 \%$ of complaints, identifying an area of focus for prevention strategies in Guyana. (3) $15 \%$ of patients were "critical" suggesting limited access to, or lack of confidence in, permanent resources, supporting the need to prepare both temporary and permanent care providers in managing unstable patients.

Project HEAL: a community based, learner centred children's health project Julius Goepp, Lori Edwards, Laura Syron, John Massad

University of Rochester, USA

Objectives-Provide children's health education, screening, and evaluation services to underserved families and help them negotiate the complex health care system using community health workers (CHWs).

Methods-Project HEAL (Health, Education, and Love) uses community members to deliver health education, home safety screenings, WIC services, and health and human service referrals. Planned additions include using a World Health Organisation screening tool to evaluate children with acute minor illnesses. A learner centred model is used to respond to community needs. Since workers are involved from program planning to service delivery, data collection, and analysis, the community itself sets many of the project's priorities.

Results-After two years, Project HEAL has strong community support. The CHWs are seen as credible and trustworthy experts on health and the health care system. Case families report feeling more stable healthwise and CHWs are shown to be "cultural translators" by assisting families with the complexities of the health care system. Of the 130 families enrolled, 70 are currently active. Training for the CHWs' use of assessment tool is nearly complete. The CHWs are developing a powerful data collection system to measure progress on health status indicators and families' knowledge, attitudes, beliefs, and behaviours.

Conclusions-Community based and learner centred programs require longer startup periods than conventional programs, but are likely to yield higher levels of community support and greater access to community members. Data collection will be more acceptable and interventions better informed and thus more successful.

\section{Posters: Diagnostic Testing}

Physicians are unable to measure pulsus paradoxus by sphygmomanometry Gregory D Jay, Kazuya Onuma, Robert J Davis, Constantine Gatsonis, Anthony Mansell, Dale Steele

Departments of Emergency Medicine and Pediatrics, Brown University School of Medicine, Providence, Rhode Island, USA, Department of Molecular Medicine, Tohoku University, fapan

Accurate measurement of pulsus paradoxus (PP) is one of several measures advocated in the NHLBI asthma management guidelines-a pulsus of $>12 \mathrm{~mm} \mathrm{Hg}$ warrants hospital admission. It is the only measure that is non-effort dependent and therefore the one which could be used in children less than 5 years old. A model of induced PP in a trained healthy subject without respiratory disease was constructed with a fixed inspiratory resistance and measurement of both inspiratory and non-invasive beat-beat blood pressure. A total of 19 attending physicians from emergency medicine and critical care disciplines were assessed for ability in measuring PP by sphygmomanometry and by palpation in an idealised quiet setting. At inspiratory pressures of $-10,-15,-20$, and $-25 \mathrm{~mm} \mathrm{Hg}$, true PP (PPt) was 13.7, 16.2, 19.1, and 20.7 $\mathrm{mm} \mathrm{Hg}$ respectively $(\mathrm{F}=14.8, \mathrm{p}<0.0001$; analysis of variance). A bias plot of the physician measured PP (PPm) against the PPt demonstrated enormous variability in the PP measurement $(r=0.04 ; \mathrm{p}=0.80)$. Palpation appeared to be more effective in assessing for PP $-80 \%$ of physicians detected a PP of 16.2 $\mathrm{mm} \mathrm{Hg}$, an increase from $46 \%$ for $13.7 \mathrm{~mm}$ $\mathrm{Hg}$. The present guidelines do not account for the challenges around measuring PP, especially in tachypnoeic patient. Sphygmomanometric determination of PP should be abandoned and new aids developed through technological innovation.

Can accurate laboratory results be obtained from a functioning IV site?

Jim J Blake, C Keith Stone, Robert F Herfel, J Stephan Stapczynski

University of Kentucky, Department of Emergency Medicine, M53 800 Rose Street, Lexington, KY 40536-0298, USA

Objective-To determine if accurate laboratory results can be obtained on blood samples drawn from a functioning IV site.

Design-Experimental.

Setting: University hospital.

Participants-Ten normal volunteers.

Interventions-Traditional phlebotomy was performed and the blood was sent for the following studies: sodium, potassium, chloride, carbon dioxide, blood urea nitrogen (BUN), creatinine, glucose, ionised calcium, haemoglobin, haematocrit, white blood cells (WBC), platelets, prothrombin time (PT), partial thromboplastin time (PTT), and international normalised ratio (INR). An IV of normal saline was started in the opposite arm and allowed to run at $125 \mathrm{ml} /$ hour for 30 minutes. The IV was stopped, $5 \mathrm{ml}$ of blood was drawn from the IV and discarded. Blood was then drawn from the IV and sent for the same studies as traditional phlebotomy. Data was analysed using paired $t$ test with alpha set at 0.05 to determine statistical significance.

Results-The results are shown in table 17. Conclusion - No statistically significant laboratory values were noted except for creatinine, which was not clinically significant. Laboratory results from traditional phlebotomy or from an existing normal saline IV site appear to yield clinically similar results.

Comparison of laboratory and ABG determinations of potassium from intraosseous samples

Roque P Ruggero, Carl Hsu

Beth Israel Medical Center, New York, USA

Use of intraosseous aspirates on an ABG analyser has not been evaluated in the medical literature. Point-of-care testing with the ABG analyser may provide clinicians the extra minutes necessary to resuscitate the critically ill paediatric patient.

Objectives-To determine whether an ABG analyser can run intraosseous samples and whether a significant difference exists between results from the ABG $v$ laboratory.

Methods-Bone marrow aspirates were obtained with Jamshidi intraosseous needles from two sedated and paralysed pigs. From each intraosseous needle, $2 \mathrm{ml}$ were aspirated into a syringe and transferred to red top tubes with separators for analysis by the laboratory, and $1 \mathrm{ml}$ was aspirated directly into a heparinised syringe for immediate analysis on a Gem Premier ABG. Six pairs of samples were obtained from one pig and five from the other. The laboratory $v$ ABG values for the samples were analysed with a $t$ test for paired samples.

Results-For this small pilot study $(n=11$ paired sets of samples), the mean difference between each two samples was determined. Using a paired $t$ test, the mean difference was $0.12 \mathrm{mmol} / \mathrm{l}$ with a standard deviation of 0.90 and $95 \%$ confidence interval of -0.725 to 0.486 . The intraosseous material did not adversely affect the ABG analyser cartridge.

Discussion-These animal data suggest that intraosseous samples will not compromise the ability of the ABG analyser to determine potassium values. Furthermore, there was no statistically significant difference between intraosseous sample results obtained on the Gem Premier analyser and those determined by the laboratory. A larger sample size is necessary to determine if a clinically significant difference exists.

Table 17

\begin{tabular}{lllll}
\hline & Phlebotomy & IV site & Mean difference & p Value \\
\hline Sodium & 139.6 & 139.8 & 0.2 & 0.51 \\
Potassium & 3.96 & 3.88 & 0.08 & 0.27 \\
Chloride & 107.6 & 107.9 & 0.3 & 0.19 \\
Carbon dioxide & 25.3 & 25.0 & 0.3 & 0.43 \\
BUN & 11.7 & 11.6 & 0.1 & 0.68 \\
Creatinine & 0.97 & 0.93 & 0.04 & $0.04^{\star}$ \\
Glucose & 130.5 & 131.1 & 0.6 & 0.37 \\
Ionised calcium & 2.19 & 2.17 & 0.02 & 0.14 \\
Haemoglobin & 13.85 & 13.81 & 0.04 & 0.69 \\
Haematocrit & 41.12 & 40.73 & 0.39 & 0.19 \\
WBC & 10.07 & 10.07 & 0 & 1.0 \\
Platelets & 190 & 187.7 & 2.3 & 0.63 \\
PT & 12.08 & 12.06 & 0.02 & 0.68 \\
PTT & 27.65 & 27.21 & 0.44 & 0.43 \\
INR & 0.962 & 0.963 & 0.001 & 0.89 \\
\hline
\end{tabular}

$\star$ Significant $\mathrm{p}<0.05$. 
Seroprevalence of varicella antibodies among new house officers

K Alagappan ${ }^{\star}, \mathrm{L} \mathrm{Fu} u^{\star}, \mathrm{S}$ Strater ${ }^{\star}, \mathrm{V}$ Casker ${ }^{\star}$, C Auerbach $\star \star$

${ }^{*}$ Department of Emergency Medicine, Long Island fewish Medical Center, NY, **Wurzweiler School of Social Work, Yeshiva University, New York, USA

Background -Varicella, as an illness common to children, can occur in non-immune susceptible adults. In adults varicella can cause serious morbidity and mortality. House officers with non-protective titres are at risk for contracting and spreading the diseases to patients they serve. There is also significant loss in work time and wages. In August of 1996, the Centers for Disease Control recommended that non-immune health care providers be vaccinated with the varicella vaccine (VV).

Objective-Document seroprevalence of varicella antibodies among house officers starting their residency.

Setting-University affiliated teaching hospital.

Methods-Serology testing on house officers beginning residency in July 1997. Subjects provided information regarding demographics, PMH, previous varicella exposure, and prior administration of VV. Serum was tested using the FIAX Test Kit (Bio-Whitaker). Statistical methods included the Student's $t$ test and $\chi^{2}$.

Results-154 house officers participated. The median age was 28 (range 24-50 years). History of varicella infection was given by $76 \%(117 / 154)$ of the subjects, $10 \%(16 / 154)$ reported no history of infection, and $14 \%(21 /$ 154) were uncertain. Seven per cent (11/154) of the participants had received VV previously. Overall, $4 \%(6 / 154)$ had non-protective titres to varicella. Of the 117 house officers who reported a history of varicella, only one had non-protective titres $(0.9 \%)$; of those who reported no history of varicella, $32 \%(5 / 16)$, had non-protective titres, $\mathrm{p}<0.001$. Of the house officers who received VV in the past, $18 \%(2 / 11)$ had non-protective titres.

Conclusion-Reported history of varicella or the administration of $\mathrm{VV}$ does not assure presence of protective titres. This underscores the need for house officers to be tested for varicella and not accept history of infection or administration of $\mathrm{VV}$ as a determinant of protection.

\section{Posters: Trauma}

\section{A five year study of high falls in Edin- burgh}

J P Beale, J P Wyatt, D Beard, C A Graham Department of Accident and Emergency Medicine, Royal Infirmary of Edinburgh

Aim-To investigate high falls in a defined urban population, including pre-hospital deaths, hospital deaths, and survivors.

Methods-Hospital presentations after a "high fall" (free fall $>2 \mathrm{~m}$ ) during five years (1993-7) were studied using prospectively collected data from the Scottish Trauma Audit Group. Entry criteria and data collection followed that of the Major Trauma Outcome Study. Revised trauma scores were calculated from physiological data at presentation and injury severity scores (ISS) calculated from the abbreviated injury scale, 1990 revision. TRISS methodology enabled probabilities of survival to be derived using the UK coefficient. The circumstances preceding high falls in all those who died were obtained from detailed police investigation reports. Injuries sustained by those who died were extracted from necropsy reports and scored to produce an ISS.

Results-During the five years, high falls accounted for $6.0 \%$ of all patients on the hospital's trauma database. Of 340 high falls, $61(18 \%)$ died at the scene, $26(8 \%)$ died in hospital, and $253(74 \%)$ survived to hospital discharge. Injuries after high falls accounted for 3563 days in hospital beds, including 281 days in intensive care. The height of fall was known in 323 cases: 235 fell between 2 and 10 m ( 28 died); 88 fell $>10 \mathrm{~m}$ ( 55 died). Fifty four $(62 \%)$ of the 87 deaths appeared to be suicides.

Overall, 187 individuals had an ISS $<15$ (two died); 129 had ISS of 15-66 (61 died); 24 had ISS of 75 (all died). TRISS methodology yielded seven unexpected survivors and three unexpected deaths.

Conclusions-High falls account for a significant number of deaths each year and take a considerable toll on hospital resources. The potential to reduce the death rate by improving hospital treatment appears limited. The greatest potential lies with injury prevention measures, but since the majority of deaths were suicides, the way forward in this respect remains unclear.

The epidemiology of surfboard related injuries

M Gillett, $S$ W Goodacre

24 Brookside Crescent, Cuffley, Herts EN6 4QN

Introduction-The few previously reported studies of surfing injuries suggest that it is a relatively safe sport. We aimed to define the hospital workload created by such injuries in a region in which surfing is a popular local pastime and to survey those injured in an attemp to generate hypotheses as to why such injuries occur and how they may be prevented.

Method-All patients presenting with surfboard related injuries to hospitals in the Illawarra area of New South Wales between 1987 and 1993 were asked to complete a questionnaire regarding their injury.

Results-Data was collected from 403 patients (387 male, 16 female, mean age 19.7, age range 10-48 years). Patients were twice as likely to present on a weekend day compared with a weekday. Injuries presented throughout the year but peaked in January. Sixty one per cent of all injuries were caused by the surfe being struck by part of their own surfcrafttypically the fin. Stand-up, short boards were responsible for $76 \%$ of injuries. We found no evidence that any group of surfer was over-represented or under-represented on the basis of experience, competence, or hours spent surfing per week. Chronic injury from surfing was reported by $5.4 \%$ and chronic eye irritation by $7.2 \%$. Serious injury was uncommon. Only 16 patients required hospital admission and only 15 required formal operation. Most injuries were simple laceration requiring suture.

Conclusion-Although minor injuries are common we found little evidence of serious injury. Most injuries result from contact with the fins of the surfers own board. The use of a leg rope to attach the surfer to their board has increased recently. This can produce a degree of elastic recoil and may be implicated in the high prevalence of injuries caused by the surfer's own board.
Table 18 Performance of threshold heights for identifying major trauma

\begin{tabular}{lll}
\hline $\begin{array}{l}\text { Threshold height } \\
\geqslant \text { metres }\end{array}$ & Sensitivity & Specificity \\
\hline 10 & 0.17 & 0.97 \\
8 & 0.17 & 0.96 \\
6 & 0.25 & 0.94 \\
5 & 0.33 & 0.88 \\
4 & 0.42 & 0.75 \\
3 & 0.58 & 0.54 \\
2 & 0.83 & 0.24 \\
\hline
\end{tabular}

Can height of fall predict injury severity? S W Goodacre, M Than, E C Goyder, A Joseph

24 Brookside Crescent, Cuffley, Herts EN6 4QN

Introduction-Logic predicts that when a trauma victim falls from a height the likelihood of sustaining major injury will increase as the height increases. We aimed to examine the performance of height of fall as a predictor of major trauma in patients who have fallen from a height.

Method-A cohort of adult trauma victims attending the Royal North Shore Hospital emergency department between 1 January 1996 and 31 December 1997, having fallen from a height, was identified retrospectively by searching the hospital computer system. Height fallen, and injury severity score (ISS) were recorded. Major injury was defined as ISS $>15$. Thresholds were created using height fallen as a diagnostic cut off ranging from $2 \mathrm{~m}$ height to $10 \mathrm{~m}$ height. Sensitivities and specificities of each threshold to predict major trauma were calculated.

Results-166 cases were identified. The prevalence of major trauma was 0.07 . Table 18 shows the sensitivity and specificity of the thresholds used. At the optimal threshold of 5 $\mathrm{m}$ the positive predictive value is 0.17 . Using this threshold to predict major trauma will only identify one in three cases, while only one in six of those falling $5 \mathrm{~m}$ or more will have suffered major trauma.

Conclusion-Height of fall is a poor predictor of major injury. Even using a low threshold sensitivity is inadequate to rule out major injury while the low prevalence means that, even with impressive specificity at higher thresholds, the positive predictive value of the test is poor. Height fallen should not be used as a sole criterion for decision making.

Ulnar and median nerve neurapraxia in the hands of long distance tandem cyclists John Black, Emma Hormbrey, Philip Hormbrey

Accident and Emergency Department, fohn Radcliffe Hospital, Headly Way, Oxford OX3 9DU

Methods-A medical team of 19 cyclists who participated in a long distance charity tandem cycle ride from Land's End to John O'Groats and back were surveyed after the developmen of neurological symptoms in the hands of 10 members of the team.

Results-10 of 19 participants were male and spent a range of 1-30 days cycling and covered a distance of between 61 and 2032 miles during May 1997 on a pair of tandem bicycles. Ten members of the team had developed neurological symptoms in their hands by the end of the trip, which was bilateral in five. There were a total of 10 ulnar nerve neurapraxias in eight cyclists: four were predominantly sensory, one was motor, and five were mixed lesions. There were three unilateral median nerve neurapraxias, two had sensory symptoms only, and one was mixed. 
Nerve conduction studies in the first author demonstrated axonal degeneration in motor branch of the ulnar nerve consistent with compression/ischaemic injury in Guyon's canal. A repeat study six weeks after the injury confirmed significant improvement. The neurophysiological study in the third author demonstrated mild motor axon degeneration in the motor branch of the median nerve of the right hand, and mild axonal degeneration within the fasicles of the ulnar and median nerve of the left hand.

Conclusions - The risk of ulnar and median nerve neurapraxia in cyclists needs to be highlighted as cycling is becoming an increasingly popular recreational activity and long distance trips are becoming increasingly used as a vehicle for fund raising activities. Injuries to the dominant hand may have significant occupational consequences. Further research is needed to explore how modification in posture, glove and handle bar design may reduce the risks of these potentially very serious injuries from occurring.

It's a ring ting: manual $v$ motorised constricting band removal

Brad Peckler, Carl Hsu, Joanne Magro, Ka Sturmann

Beth Israel Medical Center, New York, USA

A constricting band on an appendage is a common emergency department presentation. Multiple reports describe a variety of methods for ring removal; none have studied manual versus motorised constricting band removal.

Objectives-This study evaluates the safety, efficacy, efficiency, as well as the preference of patient and provider of using a high revolution per minute (rpm) cutting device compared with a hand operated ring cutter for the removal of rings on appendages. We hypothesise that a high rpm device is the preferred method of ring removal.

Methods-This is a prospective, controlled, clinical observation trial. All patients who present with a constricting ring are enrolled in the study. The constricting band is opened at a single location using both the motorised (high rpm) device and the manual cutting device. If a second division is necessary the same procedure is followed. Data collected include the duration of each cut and preference of the patient and provider. Complications are likewise recorded.

Results-For this small pilot study $(\mathrm{n}=17)$, the mean (SD) time for the manual cutter was 304 (166) seconds and for the motorised cutter was 34 (35) seconds. Using a paired $t$ test, $\mathrm{p}<0.001$. The average time difference was 269 seconds $(95 \%$ confidence interval 176.7 to 362.1 ). Sixteen of the 17 of the patients and all providers preferred the motorised method. There were no serious complications.

Conclusions - Preliminary data suggest that the high rpm device is faster than the manual cutter and is preferable to both provider and patient. The complication rate is negligible.

Initial experience with the use of a chair to achieve rapid reduction of glenohumeral dislocation

J J M Black, G T Manivannan, P C Thomas, P Hormbrey

Accident and Emergency Department, fohn Radcliffe Hospital, Headly Way, Oxford OX3 9DU

Methods-One year retrospective observational pilot study to assess the success of reducing glenohumeral dislocation using a simple traction technique using a back of a chair in a single accident and emergency (A\&E) department without the use of intravenous analgesia or sedation.

Results - The method was attempted in 35 patients and was successful in $25(77.8 \%)$ patients. Ten patients subsequently received intravenous analgesia and sedation and reduction was achieved in eight patients using standard techniques. Two patients required open reduction. The mean duration of stay within the $A \& E$ department from arrival to discharge for those patients who were successfully reduced with the back of chair technique withou sedation was 82 minutes (SD 42, range 15-180 min) versus 197 minutes (SD 65.5, range 40-390 $\mathrm{min}$ ) in those requiring intravenous analgesia/sedation for conventional reduction methods $(p<0.001)$. There have been no clinical complications of the technique.

Conclusions - This technique is simple, rapid, and safe. It has been successful in over $75 \%$ of patients in our series. It significantly reduces the duration of stay within the A\&E department. We highly recommend this simple method of reduction as first line treatment for these injuries. We plan to perform a randomised prospective study to further define its role in the early management of shoulder dislocation and to design a chair to facilitate the procedure.

Monocyte surface L-selectin correlates with post-traumatic multiple organ failure T H Rainer, N Lam, R A Cocks

Accident and Emergency Medicine Academic Unit, Chinese University of Hong Kong and the Prince of Wales Hospital, Shatin, Hong Kong

Introduction-Leucocyte adhesion molecules are responsible for intercellular communication, mediate cell-cell and cell-matrix adhesion before migration and are upregulated early on circulating leucocytes after trauma. L-selectin mediates early leucocyte rolling over endothelium before migration. Posttraumatic complications are associated with leucocyte activition and early changes may reflect or predict subsequent insult. Thi study investigates whether alterations in the expression of leucocyte L-selectin correlates with multiple organ failure after injury.

Methods-Adult trauma patients admitted to the resuscitation room of the emergency department of a university hospital after a major mechanism of injury over an 18 month period were included. The presence and degree of multiple organ failure 48 hours after injury was determined using Goris' classification. Monocyte surface L-selectin was measured within three hours of injury by flow cytometry. Data were analysed using Spearman's rank correlations.

Results-This study included 120 patients with a mean age of 36 years (range 12-86 years, 97 males) and mean injury severity score of 17 (range 1-57). The organ failure score of 16 subjects was 23 . Significant correlations with multiple organ failure score were observed in both percent monocytes expressing $\mathrm{L}$-selectin $(r=0.309, \mathrm{Z}$ value $=2.8$ $\mathrm{p}<0.005)$ and monocyte mean fluorescen intensity $(r=0.363 ; Z$ value $=3.35 ; \mathrm{p}<0.001)$ There were also correlations in per cen lymphocytes expressing L-selectin $(r=0.263$ $Z$ value $=2.46 ; p<0.05)$ and average granulocyte expression $(r=0.216 ; Z$ value 2.00 $\mathrm{p}<0.05)$. No significant correlations with injury severity score or relationships with lung injury $(n=7)$ were identified.
Conclusions-Early increases in the expression of leucocyte L-selectin correlates with posttraumatic multiple organ failure and may be important in its subsequent development.

Severe trauma presenting to the resuscitation room of a Hong Kong emergency department

T H Rainer, S Y Chan, K Kwok, T K Suen, W Lam, R A Cocks

Accident and Emergency Medicine Academic Unit, Chinese University of Hong Kong and the Prince of Wales Hospital, Shatin, Hong Kong

Background-Little is known about the epidemiology or mortality after trauma in Hong Kong or of its demands on the service of emergency departments. Major trauma outcome studies have been initiated in the USA and UK but similar projects have not been reported from Hong Kong. This study investigates both the quantity and quality of trauma presenting to an emergency department in Hong Kong, informs on mortality and compares outcome to US standards.

Methods-A prospective observational study was conducted from January to June 1997 and included all trauma cases with the exception of thermal injury and drowning. There were 100000 new patient attendances at the emergency department of the Prince of Wales Hospital of which 227 trauma patients $(0.002 \%)$ were triaged to the resuscitation room. TRISS methodology was used to analyse data. The Z statistic was calculated to determine whether there was a statistically significant excess of unexpected deaths and the $M$ statistic calculated to determine whether this trauma population resembled the US Major Trauma Outcome Study baseline.

Results-Data was available on 221 (97\%) subjects. There were 203 adults (78\% male mean age 39 years, range 13-97) and 18 children ( $78 \%$ male, mean 8 years, range 1-12) Blunt injury accounted for $173(92 \%)$ cases and penetrating for $16(8 \%)$ cases. Moto vehicle accidents accounted for 96 (51\%) cases, falls for 47 (25\%) cases, assault for 18 (9\%) cases. Forty six (24\%) presented between the hours of midnight and $8 \mathrm{am}$. The median injury severity score (ISS) was 9 (mean 11, range 1-59) and median revised trauma score (RTS) was 7.8408 (mean 7.517 range $0-7.8408)$. Twenty three of $110(21 \%)$ subjects had an abnormal RTS (<7.8408). Overall mortality was $0 / 83(0 \%)$ after minor (ISS <9) trauma, $2 / 50(4 \%)$ after moderate (ISS 9-14) trauma, and 21/45 (47\%) after major (ISS >15) trauma. There were eight immediate deaths, seven early deaths $(<24$ hours), and eight late deaths ( $>24$ hours). TRISS analysis $(n=110)$ yielded a $Z$ statistic of $6.72(p<0.001)$. The $M$ statistic was 0.88 . Conclusion-Patterns of trauma in Hong Kong are typical of global reports. TRISS casemix resembles patterns observed in the USA. An excess in trauma deaths is unexplained and requires further investigation.

Why do patients get slow tracked to arthroscopy after an acute knee injury? John Ryan, Steve Barden, Sam Singh Accident and Emergency Department, Royal Sussex County Hospital, Eastern Road, Brighton $B N 25 B E$

Introduction - Patients with knee injuries commonly present to accident and emergency (A\&E) departments. After examination and investigation in the $A \& E$ department number of different options exist ranging from 
referral to orthopaedic surgeons for admission to discharge with no follow up.

Aims - The aims of this study were to evaluate a group of patients who underwent a knee arthroscopy at a time remote from their original injury and to produce recommendations based on these patients for when to seek specialist referral for patients who present with an acute knee injury.

Methods-The names of all patients who had an elective knee arthroscopy performed during a two year study period were cross checked with the A\&E department's computer database to identify which patients had previously attended with a knee injury. Information was collected from the case notes of all these patients.

Results-530 patients had knee arthroscopies performed of which 485 had elective arthroscopies. A total of 137 had previously attended the $A \& E$ department with injuries related to lower limb complaints; 77 had sustained knee injuries. Both $\mathrm{A} \& \mathrm{E}$ and hospital records were available for examination in 67 cases. Fracture clinic follow up was arranged for 44 patients. Eight were referred to their general practitioner, five for physiotherapy, and 10 were discharged. The mean time from presentation at the A\&E department to arthroscopy was four months. There was little correlation between findings at arthroscopy and the diagnosis, when stated, in the A\&E department.

Conclusions-The majority of patients who underwent an elective arthroscopy after presentation to the $A \& E$ department were managed appropriately by A\&E staff. A smaller number of patients with knee injuries had a delay in having an arthroscopy performed. Improved understanding of the mechanism of injury and interpretation of clinical signs may decrease the number of late referrals.

\section{Cervical immobilisation; are we achiev- ing it?}

Luke Houghton, Peter Driscoll

Emergency Medicine Directorate, Emergency Department, Hope Hospital, Stott Lane, Salford M6 $8 H D$

The use of semirigid cervical collars is considered essential for adequate cervical spine immobilisation. A study was carried out to investigate the immobilising effects of two cervical collars (Vertebrace and Stifneck Select) with and without head blocks and straps.

Twenty four healthy volunteers were used for the study and the measurements were taken in the four directions of neck movement: flexion, extension, lateral flexion, and rotation. All measurements were recorded using a hand held goniometer and a custom made device. Three results for each movement were obtained and the means calculated.

Overall, the collars produced a reduction in movement of between $31 \%$ and $45 \%$ which was significant at the $5 \%$ statistical level. The use of head blocks and straps produced a decrease in movement of between $58 \%$ and

Table 19

\begin{tabular}{|c|c|c|c|c|c|c|}
\hline \multirow[b]{2}{*}{ Dependent measure } & \multicolumn{3}{|c|}{ Baseline v exp } & \multicolumn{3}{|c|}{ Wooden v vacuum } \\
\hline & $F(2.76)$ & $p$ & $F(1.38)$ & $p$ & $F(1.38)$ & $p$ \\
\hline FVC & 12.21 & 0.001 & 34.65 & 0.001 & 0.00 & ns \\
\hline $\mathrm{FEV}_{1}$ & 30.51 & 0.001 & 41.96 & 0.001 & 5.66 & 0.022 \\
\hline \multicolumn{7}{|c|}{ Means and SD for dependent measure across conditions: baseline, wood, and vacuum } \\
\hline & Mean & $S D$ & Mean & $S D$ & Mean & $S D$ \\
\hline FVC & 2.72 & 0.98 & 2.34 & 0.91 & 2.33 & 1.04 \\
\hline $\mathrm{FEV}_{1}$ & 2.26 & 0.93 & 1.94 & 0.84 & 1.83 & 0.80 \\
\hline Comfort & $\mathrm{n} / \mathrm{a}$ & $\mathrm{n} / \mathrm{a}$ & 2.81 & 1.26 & 4.81 & 0.93 \\
\hline
\end{tabular}

$\mathrm{FVC}=$ forced vital capacity; $\mathrm{FEV}_{1}=$ forced expiatory volume in one second.

$64 \%$. Importantly, once head blocks and straps were used it appeared that the addition of a cervical collar was not beneficial. Furthermore, even when all the immobilising techniques were employed there remained a substantial amount of movement. This was most noticeable with extension where a reduction of only $20 \%$ to $30 \%$ occurred.

This study shows that head blocks and straps do not produce complete neck immobilisation. Furthermore a cervical collar does not contribute any additional benefit should the head blocks and straps be in place.

Application of clinical criteria for clearance of cervical spine injury by nurses Margaret Hsieh, Michael Gutman, Debbie Haliscak

Saint Francis Hospital and Medical Center, Hartford, CT, University of Connecticut Integrated Residency in Emergency Medicine, USA

Objectives-To determine whether registered nurses can clinically clear cervical spines as well as emergency physicians using a list of evidence based criteria, the following study was done.

Methods-Blunt trauma patients 12 years or older arriving with board and collar were prospectively enrolled in the study using a convenience sample. Registered nurses trained to clear cervical spine clinically and emergency physicians each completed a questionnaire for every patient with regard to the following criteria: (1) evidence of intoxication (2) altered consciousness, (3) posterior neck tenderness or pain, (4) distracting injury, (5) neurological deficit, and (6) need for cervical collar or cervical spine radiography. The $\kappa$ statistic was used to determine level of agreement between the two groups for each criterion and for needing collar and radiography.

Results - The $\kappa$ statistics for the six categories were $(n=128):(1) 0.89,(2) 0.88,(3) 0.72$, , (4) 0.45 , (5) 0.81 , and (6) 0.63 . There are 15 cases where the emergency physicians would clinically clear the patient but the registered nurses would not (registered nurse conservative) and eight cases in reverse. When disagreements where registered nurses were more conservative were changed into agreements, the $\kappa$ for criterion 6 becomes 0.88 .
Conclusion-This study showed good agreement between emergency physicians and registered nurses using criteria for clinically clearing cervical spines. Registered nurses were more conservative in clinically clearing the cervical spine; however, there were still unacceptable disagreements.

\section{Respiratory effects of spinal immobilisa-} tion

Vicken Y Totten ${ }^{\star}$, David B Sugarman ${ }^{\star \star}$ ${ }^{\star}$ Catholic Medical Center of Brooklyn and Queens, ${ }^{*}$ Rhode Island College, USA

Objectives-Does spinal immobilisation on a vacuum mattress inhibit respiration as much as a standard wooden backboard? Determine comfort differences.

Methods-Random order crossover study, 39 subjects. Baseline spirometry compared with wooden backboard and Philadelphia collar $v$ vacuum mattress and collar. Comfort on forced Likert scale. Data analysed by $3 \times 2$ mixed design analysis of variance, condition an intrasubject variable.

Results - The results of one way analysis of variance of three conditions (baseline, wooden board, and vacuum) are shown in table 19 .

Immobilisation restricted respiration about $15 \%$, similar under both conditions $(p=0.05)$. Forced expiratory volume in one second was less on the vacuum mattress. Respiratory restriction is a quadratic function, mor pronounced at age extremes. The vacuum mattress was more comfortable.

Conclusion-Our study confirmed that spinal immobilisation causes respiratory restriction. Vacuum mattresses were more comfortable than wooden back boards.

Successful non-operative treatment of adults with blunt splenic injuries

V Wignakumar, E Cumberland, W E Charash, M Prince, J B Ochoa, P A Kearney Section of Trauma and Critical Care, Department of Surgery, Room C-223, University of Kentucky, Chandler Medical Center, 800 Rose Street, Lexington, KY 40536-0084, USA

Objective-To evaluate the current approach and the efficacy of non-operative management of blunt splenic injuries.

Table 20

Operative: 62 patients (one third)

Non-operative: 102 patients (two thirds)

Group A: Splenectomy, 34

Group B: Splenorrhaphy, 20

Group C: Laparotomy ${ }^{\star} 8$

* for other indications (incidental findings of splenic injuries required no treatment)

\begin{tabular}{llllll}
\hline Group & $A$ & $B$ & $C$ & $D$ & $E$ \\
\hline Mean ISS & 28 & 22 & 24 & 18 & 21 \\
Mean AIS (AB) & 3.5 & 2.6 & 2.6 & 2.4 & 3.4
\end{tabular}


Method-Three year retrospective analysis of blunt splenic injuries at a level I trauma centre. On the basis of our ongoing practice, after initial fluid management, all haemodynamically unstable patients and those requiring another emergent operation (for example, neuro, cardiothoracic) underwent DPL and were treated accordingly. All haemodynamically stable patients with suspected abdominal injuries underwent computed tomography. These patients were treated non-operatively, regardless of the degree of splenic injury, age, or Glasgow coma scale score. Most of these patients were admitted to the intensive care unit and were closely monitored. Non-operative management was considered to have failed if patients had evidence of continuous haemorrhage or required laparotomy for associated abdominal injuries.

Results-During the three year period, 189 patients with blunt splenic injuries were evaluated. Of these patients, 25 died (18 immediate deaths in the emergency room or operating room and seven late deaths, none of which were attributed solely to splenic injuries). Table 20 shows the treatment the remaining 164 patients received.

Conclusion-On the basis of our experience, we conclude that approximately $84 \%$ of blunt splenic injuries can safely be managed in the non-operative group. Initial haemodynamic stability is an important predictor for successful non-operative management. The spleen can be salvaged in more than $75 \%$ of trauma cases.

\section{Posters: Pre-hospital Care}

How long does it take to do practical procedures out-of-hospital?

H R Guly, B Barrett

Accident and Emergency Department, Derriford Hospital, Plymouth PL6 8DH

Introduction-Ambulance paramedics no longer "scoop and run" but are equipped to assess and attempt to stabilise a patient pre-hospital. However there is a cost to this The patient may be delayed reaching hospital where they can obtain definitive care (particularly in trauma) and there are operational costs in that an ambulance delayed on scene is unavailable for the next emergency. When drawing up protocols, it is important to know the time implications of recommended procedures.

Method-This was an observational study in which a research assistant travelled on front line ambulances and (among other things) timed procedures that were carried out.

Results-There is much variation but the median times for procedures are shown in table 21 .

How accurate are the on scene times obtained from the ambulance computer? H R Guly, B Barrett

Accident and Emergency Department, Derriford Hospital, Plymouth PL6 8DH

Introduction-Ambulance on scene times (OSTs; and response times) are important. If they are to be used for research or monitoring standards, their accuracy needs to be confirmed.

Method-As part of a prospective study on ambulance OSTs, a research assistant travelled with emergency ambulances investigating different aspects of OSTs including data on their accuracy.
Results-The OST as recorded by the computer and the actual OST were compared in 265 emergency and 61 urgent calls. In only $36.2 \%$ did the OST as recorded by the computer match the real OST exactly. In $1.5 \%$ the computer registered a shorter OST and in the remaining $62.2 \%$ the computer OST was longer than the actual OST. The median percentage difference between the computer and the actual OST was $1.7 \%$. However there was a wide distribution and in three cases the computer OST was more than $100 \%$ higher than the actual OST.

Discussion-The differences between the computer and the actual OST are generally small but a few cases show a large difference between the times. We conclude that when studying large numbers of incidents, it is safe to use the computer OST as an indicator of actual OST as long as it is accepted that there will be a small inaccuracy. It would not be safe to use the computer OST when studying small numbers of incidents as there is a risk that one or two significant errors would distort the results. In almost every case the longer recorded OST was the result of ambulance control being notified that the ambulance was on scene before it actually arrived. Thus these errors in the computer OST will be matched by the computer showing response times shorter than actually occur.

Patient's use of pain relief before arrival at the accident and emergency department

S Derbyshire, A J Gray

16 St Wilfred's Drive, Healey, Rochdale OL12 $6 D B$

Background-Patients who self refer and are considered as "minor injuries" form the bulk of attendances in most UK accident and emergency (A\&E) departments. Pain is nearly always a symptom in these patients. Pain relief is one of the prime objectives of healthcare and, in this group, self medication before arrival is important in achieving this.

Objective-We aimed to determine what proportion of patients had taken analgesia before their arrival with a "minor injury" at our A\&E department.

Method -A random sample of adult patients who self referred as "minor injuries" was carried out. At triage, patients were offered analgesia where appropriate, and then asked to participate if they complained of pain or had suffered an injury. Treatment of individuals was not influenced and replies were anonymous. Patients were asked whether they had taken pain relief before attending the $A \& E$ department. If they had not, they were asked

to give a reason from a list why they had not. They were asked how long they had been in pain before presentation.

Results-292 survey forms were completed; 235 patients $(80 \%)$ had not taken analgesia before attendance. In this group, the reasons were as follows: the pain was not severe enough, $50 \%$; the patient did not have time to take pain relief, $17 \%$; no pain relief was available, $16 \%$; the patient believed the pain relief would harm them, $7 \%$; the patient did not believe in taking pain relief, $6 \%$; the patient thought that the doctor would not believe that they were in pain, $3 \%$. Fifty eight per cent of respondents had been in pain less than 12 hours before attending.

Conclusions-20\% of patients with painful conditions or injuries take analgesia before attendance at our A\&E department; $50 \%$ did not because they did not consider the pain severe enough. Most patients had been in pain for less than 12 hours before presentation. Patient education is needed to encourage the use of pain relief before attendance and the effectiveness of such a health promotion programme should be assessed.

Use of a geographic information system to determine the most appropriate means of transport to a regional trauma centre E Brooke Lerner, Anthony J Billittier IV, Jeremy Sikora, Ronald M Moscati

Department of Emergency Medicine, School of Medicine and Biomedical Science, State University of New York at Buffalo, NY, USA

Objective-To determine if a geographic information system (GIS) and historical data can be used to create a map that identifies locations (zones) from which ambulance or helicopter transport will result in shorter prehospital times.

Methods-A retrospective, cross sectional review of a trauma registry was conducted to gather data for all patients transported directly to the trauma centre between 1993 and 1996 Incident locations and times from first 911 contact until arrival at the trauma centre (prehospital time) were extracted. A GIS was used to create a reference map with all inciden locations plotted and given a $\mathrm{z}$ coordinate for time. Two contour surfaces were interpolated: one for all helicopter transports and one for all ground transports. Areas where the helicopter surface was lower than the ambulance surface were designated air zones since helicopter transport resulted in shorter pre-hospital times. The remaining areas were designated ground zones since ambulance transport resulted in shorter pre-hospital times. Average pre-hospital times were calculated for each

Table 21

\begin{tabular}{lll}
\hline & No & Median time (min) \\
\hline Assessments & 256 & 1.2 \\
History taking & 148 & 0.7 \\
Basic observations & 72 & 2.0 \\
Blood pressure measurement & 61 & 2.0 \\
ECG monitoring & 76 & 0.6 \\
Pulse oximetry & 13 & 3.1 \\
Blood sugar measurement & & \\
Simple treatments & 54 & 1.0 \\
Basic airway care & 15 & 1.4 \\
Cervical collar & 18 & 2.3 \\
Dressings & 13 & 1.1 \\
Entonox & 9 & 1.8 \\
Splint upper limb & 8 & 4.4 \\
Splint lower limb & & \\
Advanced treatments (all attempts) & 12 & 1.8 \\
Endotracheal intubation & 43 & 3.6 \\
Intravenous cannulation & 19 & 1.8 \\
Drugs (excluding cardiac arrest) & &
\end{tabular}


mode of transport in both zones and were compared using a two tailed $t$ test.

Results-Multiple air zones were identified beginning between five and 16 miles from the trauma centre and were dissected by expressways. Mean pre-hospital time from the helicopter area was $50 \pm 9$ minutes for helicopter transport $(n=54)$ and $63 \pm 14$ minutes for ambulance transport $(n=140)$. The mean difference was 13 minutes $(p<0.000001 ; 95 \%$ confidence interval (CI) 8.95 to 17.05). Mean pre-hospital time from the ground zone was $68 \pm 16$ minutes for helicopter transport $(n=122)$ and $32 \pm 14$ minutes for ambulance transport $(n=2047)$. The mean difference was 36 minutes ( $p<0.000001 ; 95 \%$ CI 33.59 to 38.41).

Conclusions-A GIS and historical data can create a map identifying locations from which helicopter or ambulance transport will minimise pre-hospital time. Inappropriate choice of transport mode significantly increased prehospital time.

Pre-hospital personnel agree poorly with emergency physician on clinically excluding cervical spine injury

Margaret Hsieh, Michael Gutman, Debbie Haliscak

Saint Francis Hospital and Medical Center, Hartford, CT, University of Connecticut Integrated Residency in Emergency Medicine, USA

Objectives-This study was done to determine whether EMTs reliably agree with emergency physicians on clinically clearing cervical spines. Methods-Blunt trauma patients 12 years or older arriving with board and collar were prospectively enrolled using a convenience sample. EMTs trained to clear cervical spines clinically and emergency physicians each completed a questionnaire for every patient with regard to the following criteria: (1) evidence of intoxication, (2) altered consciousness, (3) posterior neck tenderness or pain, (4) distracting injury, (5) neurological deficit, and (6) need for cervical collar or cervical spine radiography. The $k$ statistic was utilised to determine level of agreement between the two groups for each criterion

Results-The $\kappa$ statistics for the six categories were $(\mathrm{n}=36)$ : (1) 0.83 , (2) 0.9 , (3) 0.56 , (4) 0.17 , (5) 0.94 , and (6) 0 . For category 6 , EMT-Ps have higher level of agreement with emergency physicians $(0.23, \mathrm{n}=13)$ than EMT-Is $(-0.25, \mathrm{n}=8)$ and EMT-As $(-0.33$, $\mathrm{n}=12$ ). If acceptable disagreements (EMT not clearing and $\mathrm{MD}$ clearing) were considered as agreements, $\kappa$ for criterion 6 became 0.85 (excellent), 0.25 (poor), and 0 (poor) for EMT-P, EMT-I, EMT-A respectively. EMT-As and Is would unacceptably clear $45 \%$ of patients and EMT-Ps $8 \%$.

Conclusion-The agreement between emergency physicians and EMTs in using a set of criteria for clear cervical spine clinically is dangerously poor. The level of agreement improves with the level of EMT training.

\section{A survey of emergency medical service} palliative care protocols in the US

Steve Ausband, Juan A March, Lawrence H Brown

East Carolina University School of Medicine, Department of Emergency Medicine, Division of EMS, USA

Objective-Do not resuscitate protocols are common in out-of-hospital care, yet little has been published regarding palliative care protocols in the emergency medical service (EMS) environment. The purpose of this study was to gather information on the number of EMSs that provide a specific protocols for palliative care patients in the out-of-hospital setting.

Methods-A letter asking for any palliative care protocols utilised by EMS, was mailed to 249 EMS agencies providing care to the 200 largest cities in the US. Telephone follow up was made with those agencies not responding to the initial mailing. All palliative care protocols were examined for similarities and differences. In addition, the total population served by those agencies with a palliative care protocol was also calculated.

Results-A total of 159 responses were obtained, through the combined initial mailing and telephone follow up, for a response rate of $159 / 249(64 \%)$. Only 42 of the $159(15.6 \%)$ responding agencies had a palliative care protocol. The population of the US is estimated at 265 million. The population of the cities surveyed was estimated at 57 million, or $21 \%$ of the total US population. Those 200 largest cities with a palliative care protocol represent 9.7 million, or $17 \%$ of the US population surveyed by the study.

Conclusion-Unlike do not resuscitate protocols, palliative care protocols are not common in the US. The need for out-of-hospital palliative care protocols should be examined further with in the EMS community similar to that which is already in place for do not resuscitate.

Emergency medical service and emergency department interactions: problematic areas identified

Robert F Carroll, Michael Drescher, Sara Knuth, Raffaella Coler

University of Connecticut School of Medicine, Hartford Hospital,USA

Objective-Little has been written about the interaction between the pre-hospital emergency medical service (EMS) and the emergency department from the perspective of EMS providers. We undertook a study to identify which aspects of the EMS and emergency department interaction are problematic and potentially correctable.

Methods-Over a seven month period, we conducted a survey of approximately 600 EMS providers certified as either EMT, EMT-I, or EMT-P. To date 112 responses were received from providers who routinely transport patients to the emergency department. We received responses from $45 \mathrm{EMTs}$, 21 EMT-Is, and 41 EMT-Ps. The setting was an urban teaching hospital emergency department with an annual census of 62000 . The survey addressed three major areas: radio and communications, interactions with emergency department staff, and equipment and supplies. The respondents quantified their satisfaction in these areas using a five point scale ranging from very poor $=1$ to very good $=5$. Results - Our survey identified six points of dissatisfaction in the EMS-emergency department interface. (1) Availability of emergency department physician for medical control (mean satisfaction (MS) $2.88 \pm 1.86, n=41$ ). (2) Physician willingness to approve orders for medication administration (MS 2.18 $\pm 2.02, n=41$ ). (3) Physician awareness and understanding of EMS controls (MS 2.58 $\pm 1.89, \mathrm{n}=41$ ). (4) Attitude and attentiveness of the triage nurse upon arrival (MS 2.91 $\pm 1.15, \mathrm{n}=112$ ). (5) Availability of patient care nurse for report (MS $2.67 \pm 1.11, n=112$ ). (6) The security of
EMS equipment left in the emergency department (MS 2.98 $\pm 1.29, \mathrm{n}=112$ ).

Conclusion-Several areas of the EMSemergency department interaction have potential for improvement. We believe that increasing EMS provider satisfaction in the emergency department will translate into higher job satisfaction and ultimately benefit patient care. Further research is needed to determine which interventions will be most effective to this end.

What is the layperson definition of an emergency?

Robert W Derlet, Alicia M Ledesma

University of California Davis, USA

Objective-To determine the public's perception as to the general definition of emergency medical condition (EMC).

Methods-A survey of a random sample of persons at shopping malls and on the street was conducted over a six month period in 1997 in California. Individuals over age 18 were asked in person to complete a survey sheet. This form asks persons to choose one of four definitions of "emergency medical condition." These include (1) the 1986 federal definition: a condition which may result in death, permanent disability, or causes severe pain; (2) federal definition and any condition that prevents work; (3) federal definition and any condition needing attention outside normal business hours; and (4) any condition at anytime as determined by the patient. Demographic information was also collected. Health care providers were excluded.

Results - A total of 1018 persons completed survey sheets in several locations in Northern California. EMC was defined as follows: (1) strict federal definition, $48.7 \%$; (2) federal definition and conditions preventing work, $3.0 \%$; (3) federal definition and any conditions outside business hours, $16.5 \%$; and (4) any condition at anytime as determined by the patient, $31.6 \%$. Combining choices 3 and 4 reveals $48.1 \%$ of the public who feel that during off hours any medical problem needs emergency department care which is not statistically different from option 1 alone. Options 1, 2, 3, and 4 were all statistically different when comparisons were made $(p \leqslant 0.01)$. No significant difference in answers occurred when age groups or occupations were compared.

Conclusion-The public has split views concerning the definition of EMC. Approximately half use the conservative federal definition, and half use patient self determined need as the definition.

Pre-hospital invasive haemodynamic monitoring to guide cardiopulmonary resuscitation: preliminary experience of a physician response system

James E Manning, Greg D Mears, Marshall McCoy, D Neil Batson, Thomas R Griggs

Department of Emergency Medicine, School of Medicine, University of North Carolina at Chapel Hill, NC, USA

Objective-Cardiopulmonary resuscitation (CPR) diastolic coronary perfusion pressure (CPP) is the most accurate indicator of myocardial perfusion during CPR. This study reviews early clinical experience with physician dispatch to out-of-hospital cardiac arrests (OOHCA) and pre-hospital invasive haemodynamic monitoring (PHIHM) to adjust CPR and ACLS. 
Methods-The setting was a suburban/rural county with a principal city having a population of 60000 . We retrospectively reviewed medical records of OOHCA victims having thoracic aortic and central venous catheterisation attempted after initial CPR and ACLS had failed. Force, rate, and duty cycle of CPR were adjusted in order to maximise CPP. Aortic epinephrine (AoE) was given if intravenous or endotracheal epinephrine did not result in return of spontaneous circulation (ROSC). Parameters were physician response time (MD-RT), initial CPP, maximal CPP, any ROSC, hospital admission, and survival to discharge.

Results-There were 13 physician dispatches. Six patients had been in prolonged unwitnessed arrests or had living wills declining CPR. Seven witnessed OOHCA victims underwent PHIHM. MD-RT was $12 \pm 5 \mathrm{~min}$. Initial CPP was $10 \pm 7 \mathrm{~mm} \mathrm{Hg}$ and with adjusted CPR/ACLS maximal CPP was $29 \pm 9$ $\mathrm{mm} \mathrm{Hg}(\mathrm{p}<0.05$, analysis of variance). Overall, five of seven had at least brief ROSC, three of seven survived to hospital admission, but none of seven survived to discharge. For MD-RT $<12 \mathrm{~min}$, three of four survived to hospital admission, whereas for MD-RT $>12$ $\mathrm{min}$, none of three survived to hospital admission. Prompt ROSC after AoE was seen in two patients with hospital admission. The force and rate of CPR resulting in maximal CPP varied on an individual basis.

Conclusion-PHIHM allowed for individualized adjustment of CPR and ACLS which increased CPP. Rates of ROSC and hospital admission were greater than historical experience. However, a beneficial effect of PHIHM on discharge has not yet been demonstrated. Response to AoE appeared favourable. Early intervention appears to be crucial.

\section{Implementation of a pre-hospital 12 lead} program: does it reduce the time to reperfusion using thrombolytic therapy? Sara F Sutherland, Robert O'Connor, Scott P Krall

Department of Emergency Medicine, Union Hospital, Elkton, Maryland, Department of Emergency Medicine, Medical Center of Delaware, Newark, Delaware, USA

Objectives-Out-of-hospital electrocardiograms (ECGs) are used to diagnose acute myocardial infarction (AMI) before hospital arrival. We conducted this study to the effect that out-of-hospital 12 lead ECGs and eligibility screening for thrombolytic therapy (TT) had on emergency department treatment times.

Methods-This study is a prospective analysis of all patients receiving TT for AMI in a community hospital emergency department setting. Study period was from 1 July to 31 December 1997. Patients treated by paramedics with 12 lead capability constituted the study group, whereas those arriving by private vehicle or by paramedics without 12 lead capability constituted the control group. All ECGs were interpreted by paramedics and arrived at the emergency department with the patient. Each group of patients had the

Table 22

\begin{tabular}{lllll}
\hline & Door:ECG & ECG:decision & Decision:TT & Total \\
\hline ECG group $(\mathrm{n}=13)$ & 8.5 & 10.5 & 9.3 & 28.3 \\
Control $(\mathrm{n}=13)$ & 7.3 & 13.7 & 22.7 & 43.7 \\
p Value & $\mathrm{NS}$ & $\mathrm{NS}$ & 0.004 & 0.01 \\
\hline
\end{tabular}

Values are in $\min . \mathrm{NS}=$ not significant.
Table 23

\begin{tabular}{lll} 
Success/attempt & $N T I(\% \pm C I)$ & $O T I^{\star}(\% \pm C I)$ \\
\hline Control & $36 / 54(67 \pm 13)$ & $784 / 856(92 \pm 2)$ \\
Investigational & $19 / 21(90 \pm 13)$ & $378 / 431(88 \pm 3)$
\end{tabular}

^Both groups used conventional ETTs for OTI.

following time intervals determined: door to (emergency department) ECG, ECG to decision (to give TT), decision to drug, door to drug, and ECG to drug. All out-of-hospital ECGs were confirmed on emergency department arrival. Data were analysed using MannWhitney.

Results-During the study period, 26 patients were given TT for AMI in the emergency department. The time intervals (in $\mathrm{min}$ ) are shown in table 22 ("total" = door to drug interval).

Conclusions-There is a significant decrease in control group. Most of the time savings is attributable to a reduction in the time spent between decision and administration of TT. Out-of-hospital ECGs can reduce door to drug time by permitting the staff to prepare in advance for patient arrival, thereby streamlining emergency department care. intubation is improved with the use of endotracheal tube directional tip control Robert E O'Connor, Marianne Bitner, Robert Ross, Ross E Megargel, Dean Dobbert, Elsburgh O Clarke, John F Madden, Dennis Jacobs, Lloyd Massey

Department of Emergency Medicine, Medical Center of Delaware, Newark, Delaware, Delaware EMS Office, Department of Public Health, Dover, DE, USA

Objectives-Nasotracheal intubation (NTI) is used to secure the airway in patients who are spontaneously breathing. The success rate for NTI is often lower than for orotracheal intubation (OTI). We conducted this study to determine whether the use of an endotracheal tube (ETT) capable of directional tip control improves the NTI success rate.

Methods-This prospective, experimental study was conducted by a state emergency medical service agency during 1996 and 1997. Consecutive patients undergoing attempted NTI or OTI were included. Five paramedic agencies were trained to use an ETT with trigger activated distal tip directional control for NTIs (investigational group). Ten agencies used conventional ETTs for NTIs and served as concurrent controls (control group). Both groups used conventional ETTs for OTIs. An intubation attempt was defined by tube passage, and success was defined as confirmed endotracheal placement. Statistical analysis was performed using $\chi^{2}$ and determining the $95 \%$ confidence interval (CI).

Results-A total of 1362 intubation attempt were studied. The results are shown in table 23.

Overall success rate was $89 \%$.

Conclusions - Conventional ETTs have a lower success rate for NTI than for OTI. Using an the door to drug time between the study and

Paramedic success rate for nasotracheal
ETT with distal directional control significantly improves the success rates for NTIs to a level comparable with OTI using conventional ETTs. Whether ETTs with distal directional control can be used to improve the success rate for OTI remains to be determined.

Paramedic $v$ emergency physician decisions regarding the need for emergency department evaluation

Scott M Sasser, Melissa Brokaw, Thomas H Blackwell

Carolinas Medical Center, Charlotte, North Carolina, USA

Introduction-Emergency medical service systems have failed to escape reform efforts aimed at providing cost effective healthcare. One potential area of cost containment focuses on the issue of ambulance misuse; prior studies have reported the incidence of inappropriate transport to be $40-50 \%$. The concept of paramedic initiated refusal of transport has been the subject of limited research, and raises many ethical, social, and legal dilemmas.

Purpose-To compare paramedic and emergency physician decisions regarding the need for emergency department evaluation.

Methods-Observational, non-interventional survey of paramedics and emergency physicians conducted in an urban, single tiered, all ALS system. Paramedics completed a survey after patient transport. Emergency physicians completed the same survey after initial emergency department evaluation. All patients transported to our facility were eligible for enrollment. The groups were blinded. All patients were transported. SAS v6.12 was used for analysis.

Results-3347 patients were eligible; 509 $(15 \%)$ had surveys completed by both groups. These were analysed for agreement, which was defined as the same answer to the question: "Did this patient need to be seen in the emergency department?". Observed agreement was $0.68(345 / 509), 95 \%$ confidence interval $(\mathrm{CI}) \pm 4 \%, \kappa=0.303,95 \% \mathrm{CI}$ \pm 0.077 . Of the 509 , paramedics indicated evaluation was necessary in $318(62 \%)$, not in $174(34 \%)$, and that they were unsure in 17 (3\%). Emergency physicians indicated evaluation was necessary in $373(73 \%)$, not in 117 $(23 \%)$, and that they were unsure in $19(4 \%)$. When paramedics indicated that emergency department evaluation was necessary (318), emergency physicians agreed in 271 (85\%) cases. When paramedics indicated that emergency department evaluation was not warranted (174), emergency physicians disagreed in $91(52 \%)$ cases.

Conclusions-Disagreement existed between paramedics and emergency physicians regarding the need for emergency department evaluation. Of particular concern were the 91 cases where paramedics may have refused transport, and emergency physicians disagreed. Further research may reveal select, low risk patients who may be refused transport.

Comparison of two methods of data collection for key out-of-hospital data elements: poor agreement

Steven S Andrews, Kevin Chu, Robert A Swor, Raymond E Jackson, Rebecca G Pascual

William Beaumont Hospital, Wayne State University School of Medicine, USA

Out-of-hospital cardiac arrest (OOHCA) data element are well defined. The methodology 
for obtaining those data elements has not been well studied.

Objective-We sought to determine the interrater reliability between emergency medical service (EMS) run sheet abstraction and telephone interviews of the person who identified the cardiac arrest. We chose the elements of witnessed arrest and bystander cardiopulmonary resuscitation (CPR), as these are major predictors of outcome.

Methods-A prospective, observational study of OOHCA cases brought to two suburban hospitals from $9 / 96$ to $11 / 97$. Data from OOHCA cases were retrieved by two methods. A single abstractor retrospectively retrieved data from EMS run sheets. The person who first discovered the arrest victims were identified on the day of arrest and interviewed by phone two weeks after the event by one of the authors (RP). The level of agreement was estimated using Cohen's $\kappa$ statistic.

Results-During the study period, 85 cases had both an abstracted runsheet and a phone interview. The level of agreement concerning witnessed arrest was very poor, $k=0.19$. The agreement on bystander CPR was poor, $\kappa=0.43$.

Conclusion-For this sample of OOHCA, the different methods of data collection had poor agreement. Data retrieval methodology may contribute to the substantial variation seen in OOHCA research.

\section{Posters: Toxicology}

Chemical incidents. An accident and emergency perspective

Simon J Ward, Virginia Murray

Chemical Incident Response Service, Medical

Toxicology Unit, Guy's and St Thomas' Hospital

Trust, Avonley Road, London SE14 5ER

The Chemical Incident Response Service (CIRS), based at the Medical Toxicology Unit, Guy's Hospital, provides a multidisciplinary 24 hour emergency service for advice on the management of chemical incidents. There were 931 incidents reported to CIRS during 1997 , and over $60 \%$ of these were reported by hospitals. Accident and emergency (A\&E) departments, being in the front line, bear the brunt of these incidents. Although the number of incident reports are rising year on year, each individual $A \& E$ department may have limited experience of dealing with an incident in its own locality.

There have been five recent chemical incidents in the last six months, for which CIRS has been requested for advice, where the $A \& E$ department and its staff have been significantly contaminated and the inciden has resulted in closure of the $A \& E$ department for variable amounts of time. These incidents have lead to considerable disruption of services and presented significant hazards to $A \& E$ staff and patients already in the department. A number of potentially avoidable problems have been identified by CIRS. These include secondary contamination and consequent illness of $A \& E$ staff, and dissemination of chemical contamination into other parts of the hospital outside the A\&E department. Follow up of these incidents and feedback from debriefing sessions, as well as CIRS's increasing experience of advising on the management of incidents outside hospitals, has led CIRS to develop guidelines on the management of chemical incidents that present to the $A \& E$ department. These may help to enhance the awareness of safety issues and the optimal management of a chemical incident, while also disseminating best practice, so that the hazards to patients and staff can be minimised.

\section{$\boldsymbol{N}$-acetylcysteine use in poisonings other} than paracetamol. A review

Simon J Ward

National Poisons Information Service (London), Medical Toxicology Unit, Guy's and St Thomas' Hospital Trust, London SE14 5ER

Objective-The National Poisons Information Service (NPIS) in London received over 200000 calls during 1997, of which over $10 \%$ involved paracetamol (acetaminophen) either alone or in combination with other potential poisons. For many of these calls NPIS (London) advised the use of $\mathrm{N}$-acetylcysteine (NAC), however, there are many published reports for the use of this antidote for less agreed or widely known causes of poisoning. The value of these other toxicological uses for NAC are established to varying degrees with some recommendations being based on anecdote and others on significant theoretical and/or clinical evidence. The aim of this review is to evaluate and clarify this evidence for these alternative uses of NAC.

Methods-The literature and data available at NPIS (London) were searched. Compounds for which NAC has been recommended, but not necessarily by NPIS (London), include (in alphabetical order): acrylonitrile, alkalis, amanitin poisoning, arsenic, bromobenzene, carbon tetrachloride, chloroform, clove oil, 1-2,dichloropropane, dichromate and chromium poisoning, gold, mercury, methyl bromide, naphthalene, phosgene, potassium permanganate, sulphur mustard, trichloroethylene, and tetrachloroethylene.

Results - The evidence is divided into levels of rigour. Group 1 contains compounds for which NAC is definitely recommended by NPIS (London) because there is sound theoretical evidence and clinical experience for usage. Group 2 contains compounds for which there is good theoretical evidence for effectiveness but with limited clinical experience. Group 3 contains compounds for which there is good theoretical evidence for the effectiveness of NAC, but only experience from animal studies. Group 4 contains compounds for which there is limited theoretical evidence and anecdotal human experience. Group 5 contains compounds for which there is limited theoretical evidence and only animal experience. Group 6 contains compounds for which there is minimal theoretica evidence or clinical experience. Group 7 contains compounds for which there is evidence of efficacy, but there are other more efficacious agents.

Conclusions-Based on a review of the evidence available, there are significant and sound uses for NAC, while other uses can only be regarded as experimental or anecdotal. In addition, some uses for NAC have been superseded by more efficacious agents. Thi paper also provides a structured typology which allows the available evidence to be classified into groups of similar levels of rigour. This typology may be used for evaluating the use of other antidotes, especially as randomised control trials of poisoned patients are often not ethically possible.
A review of inquiries to the National Poisons Information Service (London centre) regarding paediatric ingestion of fragrance oils

\section{J M Butler, J N Edwards}

National Poisons Information Service (London), Guy's and St Thomas' Hospital Trust, Medical Toxicology Unit, Avonley Road, SE14 5ER

Essential oils are volatile odorous complex mixture of esters, alcohols, aldehydes, ketones, and terpenes derived from plants. Historically they have been used as perfumes and flavourings, and in treatment of various medical conditions. Medicinal use is now limited but their inclusion in a variety of aromatherapy products, fragranced pot-pourri arrangements, and burner oils means they have a wide availability and popularity. In the above products the oils are diluted with nonvolatile carrier oils. As essential oils have the potential to cause serious effects on ingestion so there is a potential risk from such products.

In 1996, the London centre of the National Poisons Information Service (NPIS) received 602 calls relating to ingestion of fragrance/ aromatherapy oils. As no case was classified as serious, it was felt that the toxicity of these products had been overestimated. Over the subsequent 16 months a postal survey was performed to determine the potential toxicity of these oils. For any childhood ingestion of fragrance oils presented to the accident and emergency department, the caller was sent a questionnaire to determine severity, management, and outcome of the case.

The survey achieved a return rate of $49.5 \%$. Of these cases $64 \%$ remained asymptomatic. The most common effects reported were buccal irritation and gastrointestinal disturbances. Drowsiness was reported in two cases but no further symptoms were observed. Of the symptomatic cases reported, effects were attributed, by the treating physician, to pre-existing illness.

These results suggest a relatively low toxicity. However, in no case were ingestions confirmed analytically, and further investigation of these products may be merited.

Chemical incident response by the Medical Toxicology Unit

R MacLehose, $H$ Wheeler, V Murray

Chemical Incident Response Service, Medical Toxicology Unit, Guy's and St Thomas' Hospital Trust, Avonley Road, London SE14 5ER

Objectives - The Chemical Incident Response Service (CIRS), established in 1996, is part of the Medical Toxicology Unit which also includes the National Poisons Information Service (NPIS) (London). The service holds contracts with 74 health authorities throughout six regions in England, and provides assistance in the event of a chemical incident. In 1997 the service responded to over 930 chemical incidents. The aim of this paper is to advise emergency personnel of the services and information available and to highlight the part that emergency staff play in the event of a chemical incident

Methods-Data from the CIRS database of chemical incidents in 1997 was reviewed and evaluated by "inquirer type" and types of information requested.

Results-CIRS provides 24 hour toxicological information and advice on medical management of the contaminated patient. Over $60 \%$ of the incidents in 1997 were identified through inquiries from accident and emergency (A\&E) departments; these incidents 
were cascaded to CIRS through the NPIS (London). The remaining $40 \%$ of inquiries were generated by public health departments and other health professions such as general practitioners, dentists, and environmental health officers.

Conclusions - CIRS current functions are:

- Advise on assessment of the nature of the chemical hazard;

- Advise on health effects from exposure to the hazard;

- Decontamination and treatment of patients with adverse health effects due to chemical exposure;

- Advise on collection and analysis of biological and environmental samples as well as suspect material

- Follow up of individual cases, with medical toxicological assessments;

- Undertaking of literature surveys and reviewing sources of information;

- Provision of training materials for A\&E, public health, and environmental health departments;

- Specialist training days for A\&E staff on issues such as decontamination, emergency planning, and chemical triage.

Current experience from CIRS has shown the need for closer collaboration, cooperation, and communication between CIRS, A\&E departments, public health and the blue light services in the response to chemical incidents.

\section{Crowd control agents emergency case} inquiries

H Wheeler, R MacLehose, E Euripidou, V Murray

Chemical Incident Response Service, Medical Toxicology Unit, Guy's and St Thomas' Hospital Trust, Avonley Road, London SE14 5ER

Objectives-The National Poisons Information Service (NPIS) (London) and the Chemical Incident Response Service (CIRS) received over 600 inquiries relating to crowd control agents such as CS gas in the first eight months of 1997; this is a twofold increase since 1994. This increase may be due to the fact that in March 1996 CS incapacitant sprays were issued to 16 police forces on a trial basis. These trials have been completed and the majority of police force throughout England are carrying CS incapacitant sprays.

Crowd control agents are designed to have an immediate effect; they are an irritant to the skin, eyes, and the respiratory tract. These effects are meant to be short lived and self limiting. The aim of this study is to identify the proportion of total calls relating to crowd control agents and the proportion of which were used by police.

Methods-A retrospective review of all inquiries made to the NPIS (London) in 1997 wa undertaken. Calls relating to crowd control agents were selected and collated. The data were analysed for caller type, agent implicated, and patient details.

Results $-95 \%$ of all the inquiries relating to crowd control agents are generated by accident and emergency (A\&E) departments. Of all these inquiries between January and August 1997, police incapacitant sprays were confirmed in $35 \%$ of total cases; unspecified CS gas accounted for $49 \%$ and $2 \%$ were identified as mace. Male adults represented $62 \%$ of all cases.

Conclusions-CS gas is the number one chemical inquiry made to CIRS and has gen erated much concern with A\&E staff. Problems that have arisen include:

- Unpleasant immediate effects on the casualty;

- Anxiety of the casualty;

- Concerns over any pre-existing medical conditions, such as pregnancy and eczema in the casualty;

- Secondary contamination to the emergency services, vehicles, and medical equipment;

- Secondary contamination of A\&E staff and departments;

- Delayed clinical effects in the casualty and potentially for those exposed by secondary contamination.

As a result of these concerns, about both immediate and delayed clinical effects, CIRS and NPIS (London) are currently conducting a six month study.

A review of inquiries to the National Poisons Information Service (London centre) regarding injection exposures

C M Farrow, G N Volans

National Poisons Information Service (London), Medical Toxicology Unit, Guy's and St Thomas Hospital Trust, Avonley Road, London SE14 SER

Injection is an unusual route of exposure in poisoning. Little experience of the abundance of injectable agents and the lack of relevant literature prompted a review of injection exposure inquiries to the National Poisons Information Service (NPIS) (London).

After each such inquiry a questionnaire was sent to the caller to determine the progression and outcome of the case.

Over the two year review period NPIS (London) received 341 calls for information/ advice about the injection of a multitude of agents $(0.1 \%$ of total poisons inquiries $) ; 271$ $(85 \%)$ were followed up and $136(50 \%)$ completed questionnaires were returned.

All but four cases involved adults. Overall males outnumbered females by $3: 1$. Nearly half $(49 \%)$ of all cases were the result of accidental occupational exposures, $18 \%$ were deliberate self harm, and $16 \%$ intentional abuse. The remaining adult cases were the result of other accidental exposure (including therapeutic error), or criminal assault. All paediatric cases involved therapeutic errors.

Of the accidental occupational exposures, $141(85 \%)$ involved the injection of veterinary preparations. There were 17 cases involving oil based vaccines; eight of these were surgically drained and four required debridement of necrotic tissue. There were 17 cases involving injection of Micotil (Tilmicosin). Two of these patients suffered severe toxicity, including one death.

Domestic products (for example white spirit, bleach) were most commonly involved in cases of self harm. Serious toxicity was reported in $14 \%$ of these cases. Treatments included excision, debridement, fasciotomy, grafting, and plastic surgery. Intentional abuse cases mainly involved pharmaceutical agents.

Inquiries about injection exposures are relatively few; however, $17 \%$ of the cases followed up successfully resulted in severe toxicity and/or surgical management. Early and aggressive management was shown to prevent long term sequelae. It is important that more case reports are published to improve knowl edge and management of injections and, ultimately, to reduce morbidity.

Service improvements from poisons services-results from a survey of accident and emergency departments A Campbell

National Poisons Information Service (London), Medical Toxicology Unit, Guy's and St Thomas' Hospital Trust, Avonley Road, London SE14 SER

For years the National Poisons Information Service (NPIS) (London) has reported an increasing number of telephone inquiries made to it. From April to July 1997 a survey of accident and emergency departments was carried out to determine whether this rise was related to deficiency in the service provided or due to other factors, for example revised hospital policies/procedures, medicolegal reasons, etc.

Postal questionnaires were sent to both consultants and sisters of 268 different departments. Questions about hospital policies for contacting a poison centre and for handling of poisoning cases were asked. Further comments were invited. Recipients were offered various information packages.

Altogether 201 replies were received from 173 hospitals $(64.5 \%) ; 85$ from consultants, 55 from sisters/charge nurses, and the remainder from other grades. Sixty three per cent of respondents preferred being held in a call stacking system to getting an engaged tone when the service was busy; $31 \%$ preferred to get engaged tones and try another centre. Thirty per cent of replies indicated a policy to call the NPIS for every poisoning case regardless of seriousness or availability of information in-house, many giving reasons for such policies. Over $75 \%$ of replies indicated internal policy to call specific NPIS centres, again giving reasons.

Many additional comments were made. Many indicated awareness that their policies placed unnecessary and unjustified demand on NPIS resources and offered to revise them. Comments on the current service were generally favourable - the major complaint was the occasional long waiting times for initia response. Views about other information sources were expressed-a recurrent theme was preference for "voice" services as opposed to computer ones. Reasons were the ability to ask supplementary questions, receiving responses tailored to specific cases, and receiving prompts about other problems/factors for a particular case that may not have been considered. Suggestions for useful NPIS publications, on-line services, and callback facilities were received.

\section{The recovery from severe alcohol poison-} ing: report of a pilot study

Chris Moulton, Tim Rainer

Accident and Emergency Department, Royal Bolton Hospital, Minerva Road, Farnworth, Bolton, Lancs BL4 OFR

Alcohol poisoning may cause a depression in the level of consciousness as defined by the Glasgow coma score (GCS). We recorded the clinical and biochemical features of 10 such episodes and made the following observations:

- There is no simple relationship between blood alcohol concentration, level of consciousness, or recovery time. 
Table 24 Concentration dependent blockade of peak $I_{N a}$ by $A M I$ and the antidotal effects of $A A G$ (mean $\left.(S D) ; n-3-5\right)^{\dagger}$

\begin{tabular}{lllll}
\hline Amitriptyline $(\mu M)$ & 0.1 & 0.4 & 1.6 & 6.4 \\
\hline AMI alone & $0.03(0.01)$ & $0.317(0.033)^{\star}$ & $0.733(0.250)^{\star}$ \\
AMI + AAG $62.4 \mu \mathrm{l}$ & & $-0.033(0.032)^{\star \star}$ & $0.167(0.110)^{\star \star}$ & $0.95(0.071)^{\star}$ \\
\hline
\end{tabular}

†The data are the normalised blockade of peak $\mathrm{I}_{\mathrm{Na}}$ against the control peak $\mathrm{I}_{\mathrm{Na}}$. Negative values indicate that the peak current after AAG application was greater than that of control. ${ }^{\star} \mathrm{p}<0.05$ compared with control; ${ }^{\star \star} \mathrm{p}<0.05$ compared with AMI alone.

- The blood alcohol concentration may increase in the first two hours after assessment. It then falls faster than can be explained by zero order kinetics alone.

- Clinical signs of alcohol intoxication are unreliable.

- Hypoglycaemia is not a common feature; neither is electrolyte disturbance.

- Intravenous fluids have no definite effect on recovery.

We conclude that the recovery from severe alcohol poisoning is more complicated than previously described and should be investigated further. All of our patients reached a GCS of 15 within nine hours of assessment. Failure to achieve a GCS of 15 within six to eight hours of hospital admission with a diagnosis of alcohol intoxication is thus a definite indication for computed tomography of the brain.

\section{Administration of $\alpha_{1}$-acid glycoprotein} and reduction of amitriptyline toxicity

Y-L Ma, J A Henry

Academic Department of Accident and Emergency Medicine, Imperial College School of Medicine, St Mary's Hospital, London W2 1NY

Tricyclic antidepressants are well known to cause fatal arrhythmias when taken in overdose, due to their effect on sodium channels. Amitriptyline (AMI), a widely prescribed member of this group, is over $90 \%$ bound to $\alpha_{1}$-acid glycoprotein (AAG) in plasma, and administration of this plasma protein may reduce its toxicity. This study investigated the antidotal effects of AAG on AMI induced sodium current $\left(\mathrm{I}_{\mathrm{Na}}\right)$ suppression in isolated single guinea pig ventricular myocytes using whole cell patch clamp techniques.

Guinea pig myocytes were isolated by enzymic digestion and continuously perfused at room temperature. The perfusion and patch pipette solutions were chosen in order to approximate to normal physiological conditions. Sodium currents were elicited by applying a $20 \mathrm{~ms}$ test pulse of $-40 \mathrm{mV}$ after a 200 $\mathrm{ms}$ prepulse of $-90 \mathrm{mV}$ to remove inactivation. Peak currents were measured to indicate drug effects.

AMI blocked the fast voltage gated $\mathrm{I}_{\mathrm{Na}}$ in a concentration dependent manner (table 24). The data were fitted to the equation $\mathrm{Y}=\left(\mathrm{Y}_{\max }+\right.$ $\left.\mathrm{K}_{\mathrm{d}} /[\mathrm{D}]\right)^{-1}$, and gave a value for $\mathrm{K}_{\mathrm{d}}$ (IC50) of $0.69 \mu \mathrm{M}$, which is relevant to human toxic doses (free concentration of $0.4 \mu \mathrm{M} \sim 0.8 \mu \mathrm{M}$ in plasma).

AAG has a plasma concentration in man of $0.7-1.0 \mathrm{~g} / 1$. AAG $2.5 \mathrm{~g} / 1(\sim 62.4 \mu \mathrm{M})$ alone had no significant effect on sodium channel function but significantly abolished the effects of AMI (table 24). AAG deserves further study as a potential antidote for tricyclic anti- depressant cardiotoxicity. We are grateful to Bio Products Laboratory who funded this project.

Detection of alcohol misuse using the "Paddington alcohol test", with subsequent counselling by an alcohol health worker: 1998 onwards

$\mathrm{R}$ Touquet, $\mathrm{S}$ Wright, A Brown

Accident and Emergency Department, St Mary's Hospital, Praed Street, London W2 INY

Objective-To review our detection rate, with reference to the success for later counselling, of alcohol misuse by adult accident and emergency (A\&E) attenders.

Methods-Data from our second study period of one year (Smith et al 1996, Wright et al in press) are compared with the statistics of alcohol misuse for our catchment population.

Results-More than 50000 (22.2\%) residents drink in excess of sensible levels, including $12000(6.7 \%)$ considered to be dependent. Of 53090 adult A\&E attenders, approximately two thirds (35 393) are resident within our area. We therefore would expect that 7857 $(22.21 \%)$ drink hazardously, including 2371 (6.7\%) who are dependent. However A\&E staff detected only 335 patients (average 28 per month) as misusing alcohol, $4.3 \%$ of the former and $14 \%$ of the latter number.

Comment-Our first study over two years (Green et al 1993) demonstrated that A\&E is an appropriate place for the consideration of alcohol misuse, $50 \%$ of detected and referred patients reattending for help. The one minute Paddington alcohol test was then developed and validated as a practical detection tool over the second study period, $60 \%$ of patients now reattending for subsequent counselling, resulting in $65 \%$ reducing their intake at six months. Despite this success for counselling, our detection rate is low, $\mathrm{A} \& \mathrm{E}$ staff failing to refer both dependent and hazardous drinkers to the alcohol health worker. Could this be because of staff attitudes, or because of lack of time in the peripatetic $A \& E$ environment with its current staffing levels? How do we now increase the detection and referral rate?

Brief interventions for alcohol in the emergency department: a purchaser's perspective

J M Hadfield, R Duxbury, G McMahon, D W Yates

University Department of Emergency Medicine, Clinical Sciences Building Hope Hospital, Salford M6 8HD

Introduction-As part of the policy to reduce the harm caused by alcohol in the community, the commissioners for alcohol services in Salford funded the appointment of an alcohol specialist in the emergency department at Hope Hospital. Shifting the emphasis from the already damaged drinker to patients in the earlier stages of hazardous drinking has been shown to create more opportunity for effective treatment. Work at St Mary's Hospital in London has shown that problem drinkers can be detected in emergency departments and brief interventions directed towards reducing alcohol consumption have been shown to be effective. This paper reviews how the project has been managed to ensure compliance with purchaser's objectives.

Patients and methods-An alcohol specialist was employed in the emergency department each weekday morning. Patients whose attendance was known to have been associated with alcohol abuse were given an appointment with the specialist. At interview brief counselling and advice was given and in some cases the patient referred on for further help. Performance targets were set for each stage of the assessment and treatment process in order to assess the effectiveness of the service. Results -579 patients have been referred to the specialist in the first two years. Outcomes are shown in table 25 .

Conclusions-Alcohol causes significant harm in the community but there are few opportunities for effective interventions. It has been suggested that alcohol treatment facilities could be integrated with emergency departments but there are few examples of this policy being introduced or assessed. This project has demonstrated that the intervention of an alcohol specialist within hours of an alcohol related attendance to an emergency department can result in positive action by the patient. Performance targets can be set and monitored. The model could be usefully employed elsewhere to increase the effectiveness of strategies which can reduce the harm caused by alcohol.

\section{Posters: Imaging}

The use of a dedicated extremity MRI scanner by an accident and emergency department in the assessment of the clinical scaphoid fracture

M A Higgins, H Griffiths, P W Richmond Accident and Emergency Department, Cardiff Royal Infirmary, Newport Road, Cardiff CF2 $1 S Z$

To analyse the usefulness of a dedicated extremity magnetic resonance imaging (MRI) scanner in the assessment of the clinical scaphoid fracture in an accident and emergency (A\&E) setting.

Setting - A large urban A\&E department in South Wales.

Table 25

\begin{tabular}{llll}
\hline Performance targets & Expected/year & Year 1 & Year 2 \\
\hline Patient screened by A\&E staff & 390 & 249 & 330 \\
Patient agrees to appointment with alcohol specialist & 350 & 225 & 326 \\
Patient attends assessment appointment & 200 & 165 & 208 \\
Patient demonstrates understanding of key information by verbal feedback & 120 & 157 & 351 \\
Patient takes positive action verified by follow up & 60 & 61 & 373 \\
\hline
\end{tabular}


Subjects-Patients over 18 years of age with signs of a clinical scaphoid fracture at 10 days. Methods-All patients attending the A\&E department over a four month period with clinical signs of scaphoid fracture had plain radiographs performed on the day of presentation. If normal, the wrist was immobilised in plaster. Patients underwent a second examination and, if appropriate, a second set of plain radiographs on day 10 . If clinical signs persisted and plan radiographs were normal, a dedicated extremity MRI scan was performed using a small bore magnet system constructed in the medical physics department of the University Hospital of Wales. Patients with negative scans were followed up by telephone survey or questionnaire.

Results-38 patients had dedicated extremity MRI scans performed. Twelve demonstrated abnormalities (32\%). Three (8\%) had a scaphoid fracture. Two previously unsuspected fractures, of the distal radius and the trapezoid, were detected. Thirty three patients $(86 \%)$ were diagnosed as not having a significant bony injury and further immobilisation in plaster was therefore abandoned. The estimated cost per scan was $£ 150$.

Single exposure intravenous urography in the accident and emergency department-is it worthwhile?

$\mathrm{K}$ Murali, N Nayeem

Accident and Emergency Department, Lewisham Hospital, Lewisham, London SE13 6LH

Background-Patients with colicky pain referred to the urinary tract frequently attend the accident and emergency (A\&E) department. Intravenous urography (IVU) is the gold standard for contrast imaging of the urinary tract in an acute situation. Conventional IVU involves a series of exposures. There is a potential risk from over exposure to radiation in these patients when by necessity they need to be radiologically re-evaluated repeatedly. A single exposure IVU at 20 minutes is an attempt to reduce this risk.

Aim-To evaluate the effectiveness of a single exposure IVU in $\mathrm{A} \& \mathrm{E}$ in diagnosing renal colic and to evaluate the interpretation of IVU $x$ rays by the $A \& E$ doctors and the possible effect on patient outcome.

Method-The IVU report and the patient outcome on all those patients who had a single exposure IVU in the A\&E were studied over a 15 month period (January 1997 to April 1998). Results-IVU was done 200 times on 198 patients in the $A \& E$ during the study period. Sixteen $(8.51 \%)$ patients had to be excluded because of incomplete records; 182 patients were available for the study. Seventy six (41\%) patients were referred to the urological services, $26(14 \%)$ patients were referred to a non-urological specialty with an alternate diagnosis, and $81(45 \%)$ patients were discharged home after the IVU.

Altogether $95 x$ rays reported by consultant radiologists were available for this study. Fifty one of these were reported normal while 31 were abnormal and just 13 needed further specialised investigations. The A\&E doctor and the consultant radiologist agreed on 51 out of these $95 x$ rays.

Discussion-A definitive $x$ ray endpoint, that is either abnormal or normal could be reached in as high as $86 \%$ of patients. The $x$ ray reports tally well with the inference of the A\&E doctor in the case of a normal study but there seems to be a significant disparity in those cases reported to have an abnormal study. However, this needs further clarification based on a larger sample.
Absence of complication in this study population, little interference from an unprepared bowel in $x$ ray interpretation, and the high yield rate reflects very favourably in the clinical decision making and strengthens the case for doing single shot IVU in the A\&E department.

\section{What's the "angle" on supine obliques}

Paul Curran, Mark Jenkins, Sharon Conway, Laurence Rocke

Accident and Emergency Department, Royal Victoria Hospital, Grosvenor Road, Belfast BT12

Background-A case presented to the accident and emergency department that questioned the usefulness of supine oblique $x$ rays of the cervical spine. The lower cervical spine was not seen on the cross table lateral, supine view. Obliques of the cervical spine showed normal alignment but on further imaging there was a subluxation of $\mathrm{C} 5$ on $\mathrm{C} 6$. This prompted a pilot study concerning the technique employed in performing supine obliques.

Methods-An anatomical cervical spine specimen with a subluxation was imaged between zero and 90 degrees.

Results - Subluxation was increasingly difficult to see above angles of 45 degrees. The optimum angle was 30 degrees.

Conclusions-Supine obliques taken at above 45 degrees become increasingly difficult to interpret. The bar in the trolley hinders the $x$ rays taken below 45 degrees as was the above. Our recommendations are that if supine obliques are to be employed they should be performed at 30 degrees with the film offset to avoid the trolley bar.

Neuropsychological and MRI abnormalities in individuals with mild traumatic brain injury

D L Mason, D W Yates, E Berry, D Hughes, J Thorne

Department of Emergency Medicine, Hope Hospital, Stott Lane, Salford

Mild traumatic brain injury (MTBI) is a common clinical problem, accounting for between $60 \%$ and $80 \%$ of admissions for head trauma. MTBI is often associated with deficits on tests of complex attention and working memory. Furthermore, about $20 \%$ of patients with MTBI develop deleterious secondary morbidity but evidence for the aetiology of these postconcussional sequelae remains meagre. Recent studies have suggested that shearing injuries, caused by acceleration/deceleration of the brain may be important and abnormalities have been demonstrated on magnetic resonance imaging (MRI) in around $20 \%$ of patients with MTBI. However, the relationship between these observations and the development of persistent morbidity remains unclear.

In this ongoing study, 30 patients who met the criteria for MTBI received a MRI scan and were assessed with a comprehensive neuropsychological test battery within 72 hours of their injury. Patients who performed two standard deviations or below comparable normative data were considered to have an abnormal response.

Altogether $93 \%$ of patients were found to have abnormal scores on at least one neuropsychological measure. Specifically, $57 \%$ of patients showed abnormal verbal learning (California verbal learning test) and $63 \%$ showed abnormal planning and visual memory (Rey complex figure test). Twelve patients $(40 \%)$ demonstrated MRI abnormalities within 72 hours of head trauma.
These patients were found to show significantly impaired performance on specific tests of attention (paced auditory serial addition test and elevator counting with reversal) and attentional switching (Wisconsin card sorting test). Mechanism of injury was not found to be related to performance on neuropsychological measures or the presence of MRI scan abnormalities.

These initial results suggest that MRI abnormalities may be useful in separating those patients with explicit deficits on complex measures of attention from patients with a more generalised cognitive impairment. The presence of attentional deficits may be of significance to the later development of persistent morbidity in MTBI.

Bedside emergency department ultrasound: how to introduce it

P Thompson ${ }^{\star}, \mathrm{R}$ Brown ${ }^{\star}, \mathrm{P}$ Sidhu ${ }^{\star \star}, \mathrm{E}$ Glucksman ${ }^{\star}$

${ }^{\star}$ Department of Accident and Emergency Medicine and ${ }^{\star}{ }^{\star}$ Radiology Department, King's College Hospital, London SE5 9RS

Objective - The role of ultrasound for diagnostic and therapeutic intervention is well described. Our aim is to provide data on the benefits of a monitored introduction of ultrasound to the accident and emergency (A\&E) department. There are several issues which need to be considered before introducing an additional technology within the $A \& E$ department. These include type of ultrasound machine to purchase, methods of recording scans, patient satisfaction, the effect on patient management, waiting times, and the adequate training of $A \& E$ staff in the use and interpretation of ultrasound.

We present the experience gained from the introduction of emergency physician ultrasonography with reference to the King's College Hospital experience.

Methods-A report is developed after consultation with other relevant departments. A retrospective analysis provides data which indicates the potential demand for $A \& E$ department ultrasound. The introduction of ultrasound is initially on the basis of observing and stating clinical findings without directing clinical management. This prospective controlled data confirms scanning competence. The clinical use of ultrasound remains under clinical audit and departmental review.

Results - The range of clinical applications is presented. A combination of patient satisfaction, A\&E department audit of patient outcomes, and ultrasonologist review of adequacy of scans is provided.

Conclusion-The role for ultrasound within A\&E departments remains topical. Potential clinical benefits are contrasted with training concerns, audit, and departmental politics. We have presented the experience gained at King's College Hospital A\&E department in the setting up of such a program. This has resulted in the controlled introduction of a useful clinical diagnostic tool.

Can emergency physicians evaluate ultrasounds for blunt abdominal trauma?

P Thompson*, R Brown ${ }^{\star}, \mathbf{P}$ Sidhu ${ }^{\star \star}$, E Glucksman

*Department of Accident and Emergency Medicine, ${ }^{\star}$ Radiology Department, King's College Hospital, London SE5 9RS

Objective-The aim of this study is to determine the accuracy of emergency bedside 
abdominal ultrasound as performed by emergency physicians. The assessment of blunt abdominal trauma remains an often difficul diagnostic problem. Both abdominal computed tomography and diagnostic peritonea lavage (DPL) have disadvantages. Ultrasonography is non-invasive, quick, and accurate. This study aims to determine whether ultrasonography as performed by an emergency physician is appropriate in the assessment of blunt abdominal trauma compared with other modalities.

Methods-Over a six month period, we conducted a prospective study comparing the evaluation of emergency ultrasound for blun abdominal trauma performed by emergency physicians with computed tomography, DPL, or ultrasound performed by a radiologist. Ultrasonography was performed by an emergency physician not involved in the management of the patient. This ultrasonography was performed as part of the secondary survey of the patient before $x$ rays were obtained. The findings of the ultrasound were not available for the management of the patient. Ultrasonography findings were compared with those from computed tomography and DPL together with correlation to patien outcome. Ultrasound quality assurance was maintained by regular audit performed by both the ultrasound and emergency department.

Results-Ultrasound was accurate with high specificity and sensitivity for detection of abnormal focused abdominal scans. The scans were quickly performed with immediate reporting available. Quality of scans was satisfactory with improvement noted during trial period.

Conclusion-Non-radiologists are capable of accurately performing and reporting a focused assessment of the sonographic examination of the trauma patient. We have also shown that ultrasound is a suitable modality for the initial assessment of blunt abdominal trauma. The controlled introduction of the use of ultrasound by emergency physicians will be a useful clinical adjunct within the emergency department.

Cervical spine imaging in the conscious trauma patient: a survey of accident and emergency consultants within UK and Eire

Mark G Jenkins, Paul Curran, Lawrence Rocke

Accident and Emergency Department, Belfas City Hospital, Lisburn Road, Belfast BT9 $7 A B$

Objective-To define national practice in the radiological clearing of the cervical spine in the conscious adult patient.

Methods-Questionnaire survey.

Subjects-Accident and emergency medicine consultants in charge of departments seeing more than 25000 new patients per year,

Results-191 replies were received from 243 consultants $(79 \%)$. Sixty five per cent of departments have written protocols for imaging; $7 \%$ of departments use fewer than the three standard views for clearing the cervical spine. If adequate views do not visualise the cervicothoracic junction, $89 \%$ would use swimmer's views and $12 \%$ would use supine oblique views before computed tomography.

Conclusion-The majority of departments use three standard views. Swimmer's views are the most common additional view used if the C7-T1 junction is not visualised. There are problems imaging the cervical spine in trauma and national guidelines should be set up to address this.

Has MRI a place in the management of irritable hip in children?

P White, $M$ Hendry, T Beattie, Julie Boyd

Accident and Emergency Department, Royal

Hospital for Sick Children, Sciennes Road, Edinburgh EH9 1 LF

Objective-To assess the value of magnetic resonance imaging (MRI) in the management of children with atraumatic hip pain.

Design-Prospective, descriptive study on consecutive children presenting with hip pain. Subjects-Fifty consecutive children presenting with acute atraumatic hip pain whose parents gave informed consent to participate.

Main outcome measures-(1) Practicality of scanning these children; (2) ability to detect effusion compared with ultrasound; (3) ability to detect other abnormalities.

Results-Scanning without sedation was impossible for children aged 4 years and under. As on ultrasound the MRI demonstrated effusions around the hip joint. Additional pathologies in and around the hip including muscular and marrow infections (osteomyelitis), Perthes' disease, and bony abnormalities were also demonstrated using MRI. These were not demonstrated by using ultrasound.

Conclusion-These observations indicate that MRI is a beneficial technique in diagnosing hip pain in children. Whether or not MRI should replace ultrasound requires economic evaluation and further research.

Nurses can request $x$ rays appropriately, and this saves time for patients

Michael Lindley-Jones

Accident and Emergency Department, Norfolk and Norwich Hospital, Brunswick Road, Norwich NR1 3SR

Nurse requested radiology remains controversial. In 1991 the Norfolk and Norwich Hospital established a system of nurse requested $x$ rays for minor injuries in triage. This system has recently been studied. Previous studies have looked at triage nurse requested $x$ ray systems at an early stage, but this is the first study on such a well established system.

Having undergone appropriate training triage nurses may request $x$ rays following a protocol. Limb injuries except elbows, knees and femurs, but including shoulders and clavicles may be $x$ rayed by a nurse.

All patients who had an injury that met nurse requested radiology criteria entered the study. They were randomised, at triage, to nurse or doctor requested $x$ rays. The appropriateness of $x$ ray requests and the timing through the department and were recorded.

Altogether 106 patients had nurse requested $x$ rays and 113 patients had doctor requested $x$ rays. Nurses requested $x$ rays on $70 \%$ of injuries assessed, while doctors had a request rate of $79 \%$. A positive $x$ ray finding was recorded in $54 \%$ of nurse requested $x$ rays and $52 \%$ of doctor requested $x$ rays. For triage category 4 patients $(90 \%$ of patients in the study) a mean time saving of 42 minutes from triage to establishment of diagnosis was recorded. Further analysis continues.

These findings demonstrate that nurses request $x$ rays appropriately, and that time savings can be achieved for patients. Comparison with a previous study in this departmen shows that the system has improved with time. I feel that these results are due to well though out protocols, good training, and experience accumulated over seven years.

Ultrasonic evaluation of optimum positioning for lumbar puncture

William Shestak, Carl Hsu, Jason Nau, Marcelo Sandoval

Beth Israel Medical Center, NY, USA

Objectives-This study qualitatively evaluates patient preference, and quantitatively assesses changes in an intervertebral space using ultrasound as a patient assumes different positions in preparation for lumbar puncture.

Methods-This is an experimental, patien blinded convenience sample and includes al residents or nurses available within the department when the investigators were present. Ultrasound is used to evaluate the L4-L5 intervertebral space as the volunteers assume three positions appropriate for lumbar puncture: seated leaning over a table, lying in a fetal position, and seated in a fetal position with feet supported. Patients are excluded from the study if the L4-L5 interspace is not adequately visualised by ultrasound or if the volunteer could not achieve a preset degree of flexed positioning standardised for all study participants. Data collected include change in intervertebral distance and preference of the volunteer while assuming each position.

Results-For this initial pilot study $(\mathrm{n}=12)$, three measurements were made in three different positions. Using descriptive statistics, the averages at each position were compared against each other and then ranked. Of the 12 individuals studied, for seven of them, sitting with feet support was the best position $(M>0.05 \mathrm{~cm})$. In five of the 12 , sitting with feet support was tied for best $(M>0.0 \mathrm{~cm}$ and $M<0.05 \mathrm{~cm}$ ). All participants surveyed found sitting with feet support to be most preferable. Conclusions-Preliminary data suggest that a seated fetal position with feet supported increases the L4-L5 intervertebral space and patient comfort, as compared with other positions.

The utility of cranial computed tomography in patients with altered mental status and metabolic abnormalities

Rana Sherwany, Anil Mahajan, Malissa Clarke

Howard University Hospital, Washington, DC, USA

Objective-To evaluate the yield of cranial computed tomography in evaluation of atraumatic patients with altered mental status (AMS) and metabolic abnormalities without focal neurological findings.

Setting-Emergency department of an urban teaching hospital, census 37000 adult patients per year.

Data collection-A observational retrospective chart review was conducted for adult patients seen over a six month period in 1996. Charts were reviewed for all patients who presented with AMS and one or more of the following abnormalities: hypo/hypernatraemia, hypo/ hyperkalaemia, hypo/hyperglycaemia, and alcohol intoxication. Those patients with history or signs of trauma or focal neurological deficit were excluded. Clinically significant computed tomography was defined as acute stroke, acute hydrocephalus, malignancy, bleeding, or infection. 
Results - 56 patients fit the inclusion criteria. Of the $22 / 56$ patients (39.3\%) who had computed tomography, $21 / 22(95.5 \%)$ had no abnormal finding. The one patient $(4.5 \%)$ who had positive computed tomography had alcohol intoxication and subarachnoid bleed. Conclusion - With the exception of the alcohol intoxicated patient abnormal computed tomography was uncommon in these patients, suggesting that emergent computed tomography is not necessary in patients with AMS, metabolic abnormalities, no signs or history of trauma, and no focal deficit. A prospective study employing these clinical guidelines should be undertaken.

Evaluation of bowel and mesenteric injury with emergency abdominal ultrasound

John R Richards, John P McGahan, Jamie L Simpson, Payam Tabar

University of California, David Medical Center, USA

Purpose-To assess the utility of emergency ultrasound for the detection of acute bowel and mesenteric injury (BMI) in patients with blunt abdominal trauma.

Materials and methods-Prospective data of all patients who had emergency ultrasound were recorded on a data sheet with final physician interpretation. Patients with BMI were identified retrospectively. Physical examination, laboratory, computed tomography, and intraoperative findings were compared with the prospective data sheets.

Results-From $1 / 95$ to $1 / 981686$ emergency ultrasounds were performed, and 71 patients had BMI. There were 34 small bowel injuries and 53 large bowel injuries. Seventeen patients had both small and large bowel injury. There were 41 true positive ultrasounds for free fluid $(58 \%)$, and 30 false negative scans $(42 \%)$. Twenty five of the 41 patients with positive ultrasound had concomitant injuries to liver, spleen, pancreas, gall bladder, kidney, and/or bladder. The remaining 16 patients had isolated BMI with positive ultrasound, and all had damage to bowel and mesentery identified intraoperatively. All patients with false negative ultrasound except one (29/30) had abdominal tenderness. Sixteen patients with false negative ultrasound had BMI detected $\geqslant 12$ hours after the initial scan. Conclusion-BMI is frequently associated with injury to other organs, which may account for free fluid. Patients with isolated BMI uncommonly have free fluid. For clinical suspicion of BMI, observation, serial physical examinations of the abdomen, computed tomgraphy, or repeat ultrasound may be helpful in confirming the diagnosis.

\section{Posters: Therapeutics}

Is there a role for acupuncture in the emergency department management of pain?

P Thompson, R Brown, E Glucksman

Department of Accident and Emergency Medicine, King's College Hospital, London SE5 9RS

Objective-The aim of this study was to determine (1) the adequacy of pain relief obtained after soft tissue injuries; (2) the acceptance of complementary methods of analgesia within a central London teaching hospital accident and emergency (A\&E) department; (3) the appropriateness of acupuncture in the management of ankle sprains.
Methods-A telephone survey was performed on 100 patient who presented to the A\&E department with an ankle sprain over a three week period. These patients had all received sole management through the A\&E department. They were evaluated with regard to satisfaction with their pain management. They were also questioned regarding their understanding of complementary therapies available within the community and whether they would consider using acupuncture if available. Over a three month period we conducted a prospective randomised clinical trial comparing acupuncture, "sham" acupuncture, and a control group in the management of ankle sprains. All groups received oral analgesia and a support bandage together with advice regarding progressive resumption of activities. Final follow up was at six weeks.

Results-29/85 (34\%) felt their pain was not adequately controlled by the oral analgesia and would consider acupuncture to assist in the control of pain. Acupuncture was successful in alleviating pain and swelling and was well received by the patient population.

Conclusion-The control of pain remains a multifactorial problem. Acupuncture can assist in the management of a selected group of patients as part of an overall management plan. Its use for other soft tissue injuries and emergency presentations could be considered in an attempt to improve pain management.

Adrenaline upregulates the expression of $L-$ selectin on monocytes in vitro

T H Rainer, N Lam, R A Cocks

Accident and Emergency Medicine Academic Unit, Chinese University of Hong Kong and the Prince of Wales Hospital, Shatin, Hong Kong

Background-Surface adhesion molecules are essential factors in cell-cell communication and mediate leucocyte-endothelial interaction before migration at sites of inflammation. L-selectin is expressed on almost all leucocyte types and is important for initial adhesion, slowing, and rolling of leucocytes on endothelium. Little is known of the factors controlling the expression of L-selectin in the circulation prior to adhesion. After injury there is an early adrenosympathetic response which precedes increases in the expression of leucocyte L-selectin. This study investigates whether adrenaline increases the expression of L-selectin on monocytes, neutrophils, and lymphocytes in vitro and whether this is due to $\beta$ adrenergic effects.

Methods $-20 \mathrm{ml}$ of blood was withdrawn from 28 healthy volunteers ( 21 males) with a mean age of 29 years (range 23-67 years). Study 1: adrenaline at physiological doses mimicking trauma $(2.5-200 \mathrm{nmol} / \mathrm{l})$ was added to whole blood and incubated for 15 minutes. Study 2 : propranolol $\left(50 \mu \mathrm{l}\right.$ of $4 \times 10^{-4}$ was also added to separate tubes before incubation with adrenaline. Samples were analysed by flow cytometry. Saline $(40 \mu \mathrm{l} 0.9 \%$ solution) was used as a control. Expression is described as percentage of cells expressing L-selectin and average intensity (mean channel fluorescence, mcf) for individual cell types. The paired $t$ test was used to analyse percentage differences between doses of adrenaline and control. Spearman's rank correlation was used to analyse the dose effect.

Results-Significant increases in the expression of L-selectin was evident in monocytes at all doses of adrenaline $(p<0.001)$. More cells expressed L-selectin $(10 \%$ increases at adrenaline $100 \mathrm{nmol} / 1 ; t$ value $5.8 ; \mathrm{p}<0.0001$ ) and average expression also increased $(25 \%$ increase at $100 \mathrm{nmol} / 1 ; t$ value $5.7 ; \mathrm{p}<0.0001)$. Percentage cells expressing and mcf of L-selectin correlated with adrenaline dose $(Z$ value $2.19, p<0.05$ and $Z$ value $2.42 ; p<0.02$ respectively). This increase was blocked by propranolol (mean difference $24 \%$ at adrenaline $100 \mathrm{nmol} / \mathrm{l} ; t$ value $5.2 ; \mathrm{p}<0.0001)$. No significant differences were observed for neutrophils or lymphocytes.

Conclusions-Adrenaline upregulates the surface expression of L-selectin on monocytes. This effect is partially mediated by $\beta$ adrenoceptors.

Investigating health effects of herbal and traditional medicines

Debbie Shaw, Christine Leon, Virginia Murray, Glyn Volans

Medical Toxicology Unit, Guy's and St Thomas' Hospital Trust, Avonley Road, London SE14 SER

Objective-The Medical Toxicology Unit provides information to accident and emergency departments on health effects of traditional or herbal medicines which may present unexpected difficulties in patient treatment or investigation.

Background-Although most herbal medicines are relatively safe there is still the potential for these to cause adverse health effects and cases have been reported where herbal medicines have been misused or potentially toxic material has been ingested. These remedies are available in a wide range of forms, from the commercial products in supermarkets or health food stores to the specific combination of tinctures or crude herbs provided by herbal practitioners. There has been considerable growth of interest in the use of traditional medicines from China or the Indian subcontinent. Investigation of these cases is particularly complex as unusual medicinal material, of plant, animal or mineral origin, may be used.

Method-In response to case inquiries from medical professionals, relevant literature and previous case reports are reviewed. Plant material may be identified. Appropriate treatment can be decided. The findings are discussed with the reporting doctor to assess causal relationship.

Results-Annually over 1000 emergency inquiries relating to these products are received by the National Poisons Information Service (London). These include overdose, accidental ingestion, and adverse reaction reports. In a five year period (1991-95) association between symptom and product was confirmed in 12 cases, and assessed as possible/probable in 773 cases. These remedies are relatively safe when compared with western medicine, however there are some areas of concern.

Comment-We can provide information and assist in identification of herbal material, in order to help medical professionals with the assessment of cases involving the use of herbal or traditional medicines. The Traditional Medicines project can be contacted on +44 (0) 1717715380 .

Antibiotic prescribing patterns and outcome in an emergency department

Robert W Derlet, Alicia M Ledesma

University of California Davis, USA

Objectives-To determine the most common antibiotics prescribed for patients discharged from the emergency department, and to determine patient outcome. 
Methods - Charts of all patients discharged from an academic emergency department were reviewed immediately after discharge during a two month period in 1998. Patients identified as having received a prescription antibiotic at discharge were identified. The medical records were reviewed for information on treatment, age, laboratory studies, $x$ rays, and final diagnoses. Follow up calls were made to patients within 72-96 hours of the emergency department visit. Patients were asked about the clinical course of their infection and for information on any side effects.

Results-A total of 660 patients were studied, and $84 \%$ received generic antibiotics, and $16 \%$ newer brand name antibiotics. The most common antibiotics for each specific infection were as follows: bronchitis, trimethoprimsulfamethoxazole (tmx-smx) $50.3 \%$, erythromycin $19.2 \%$, amoxicillin $9.6 \%$; otitis media in children: amoxicillin $69.0 \%$, ery-sulfa $15.2 \%$; otitis media in adults: tmx-smx $41.7 \%$, amoxicillin $22.2 \%$; outpatient pneumonia: erythromycin $35.5 \%$, azithromycin $20.0 \%$; pyelonephritis: cephalexin $60.7 \%$, tmx-smx 14.3\%; sinusitis: tmx-smx $65.7 \%$, amoxicillin $20.0 \%$; urinary tract infection: tmx-smx $37.1 \%$, cephalexin $36.0 \%$. A total of 277 patients were contacted by telephone and $79 \%$ stated that symptoms of their original infection had improved. Most treatment failures occurred with bronchitis or otitis media and occurred more frequently with use of generic antibiotics when compared with brand name, but this did not reach statistical significance. Side effects were most commonly seen with use of erythromycin, tmx-smx, and amoxicillin. Side effects occurred in $52 \%$ of those patients prescribed erythromycin and consisted of abdominal pain or vomiting; $35 \%$ of these patients did not finish their course of antibiotics.

Conclusion-Most antibiotics prescribed were generic. Clinical outcome in this group of patients was overall very good, however, significant side effects were seen with erythromycin.

Patients who report an allergy to penicillin: how many truly are?

Phillip R Salen, Quan Nguyen, Ngoc Tran$\mathrm{Vu}$, Robert E O'Connor

Department of Emergency Medicine, Medical Center of Delaware, Christiana Care Health System, Newark, Delaware, USA

Objective-Allergy to penicillin is a frequent historical finding which may limit antibiotic choice. We conducted this study to determine the frequency of true penicillin allergy, explore the role of penicillin skin testing, and see how antibiotic selection could be changed after a negative test.

Methods-This prospective study was conducted at a tertiary care hospital from 9/97 to $12 / 97$. Eligibility criteria included age over 18 , reported penicillin allergy, hospitalisation, and need for parenteral antibiotics. Patients, who were critically ill, taking antihistamines, had a history of anaphylaxis, or were unable to give informed consent were excluded. Those enrolled underwent penicillin skin testing with normal saline and histamine serving as negative and positive controls. Patients were excluded if they inappropriately reacted to negative and positive controls. A positive reaction was defined by a wheel and flare reaction to Pre-Pen or penicillin G. Statistical analysis was performed using the $95 \%$ confidence interval (CI).
Results - Of the 31 subjects screened, 26 were enrolled. Positive skin testing was observed two patients $(7.6 \%$; CI $1.3 \%$ to $21.6 \%)$. There were no adverse reactions to penicillin skin testing. Of the 24 patients with negative tests, $18(75 \%$; CI $55 \%$ to $89 \%)$ were switched to a preferred $\beta$ lactam antibiotic. None of 18 subjects given a $\beta$ lactam antibiotic after negative skin testing suffered an adverse event.

Conclusions-True penicillin allergy is uncommon among subjects with a history of atypical reactions. Penicillin skin testing appears safe and accurate when used to determine allergy status. Negative testing led to changes in antibiotic choice with increased $\beta$ lactam antibiotic use. Patients who report allergy to penicillin characterised by atypical reactions should undergo skin testing, especially in circumstances where a $\beta$ lactam antibiotic offers significant advantage over alternate agents.

\section{Posters: Emergency Department} Administration

Using business modelling software to design and operate clinical service systems

David R Eitel, Christopher Brown, Linda Horner, Sharon Pitzer, Michael Kleinman, Tom Cyr, Richard Wuerz

York Hospital and Brigham and Women's Hospital, $P A, U S A$

Background - Health delivery systems everywhere are under increasing pressures to improve both efficiency and effectiveness of care delivery. Manufacturing and other service industries have turned toward a process way of thinking, using a variety of process oriented tools, for similar purposes. Business modelling software is one such tool now available in affordable, PC based formats, and used increasingly in non-health industries to predictively design and manage operations.

Hypothesis - A group of health industry professionals could use business modelling software to predictively design and operate a new off-campus urgent care center (UCC) in the United States.

Methods-Incorporating previously applied work from the areas of strategic marketing, operations management, activities based cost cutting, and performance measurement, expected market segments were first identified within the geographic setting of the planned UCC. Ten service offerings were then described within the context of its planned diagnostic capabilities. Flow charts representing the 10 service offerings were developed and loaded into the modelling software. Operational logic was then added to the scenario. Results-Simulations completed in the mode were able to: (1) identify responses of the assigned resources to varying service demands, (2) identify constraints to service throughput and quantitatively evaluate alternative solutions, (3) measure service capacity of the UCC under varying demand mixes, (4) assess the impact of technology insertion, (5) highlight the necessary metrics for the demand tracking and performance measurement systems of the UCC, (6) cost the service delivery processes in terms of resources consumed (operational costing), (7) quantify the amount of time patients spent waiting for operations and services, and (8) visually display the performance of the UCC.
Conclusions-Health industry professionals should find business modelling and simulation software tools strategically and operationally useful in the design and operation of clinical service systems.

Physician's personal conflict resolution tactics and attitudes towards domestic violence

Vicken Y Totten, David B Sugarman

Catholic Medical Center of Brooklyn and Queens, $N Y$, and Rhode Island College, USA

Introduction-Physicians' personal conflict resolution strategies may reflect their experiences of domestic violence.

Objectives-(1) Pilot a survey instrument for a larger study; (2) describe emergency physician experiences in domestic conflict resolution; (3) describe emergency physician attitudes about domestic violence.

Population-Non-representative sample of 26 physicians: nine female and 17 male, representing 18 states, at 1997 Scientific Assembly. The average respondent was 47 years old, married, and white.

Method-A self administered survey assessed emergency physician attitudes and experiences of conflict resolution, with asking and reporting domestic violence, and asked about items associated with lethal domestic violence. Results - Three physicians had been abused, two had seen a health care worker or counsellor because of domestic violence. One would be offended if asked about abuse. Two (7.7\%) had injured a partner with in the last year seven $(27 \%)$ had been physically assaulted by a partner, and four $(15.4 \%)$ had been injured by a partner within the last year. Twelve had witnessed domestic violence in a nonprofessional setting, and eight kept a gun Three $(11.5 \%)$ had coerced a partner and three $(11.5 \%)$ had been coerced into unwanted sexual acts. Nineteen $(73.1 \%)$ had been psychologically aggressive towards a partner and $84.6 \%$ had been the recipient of psychological aggression.

Conclusions-(1) A significant proportion of these emergency physicians have experienced partner assault. (2) A large majority of these emergency physicians experienced psychological aggression. (3) Emergency physicians may be hesitant about investigating potential domestic violence because of their personal experiences.

Expectations of patients arriving in an emergency department

Robert W Derlet, Alicia M Ledesma

University of California Davis, USA

Objective-To determine patient expectations for care in the emergency department and preferred qualifications of physicians providing emergency department care.

Methods-A random survey of patients over two weeks was performed in an academic emergency department waiting room. The survey asked patients to rank in order of importance eight items with \#1 the most important and \#8 the least important. These included: (1) appealing and clean waiting room, (2) waiting time to see the doctor, (3) time the doctor spends with the patient, (4) politeness of the doctors, (5) time the nurse spends with you, (6) relief of pain patients may have, (7) correct and accurate diagnosis, and (8) explanation of diagnosis and treatment. Mean preferences were calculated by dividing the total sum of preferences by the total number of completed surveys. Patients 
numbers of patients and admissions, led in different resource categories.

\begin{tabular}{llll}
\hline Condition & Public (\% EMC) & HCW $(\%$ EMC) & Significance $\left(\chi^{2}\right)$ \\
\hline Back pain, unable to walk & 57 & 95 & $<0.001$ \\
Severe headache & 57 & 91 & $<0.001$ \\
Uncontrolled bleeding & 79 & 98 & $<0.001$ \\
Possible broken bone & 68 & 92 & $<0.001$ \\
Difficulty breathing & 77 & 98 & $<0.001$ \\
\hline
\end{tabular}

HWC=health care worker.

were also asked to select from several choices the type of physician they preferred.

Results-A total of 261 patients completed questionnaires. Mean patient preferences from most important to least important were: (1) waiting time $3.63,(2)$ correct and accurate diagnosis 3.65, (3) relief of pain 3.87, (4) explanation and diagnosis of treatment 4.13 (5) patient-doctor time 4.35, (6) politeness of doctors 4.82 , (7) time the nurse spends with the patient 5.34, and (8) clean waiting room 5.87. Preferences for physicians were as follows: board certified in emergency medicine $44 \%$; does not matter what type of physician $25 \%$; internist $10 \%$; family practitioner $7 \%$; surgeon $6 \%$; non-board certified emergency physician $5 \%$; paediatrician $2 \%$; obstetrician/gynaecologist $2 \%$.

Conclusion-Patients prefer to see board certified emergency physicians in emergency departments. The most important aspects of their visit include correct diagnosis, relief of pain, and waiting time.

What chief complaints need emergency department care? Layperson $v$ health providers

Robert W Derlet, Alicia M Ledesma

University of California Davis, USA

Objective-To compare opinions between the general public and health care workers on which medical conditions require emergency department care.

Methods-A survey of a random sample of persons at malls, on the street, and of health care workers at hospitals was conducted July to December 1997 in California. Persons over age 18 were asked to complete a survey form. This form asks persons to determine which of 30 chief complaints they think requires care in an emergency department. Demographic information was also collected. Health care worker was defined as MD, PA, NP, or RN. Results-A total of 1018 non-health care and 126 health care workers completed survey sheets at multiple locations. Two potentially serious conditions were perceived as requiring emergency department care similarly between the public and health workers: severe abdominal pain $(94 \% v 99 \%)$ and severe chest pain ( $96 \% v 99 \%)$. Conditions for which there was disagreement are shown in table 26.

No significant difference in opinions on which conditions needed emergency department care was seen between groups for some minor conditions: (public $v$ health care worker): mild headache $(7 \%$ v $10 \%)$, sore throat $(11 \%$ v 13\%), cough (11\% v $11 \%)$, flu symptoms (12\% v 9\%), and minor foot prob- lems ( $4 \% v 5 \%)$. No significant difference in answers occurred when age groups, occupations, or locations were compared.

Conclusion-Laypersons tend to be conservative in their perception of what conditions need care in emergency departments, including several potentially serious problems. Both groups agree that many perceived minor medical complaints do not require emergency department care.

A resource based acuity scale for emergency medicine

Richard C Wuerz, David R Eitel

Brigham and Women's Hospital and Harvard

Objectives-Current emergency department triage emphasises how long the patient can wait before full evaluation, but does not predict resource utilisation or disposition time once the patient is seen. This study is a computer model of the performance of a new resource based acuity scale (RBAS).

Methods-A five level acuity scale with examples of presenting problems and an algorithm Each RBAS level includes probabilities of use for 11 emergency department resources (mechanical ventilation, blood transfusion, 1:1 nursing, monitor, laboratories/ electrocardiography, radiography, special tion, admission/transfer, and RBRVS code), and an expected time to disposition ranging from 30 minutes (level 5) to six hours (level 3 ). The model was run for three hypothetical hospitals with an annual census of 40000 patients with $25 \%$ admission rate, but with different RBAS casemix distributions. Hospital $A$ is an academic referral centre, hospital $B$ is a private community hospital, and hospital C is a public hospital serving a medically underserved area.

Results-The RBAS model predicted variation in resource use by as much as $118 \%$ (mechanical ventilation, hospital $\mathrm{C}>\mathrm{B}>\mathrm{A}$ ) The least variable resource was special studies $(16 \%$, hospital $B>C>A)$. Summation of disposition times varied $87 \%(B>A>C)$, and RBRVS level 5 revenue varied $24 \%$ (C>B $>A$ ). Each hospital, though processing the same

Table 27

\begin{tabular}{llll}
\hline & Appropriate & Inappropriate & Missed \\
\hline Hospital staff & $54(5)$ & $9(1)$ & $974(94)$ \\
Pharmacy database & $1015(98)$ & $22(2)$ & $\star$ \\
\hline
\end{tabular}

$p<0.00001$. Values are number $(\%)$ gations, it is common to use staff members Medical School, USA for rapid clinical assessment is described studies, parenteral medicines/fluids, consultaferences in resource utilisation among three emergency departments with identical visits and admissions. The implications for emergency department operations, throughput time, and finances will be described. The RBAS is presently undergoing prospective validation.

The use of automation to identify eligible patients during clinical investigation: how applicable is this to emergency medicine?

Phillip R Salen, Robert E O'Connor

Department of Emergency Medicine, Medical Center of Delaware, Christiana Care Health System, Newark, Delaware, USA

Objectives-When conducting clinical investiwith simultaneous clinical responsibilities to identify potential study subjects. This strategy may contribute to selection bias through convenience or judgmental sampling. We conducted this study to compare two methods of subject identification.

Methods-This prospective study was conducted at a tertiary care hospital from $9 / 97$ to $12 / 97$. Before initiation of the study, the hospital staff received training on eligibility criteria through multiple media. Additionally, a pharmacy database was created to identify potential subjects and notify the investigators daily by e-mail. Eligibility criteria included age over 18 , reported penicillin allergy, hospitalisation, and need for parenteral antibiotics. Patients, who were critically ill, taking antihistamines, had a history of anaphylaxis, or were unable to give informed consent were excluded. All admitted patients were eligible. Subjects identified by either method were classified as appropriate or inappropriate based on inclusion criteria. Subjects not identified were denoted as missed. Statistical analysis was performed using $\chi^{2}$.

Results-A total of 1037 subjects were screened; see table 27.

The pharmacy database was able to identify a higher proportion of appropriate patients $(1015 / 1037 ; 98 \%)$ than hospital staff $(54 / 63$; $86 \% ; \mathrm{p}<0.0001)$.

Conclusion-Use of an automated system facilitates reliable identification of study subjects. Use of a emergency department staff based referral system misses the majority of subjects and may introduce selection bias. Automation should be used whenever possible when conducting clinical investigation in emergency medicine.
Conclusions-This RBAS predicted large dif- 